\title{
A Magnetic Sensor to Measure Wear in Centrifugal Pumps
}

\author{
by \\ Ramin Khoie \\ BASc, The University of British Columbia, 2013
}

\begin{abstract}
A THESIS SUBMITTED IN PARTIAL FULFILLMENT
OF THE REQUIREMENTS FOR THE DEGREE OF
\end{abstract}

Master of Applied Science

in

THE FACULTY OF GRADUATE AND POSTDOCTORAL STUDIES

(Mechanical Engineering)

The University of British Columbia

(Vancouver)

April 2016

(C) Ramin Khoie, 2016 


\section{Abstract}

On average, centrifugal pumps consume between $25 \%$ and $60 \%$ of the total consumed electrical energy inside process plants. Erosion inside open-impeller centrifugal pumps leads to a reduction in pump efficiency and occasional plant downtime. This work demonstrates a new concept for an online instrument capable of monitoring wear with the objective of improving the maintenance scheduling of centrifugal pumps and the prevention of unexpected failure through a predictive maintenance system. A magnetic wear sensor is designed and fabricated that allows for wear measurement while the pump is in operation. This sensor can be installed on existing centrifugal pumps and does not require any pump modifications. Wear mostly occurs on the tip of the impeller blades reducing the thickness of the impeller which in turn increases the gap width between the impeller and the side plate inside the pump housing, from $0.65 \mathrm{~mm}$ (no wear) to $2.50 \mathrm{~mm}$ for maximum allowable wear on the pump used for prototyping. By using a magnetic circuit with the pump and its components, wear is estimated by measuring the change in the width of the varying gap between the impeller and the side plate. To assemble the magnetic circuit, a high-permeability clamping mechanism with a relative permeability of 10,000 is designed and fabricated along with a magnetic coil excited using a $1.0 \mathrm{~V} \mathrm{AC}$ voltage signal at $70 \mathrm{~Hz}$ to drive flux through the circuit. As wear occurs, the total reluctance of the magnetic circuit increases causing the inductance of the coil to drop. The coil's inductance is also a function of the impeller's angular position. To estimate wear, data is collected at a sampling frequency of $500 \mathrm{kHz}$ and then assessed in the frequency domain after fast Fourier transform (FFT). The amplitude of the FFT signal at the frequency correlated with the pump's rotational speed is then considered to estimate wear. For a data sampling time of one second the sensor has a signal to noise ratio of $17.8 \mathrm{~dB}$ with an average sensitivity of $0.022 \mathrm{mV} / \mathrm{mm}$ and a resolution of $0.38 \mathrm{~mm}$. 


\section{Preface}

The work in Chapter 2 has been presented at the IEEE Sensors 2015 conference in Busan, South Korea and the conference paper has been published in the IEEE Xplore database. (R. Khoie, B. Gopaluni, J. A. Olson, and B. Stoeber, A magnetic sensor to measure wear in centrifugal pumps, in SENSORS, 2015 IEEE, Nov 2015, pp. 14. [1])

All of the experimental work presented in Chapter 3 are conducted in a pilot plant test facility at the Pulp and Paper Center at the University of British Columbia.

This work was performed under the supervision of Dr. Boris Stoeber and Dr. Bhushan Gopaluni. The project is a part of Energy Reduction in Mechanical Pulping research program led by Dr. James Olson that is a collaboration project between mechanical pulp producers, associated supplier industries, research institutes, universities, utilities and governmental bodies. 


\section{Table of Contents}

Abstract .......................... ii

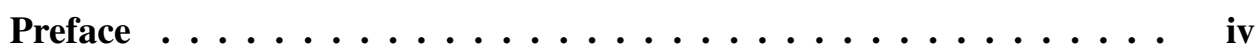

Table of Contents $\ldots \ldots \ldots \ldots \ldots \ldots$

List of Tables $\ldots \ldots \ldots \ldots \ldots \ldots \ldots$ viii

List of Figures $\ldots \ldots \ldots \ldots \ldots \ldots \ldots \ldots \ldots$

Nomenclature $\ldots \ldots \ldots \ldots \ldots \ldots \ldots \ldots \ldots$

Acknowledgments ................... xix

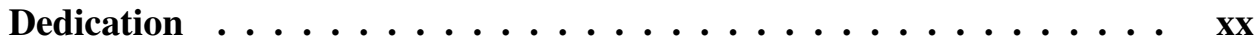

1 Introduction ........................ 1

1.1 Motivation .................... 1

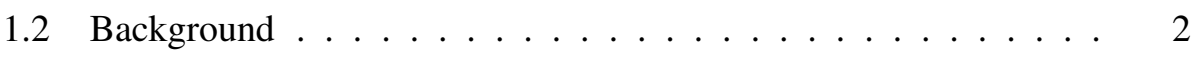

1.3 Objectives .................... 6

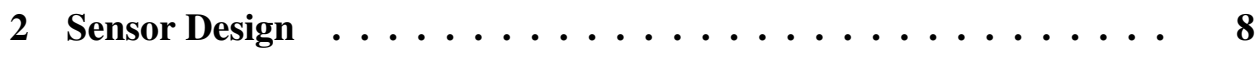

2.1 Sensor Principle . . . . . . . . . . . . . . . . . . . . . 8

2.2 Sensing Method Variation \& Comparison . . . . . . . . . . 15

2.2.1 Permanent Magnet Model . . . . . . . . . . . . 15

2.2.2 Electromagnet Model . . . . . . . . . . . . . . 21 
2.2.3 Coil Inductance Model . . . . . . . . . . . . . . . 25

2.2.4 Method Selection . . . . . . . . . . . . . 27

2.3 Flux Guide Design . . . . . . . . . . . . . . . . . . 30

2.3.1 Material Selection . . . . . . . . . . . 30

2.3.2 Sensor Geometry . . . . . . . . . . . . . . . 33

2.3.3 Numerical Validation . . . . . . . . . . . . . . 37

2.4 Sensor Instrumentation $\ldots \ldots \ldots$

2.4 .1 Coil Design . . . . . . . . . . . . . . . . . . 40

2.4 .2 Inductance Measurement . . . . . . . . . . . . . 44

2.4 .3 Circuit Design . . . . . . . . . . . . . 48

2.5 Data Analysis . . . . . . . . . . . . . . . . . . . . . 49

2.5.1 Expected Sensor Response . . . . . . . . . . . . . 50

2.5.2 Data Analysis Method . . . . . . . . . . . 53

3 Experimental Validation . . . . . . . . . . . . . . . 54

3.1 Sensor Prototype . . . . . . . . . . . . . . . . 54

3.1 .1 Flux Guide Assembly . . . . . . . . . . . . . . 54 54

3.1 .2 Magnetic Coil . . . . . . . . . . . . . 56

3.1.3 Instrumentation Box $\ldots \ldots \ldots \ldots$

3.2 Sensor Characterization . . . . . . . . . . . . . . . . . . . . 59

3.2.1 Coil Inductance and Parasitic Capacitance . . . . . . . . . 60

3.2.2 Circuit Optimization . . . . . . . . . . . . 63

3.2.3 Benchtop Experiments . . . . . . . . . . . . 64

3.3 Experimental Setup . . . . . . . . . . . . . . . . . . . 66

3.3 .1 Pump Loop . . . . . . . . . . . . . . . . 66

3.3.2 Pump Adjustments . . . . . . . . . . . . . . 67

3.3.3 Sensor Installation $\ldots \ldots \ldots 8$

3.3.4 Sensor Calibration . . . . . . . . . . . . . . . . . . 69

3.4 Experimental Results \& Analysis . . . . . . . . . . . . . . 71

3.4 .1 Sensor Output $\ldots \ldots \ldots \ldots$. . . . . . . . . . .

3.4.2 Pump Rotational Speed Variation . . . . . . . . . . 72

3.4.3 Signal to Noise Ratio (SNR) . . . . . . . . . . . 73

3.4.4 Sampling Optimization . . . . . . . . . . . . 74 
3.4.5 Data Collection . . . . . . . . . . . 75

3.4 .6 Wear Detection . . . . . . . . . . . . . . 79

4 Conclusions and Future Work $\ldots \ldots \ldots$

4.1 Conclusions . . . . . . . . . . . . . . . . 83

4.2 Future Work . . . . . . . . . . . . . . . . . . . . . . 84

Bibliography $\ldots \ldots \ldots \ldots \ldots \ldots \ldots$

Appendix A Computer Simulations . . . . . . . . . . . . . 90

A.1 Simplified Model . . . . . . . . . . . . . . . . . . 90

A.2 Advanced Model . . . . . . . . . . . . . . . . . . 92

Appendix B MATLAB Code . . . . . . . . . . . . . . . . 9 94

B.1 FFT Calculation . . . . . . . . . . . . . . . . . . . . . 94

B.2 Signal Denoising and SNR Calculation . . . . . . . . . . . 98

B.3 Scatter Plot Generation and Calibration Curve . . . . . . . . . . . 101

Appendix C Sensor Optimization . . . . . . . . . . . . . 105

C.1 Permanent Magnet Geometry Optimization . . . . . . . . . 105

C.2 Signal Quality Improvement ． . . . . . . . . . . . . . . 106 


\section{List of Tables}

Table 2.1 Physical dimensions of the pump components. . . . . . . . . . 13

Table 2.2 Effective cross-sectional areas of the pump components. . . . . 13

Table 2.3 Magnet's physical parameters. . . . . . . . . . . . . . 19

Table 2.4 Magnetic coil's physical parameters. . . . . . . . . . . 23

Table 2.5 The comparison of the three sensing methods. . . . . . . . 30

Table 2.6 High-permeability magnetic materials for the fabrication of the flux guide assembly. . . . . . . . . . . . . . 32

Table 2.7 Properties of the American Wire Gape AWG30. . . . . . . . . 41

Table 2.8 Parameters for the parasitic capacitance analytical equation. . . 43

Table 3.1 Cost of the material and the components used for the fabrication of the physical prototype. . . . . . . . . . . . . 59 


\section{List of Figures}

Figure 1.1 Cross-sectional diagram of a typical two blade open-impeller centrifugal pump. . . . . . . . . . . . . .

Figure 1.2 Two-blade pump impeller. The typical location of wear on the impeller is labeled on the figure. . . . . . . . . . . 3

Figure 1.3 Top view of a two blade open impeller made from cast iron. The suitable location for wear measurement is labeled on the figure. . . . . . . . . . . . . . . .

Figure 1.4 Westcan 40-Hp two blade open impeller centrifugal pump installed in the Pulp and Paper Center pilot plant at The University of British Columbia. The motor connected to the pump is also shown in this picture. . . . . . . . . . . 7

Figure 2.1 The diagram of a simple magnetic circuit with a single gap. . 10

Figure 2.2 The schematic drawing of the magnetic circuit created with the pump and the flux guide assembly. The dotted line represents the path of the magnetic flux inside the circuit. The location of the wear on the tip of the impeller is indicated on the drawing.

Figure 2.3 Simplified model of the pump and the sensor's flux guide with each component labeled on the diagram. . . . . . . . . . 14

Figure 2.4 Magnetic hysteresis loop. . . . . . . . . . . . . . . . . . 16

Figure 2.5 Demagnetization curve of a typical rare-earth magnet superimposed on the magnet's loadline. . . . . . . . . . . . 
Figure 2.6 Plot of the operating magnetic flux density $B_{m}$ as a function of the varying gap width $l_{v g}$ as it increases from the no-wear state $(0.65 \mathrm{~mm})$ to the maximum-wear state $(2.50 \mathrm{~mm})$ for the analytical calculation using the permanent magnet model. . .

Figure 2.7 Magnitude of flux density [T] inside the sensor and the simplified pump for the analysis of the permanent magnet sensor model generated using Comsol Multiphysics. . . . . . . . .

Figure 2.8 Plot of the magnetic flux density $B_{m}$ as a function of the varying gap width $l_{v g}$ for the case of the permanent magnet model derived from Comsol Simulation. . . . . . . . . . . . .

Figure 2.9 Plot of the operating magnetic flux density $B_{m}$ as a function of the varying gap width $l_{v g}$ as it increases from the no-wear state $(0.65 \mathrm{~mm})$ to the maximum-wear state $(2.50 \mathrm{~mm})$ for the analytical calculation using the electromagnet model. . . . . .

Figure 2.10 Plot of the magnetic flux density $B_{m}$ as a function of the varying gap width $l_{v g}$ for the case of the electromagnet model derived from Comsol Simulation. . . . . . . . . . . . . .

Figure 2.11 Coil inductance as a function of the varying gap width calculated using the analytical model of the coil inductance. . . . .

Figure 2.12 The inductance of the magnetic coil as a function of the varying gap width for the case of the inductance model derived using Comsol simulation. . . . . . . . . . . . . . .

Figure 2.13 The percentage change in the measuring parameter as a function of the varying gap width for the permanent magnet model, electromagnet model, and the inductance model derived from Comsol simulations. . . . . . . . . . . . . .

Figure 2.14 The percentage change in the inductance of the coil (output signal) using various magnetic permeability values for the flux guide assembly derived from the analytical model. . . . . . .

Figure 2.15 Sensitivity comparison of alternative material compositions for the flux guide assembly using carbon steel and high-permeability M100 material derived from Comsol simulations. . . . . . . . 
Figure 2.16 Percentage change in the output signal as a function of the varying gap width using different lengths for the flux guide assembly derived from the analytical model. . . . . . . . . . .

Figure 2.17 Percentage change in the output signal as a function of the varying gap width using various cross-sectional areas for the flux guide assembly derived from the analytical model. . . . .

Figure 2.18 A 'C' Clamp design for the geometry of the flux guide assembly with the five legs numbered on the drawing. . . . . . . . 36

Figure 2.19 The isometric drawing of the flux guide clamping mechanism. $\quad 37$

Figure 2.20 The dimensions of the flux guide assembly in [mm]. . . . . . 38

Figure 2.21 Exploded view of the more realistic pump model and the sensor created in Comsol Multiphysics. All the components of the pump and the sensor are labeled on the figure. . . . . . . . . .

Figure 2.22 Path of magnetic flux inside the sensor and the flux guide assembly. . . . . . . . . . . . . . . .

Figure 2.23 Comsol simulation results yielding the magnetic coil inductance as a function of the varying gap width for the more realistic model of the sensor and the pump. The coil is excited using a constant current source of $10 \mathrm{~mA}$ at $100 \mathrm{~Hz}$. . . . . . .

Figure 2.24 The design of the bobbin for the magnetic coil to be placed on the flux guide assembly with the dimensions in [mm]. . . . . .

Figure 2.25 Simple RL circuit to measure the inductance of the magnetic coil. . . . . . . . . . . . . . . . . .

Figure 2.26 More realistic RLC circuit representing the coil connected in series with a resistor. . . . . . . . . . . . . .

Figure 2.27 The change in the frequency response of the sensor as the magnitude of the varying gap increases from $0.65 \mathrm{~mm}$ to $2.50 \mathrm{~mm}$ derived from the analytical model of the sensor. . . . . . . . .

Figure 2.28 The change in the frequency response of the sensor as the magnitude of the varying gap increases from $0.65 \mathrm{~mm}$ to $2.50 \mathrm{~mm}$ with the addition of the external capacitance $C_{e x t}$ to the circuit. The plot is generated from the analytical model of the sensor. . 
Figure 2.29 The complete drawing of the sensor's circuit including the voltage follower circuit. . . . . . . . . . . 50

Figure 2.30 Inductance of the magnetic coil as a function of the impeller's angular position for both the no-wear and the maximum-wear cases derived from Comsol simulations. . . . . . . . . . . .

Figure 2.31 Expected FFT response of the generic time signal from the sensor while the pump is operating at 900 RPM. . . . . . . . .

Figure 3.1 The epoxy used to attach the flux guide components with the sintered M100 particles acquired from National Magnetics Group. 56

Figure 3.2 The fabricated flux guide assembly with the magnetic coil. . . 57

Figure 3.3 The flux guide assembly with the cushioning foam. . . . . . 58

Figure 3.4 The design of the coil bobbin in SolidWorks. . . . . . . . . 58

Figure 3.5 Prototype of the magnetic coil. . . . . . . . . . . . . . . 59

Figure 3.6 The instrumentation box with the electrical circuit and the DAQ unit. The box also includes a pivoting door (not shown in the picture) to protect the instruments inside. . . . . . . . 60

Figure 3.7 Picture of the complete sensor prototype including the flux guide assembly, the coil, and the instrumentation box. . . . .

Figure 3.8 Frequency response of the sensor: experimental measurements and the least square estimation. . . . . . . . . . . . . . . .

Figure 3.9 Maximizing the change in the output signal as a function of the resistance value of $R$. . . . . . . . . . . . . . . .

Figure 3.10 Maximizing the change in the output signal as a function of the excitation frequency $f \ldots \ldots \ldots \ldots$

Figure 3.11 The benchtop experimental apparatus with the sensor placed perpendicularly to the two parallel cast iron plates. . . . . . .

Figure 3.12 The frequency response of the sensor as the gap between the two parallel cast iron plates vary from $0.0 \mathrm{~mm}$ to $2.0 \mathrm{~mm}$. . . .

Figure 3.13 Westcan Centrifugal pump installed in the PPC Pilot Plant. Direction of the flow is labeled on the figure. . . . . . . . 66

Figure 3.14 Schematic diagram of the pump loop in the Pulp and Paper Center at UBC. . . . . . . . . . . . . . . . . . . . . 
Figure 3.15 The magnetic polymer mixture poured on the pump's side plate. 68

Figure 3.16 The sensor assembly installed on the pump. . . . . . . . . . . 69

Figure 3.17 The location of the ring gaskets on the schematic cross-sectional view of the pump. . . . . . . . . . . . 70

Figure 3.18 The location of the ring gaskets on the centrifugal pump. . . . 70

Figure 3.19 The output voltage signal from the sensor as a function of time for a) the pump rotational speed of 0 RPM, and b) the pump rotational speed of 900 RPM. . . . . . . . . . . . . . 71

Figure 3.20 The FFT plot of the sensor output while the pump is operating at 900 RPM. . . . . . . . . . . . . . . . . . .

Figure 3.21 The amplitude spectral density of the sensor output for various pump rotational speeds. . . . . . . . . . . . . . . .

Figure 3.22 The plot of the output signal and the noise signal at the impeller rotational speed of 900 RPM. Signal is sampled at a sampling frequency of $50 \mathrm{~Hz}$ for a duration of 1 second. . . . . . . . . .

Figure 3.23 Sensor output collected at different sampling frequencies of $20 \mathrm{kHz}, 50 \mathrm{kHz}, 100 \mathrm{kHz}$, and $500 \mathrm{kHz}$. The SNR value of each data is labeled on the plot. All measurements were taken during 1 second with a pump rotational speed of 900 RPM. . . 76

Figure 3.24 Signal to noise ratio in $[\mathrm{dB}]$ as a function of the sampling frequency for a one-second measurement on the pump running at 900 RPM. . . . . . . . . . . . . . . .

Figure 3.25 Signal to noise ratio in $[\mathrm{dB}]$ as a function of the sampling frequency for 1M data points. . . . . . . . . . . . . . . 77

Figure 3.26 Signal to noise ratio in $[\mathrm{dB}]$ as a function of the total number of samples with a fixed sampling frequency of $50 \mathrm{kHz}$. . . . .

Figure 3.27 Scatter plot of the sensor output signal at $30 \mathrm{~Hz}$ versus the output signal $15 \mathrm{~Hz}$ for various gap widths. Each data point refers to 1 second of measurement collected at $500 \mathrm{kHz}$ sampling frequency. There are 36 data points for each gap width. . . . . . 79 
Figure 3.28 Mean and the standard deviation of the scatter plot for the sensor output signal at $30 \mathrm{~Hz}$ versus the output signal $15 \mathrm{~Hz}$ at various gap widths. The sampling frequency is set to $500 \mathrm{kHz}$ and measurements are taken for a duration of 36 seconds. . . .

Figure 3.29 The mean amplitude of the sensor's output signal at $15 \mathrm{~Hz}$ versus the magnitude of the varying gap width. The error bar and the uncertainty of the gap width for each data point is shown on the plot. . . . . . . . . . . . . . . .

Figure 3.30 Comparison between the experimental results and the simulation results. The plot shows the percentage change in the output of the sensor as a function of the varying gap width as it increases from $0.4 \mathrm{~mm}$ to $2.65 \mathrm{~mm}$. . . . . . . . . .

Figure A.1 Magnitude of flux density [T] inside the sensor and the simplified pump for the analysis of the electromagnet sensor model generated using Comsol Multiphysics. . . . . . . . . . . .

Figure A.2 Magnitude of flux density [T] inside the sensor and the simplified pump for the analysis of the inductance sensor model generated using Comsol Multiphysics. . . . . . . . . . . . . 92

Figure A.3 Magnitude of the flux density [T] inside the flux guide assembly. The saturation flux density for M100 material is $0.42 \mathrm{~T}$. .

Figure C.1 Scatter plot of the ratio of signal at $30 \mathrm{~Hz}$ over $70 \mathrm{~Hz}$ versus the signal ratio of $15 \mathrm{~Hz}$ over $70 \mathrm{~Hz}$ at various gap widths. . . 107

Figure C.2 Scatter plot of the ratio of signal at $30 \mathrm{~Hz}$ and $15 \mathrm{~Hz}$ over the RMS value of noise. . . . . . . . . . . . . . 108

Figure C.3 Scatter plot of the denoised amplitude at $30 \mathrm{~Hz}$ versus the denoised amplitude at $15 \mathrm{~Hz}$ at various gap widths. . . . . . . 109 


\title{
Nomenclature
}

\author{
$\varepsilon_{0} \quad$ Absolute permittivity \\ $\varepsilon_{r} \quad$ Relative permittivity of wire insulation (enamel) \\ $\hat{V}_{\text {noise }}$ Signal noise in frequency domain \\ $\hat{V}_{\text {signal }}$ Denoised output signal in frequency domain \\ $\mu \quad$ Magnetic permeability \\ $\mu_{p} \quad$ Relative permeability of pump components \\ $\mu_{0} \quad$ Magnetic permeability of free space \\ $\mu_{r} \quad$ Relative permeability \\ $\omega \quad$ Angular frequency \\ $\omega_{\text {pump }}$ Rotational speed of pump impeller \\ $\Re \quad$ Reluctance \\ $\Re_{\text {const }}$ Constant reluctance \\ $\mathfrak{R}_{f g} \quad$ Reluctance of the flux guide assembly \\ $\mathfrak{R}_{\text {gap }} \quad$ Reluctance of the air gap \\ $\mathfrak{R}_{\text {var }} \quad$ Varying reluctance \\ SNR Signal to noise ratio
}


$\mathrm{SNR}_{\mathrm{dB}}$ Signal to noise ratio in decibels

$\vec{B} \quad$ Magnetic flux density

$\vec{H} \quad$ Magnetic field strength

A Area

$A_{s} \quad$ Cross-sectional area of the secondary gap

$A_{c g} \quad$ Effective area of the constant gap width

$A_{\text {coil }} \quad$ Cross-sectional area of the magnetic coil

$A_{c} \quad$ Cross-sectional area of the flux guide assembly

$A_{\text {gap }} \quad$ Cross-sectional area of the air gap

$A_{\text {im }} \quad$ Effective area of the pump impeller

$A_{\text {magnet }}$ Cross-sectional area of the permanent magnet

$A_{s p} \quad$ Effective area of the side plate

$A_{t b} \quad$ Effective area of the stuffing box

$A_{v g} \quad$ Effective area of the varying gap width

$B_{m} \quad$ Operating flux density of the permanent magnet

$B_{r} \quad$ Remanent flux density

$C_{\text {ext }} \quad$ External capacitance

$C_{p a r}$ Parasitic capacitance

$C_{t o t}$ Total capacitance

$C_{t t} \quad$ Turn to turn capacitance

$D_{c} \quad$ Wire core diameter

$D_{o} \quad$ Wire outer diameter 


\section{$D_{t} \quad$ Coil diameter}

$f_{\text {exc }} \quad$ Excitation frequency

$f_{\text {res }} \quad$ Resonating frequency

$g \quad$ Geometric ratio between the length and the cross-sectional area of the permanent magnet

$H_{c} \quad$ Coercive force

$H_{m} \quad$ Operating field strength of the permanent magnet

$H_{c i} \quad$ Intrinsic coercive force

$H_{\text {source }}$ Coercivity of the permanent magnet

$i \quad$ Current

$i_{\text {coil }} \quad$ Current passing through the magnetic coil

$J \quad$ Total current density

$j \quad$ Imaginary number

$l \quad$ Length

$l_{h} \quad$ Thickness of the pump housing

$l_{s} \quad$ Secondary air gap to accommodate the Hall-effect sensor

$l_{t} \quad$ Length of one wire turn on coil

$l_{c g} \quad$ Constant gap width between the impeller and the stuffing box

$L_{\text {coil }} \quad$ Inductance of the magnetic coil

$l_{\text {coil }} \quad$ Length of the magnetic coil

$l_{c} \quad$ Total length of the flux guide assembly

$l_{\text {gap }} \quad$ Width of the air gap 


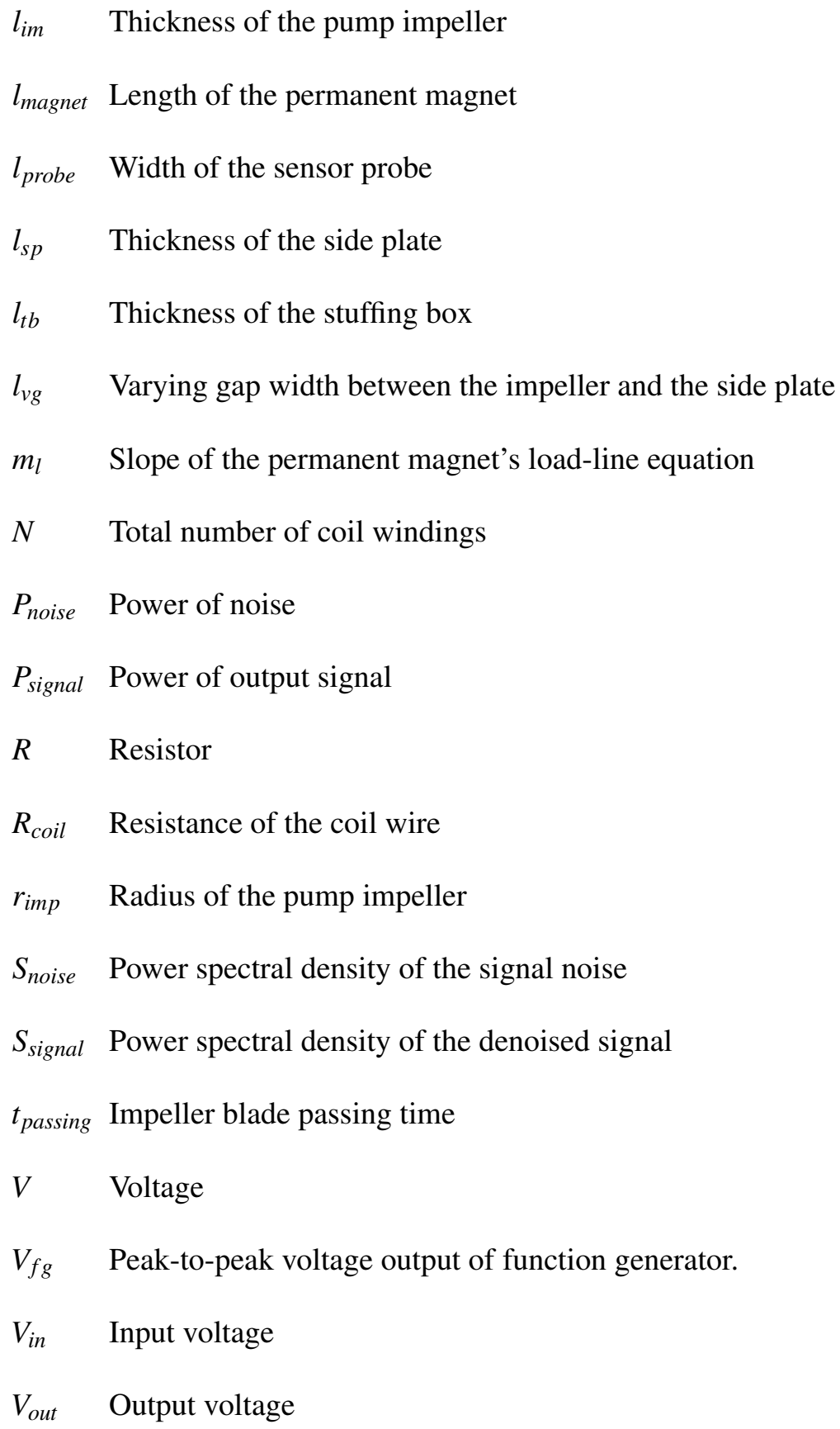




\title{
Acknowledgments
}

\begin{abstract}
Above all, I would like to express my sincere gratitude to my supervisor Dr. Boris Stoeber for his invaluable insight, guidance, encouragement and most importantly for believing in me through out the course of this project especially the times where I was left helpless and lost. The product, is the greatest accomplishment of my life and for this I'm forever thankful.
\end{abstract}

I am also deeply grateful to my co-supervisor Dr. Bhushan Gopaluni for his help and support in this project. His one of a kind problem solving skills has helped me become a better engineer at work and a better person in life. I would like to thank Meaghan Miller, our research engineer, for all the help she has provided in this project. I also appreciate the help of Glenn Jolly and Sean Buxton in the instrumentation shop for their technical assistance and for scaring away the electrons to go where they were supposed to.

I extend a most genuine thank to my dear lab mates. It was for their encouragement and support that I was able to reach this milestone. To Maziyar Jalaal in particular, for his unconditional help and support every time I asked. I shared with him the happiest and the toughest moments of this journey. I also owe a big thank you to my dear friends, the Seyeds, my roommate Sina, and my girlfriend Alexandra for being there when I needed them the most and for bearing with me on the days that my experiments would all fail.

And last but not least, a very special thank you to my family, for everything and anything that I have. 


\section{Dedication}

This thesis is dedicated to my only sister Taraneh who with her endless heart taught me how to live and how to love. I dedicate this to her for reasons beyond what I can ever articulate here. 


\section{Chapter 1}

\section{Introduction}

There was a door to which I found no key

There was a veil past which I could not see

Some little talk awhile of me and thee

There seemed... and then no more of thee and me.

- Omar Khayyam, Rubaiyat

\subsection{Motivation}

On average, centrifugal pumps consume between $25 \%$ and $60 \%$ of the total consumed electrical energy inside process plants depending on the industry[2]. Running pumps in an inefficient way is associated to high energy costs and therefore maintaining a high pump efficiency is becoming more and more vital for the economic viability of the industrial processes [3]. Improperly operated pumps are very common in the industry and are costing the companies excess amount of money to maintain[4]. Thus, any improvements to the operation of pumping systems will significantly reduce the total energy cost for plants.

Centrifugal pumps are the most common type of process pumps used in almost every industry[5]. One of the main factors that significantly affects the operating efficiency of a centrifugal pump is the presence of volumetric losses [6]. A major source of volumetric loss in an open-impeller centrifugal pump is the impeller 
clearance. The impeller inside a centrifugal pump is positioned between the side plate (or the housing depending on the type of the centrifugal pump) and the stuffing box as shown in Figure 1.1. The impeller clearance, hereinafter referred to as the varying gap, is identified as the gap width between the impeller and the side plate. When the impeller is moved away from the side plate, the varying gap increases leading to a significant drop in the pump's efficiency[7]. The main cause of this occurrence is wear being accumulated on the surface of the impeller over time. Wear typically occurs on the tip of the impeller blades[8] as shown in Figure 1.2. To estimate the magnitude of wear, measurements must be taken from the edges of the impeller blade as shown in Figure 1.3.

Depending on the material properties of the impeller and the consistency of the fluid being transported through the pump, impeller blades are considered fully worn within one to five years of operation. Subject to the severity of wear, impellers are either repaired or fully replaced during an overhaul of the equipment. The magnitude of the maximum allowable wear on the impeller blades ranges widely based on the size of the pump and its significance inside the plant. For a two blade openimpeller 14-inch diameter centrifugal pump made from cast iron, the magnitude of the maximum allowable wear is roughly $1.85 \mathrm{~mm}$.

To verify the working condition of an operating centrifugal pump inside a plant, wear accumulated on the tip of the impeller blade needs to be monitored while the pump is running. This will considerably improve the maintenance scheduling of the pump and prevent any unexpected erosion-related failure that may occur.

\subsection{Background}

Thus far there is no method for monitoring erosion inside industrial sized centrifugal pumps while the pump is in operation. Most of the studies that aim to measure and characterize wear have focused on wear on the surface of work tools and exposed rotating equipment. As such, a previous study has assessed the magnitude of wear on metallic surfaces through the deposition of a thin resistive film. As 


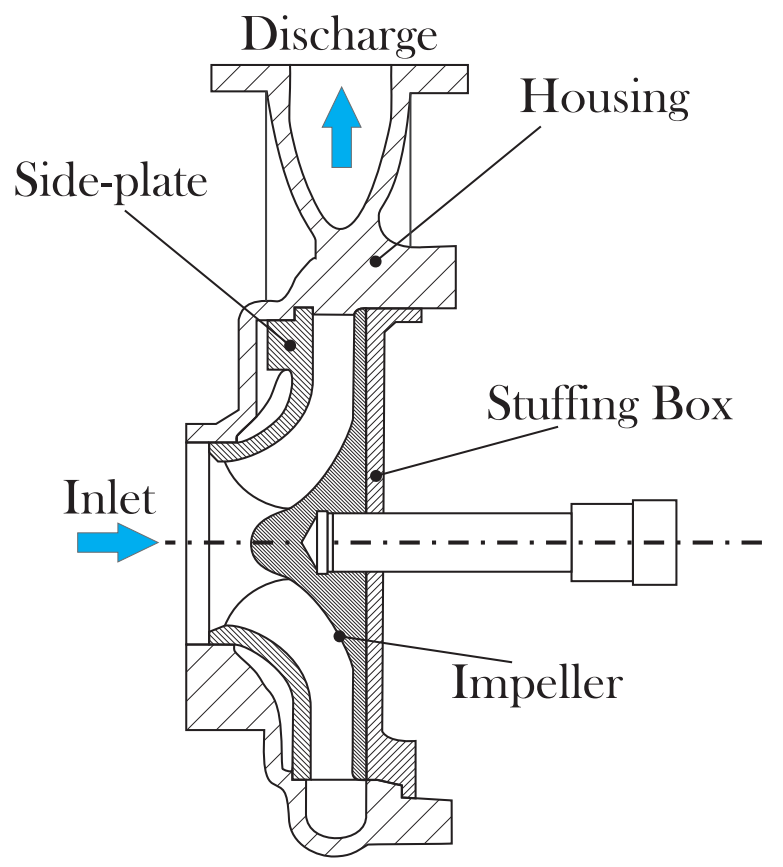

Figure 1.1: Cross-sectional diagram of a typical two blade open-impeller centrifugal pump.

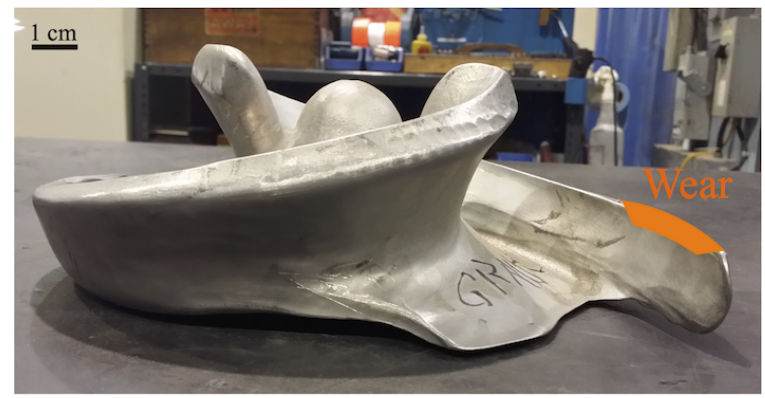

Figure 1.2: Two-blade pump impeller. The typical location of wear on the impeller is labeled on the figure. 


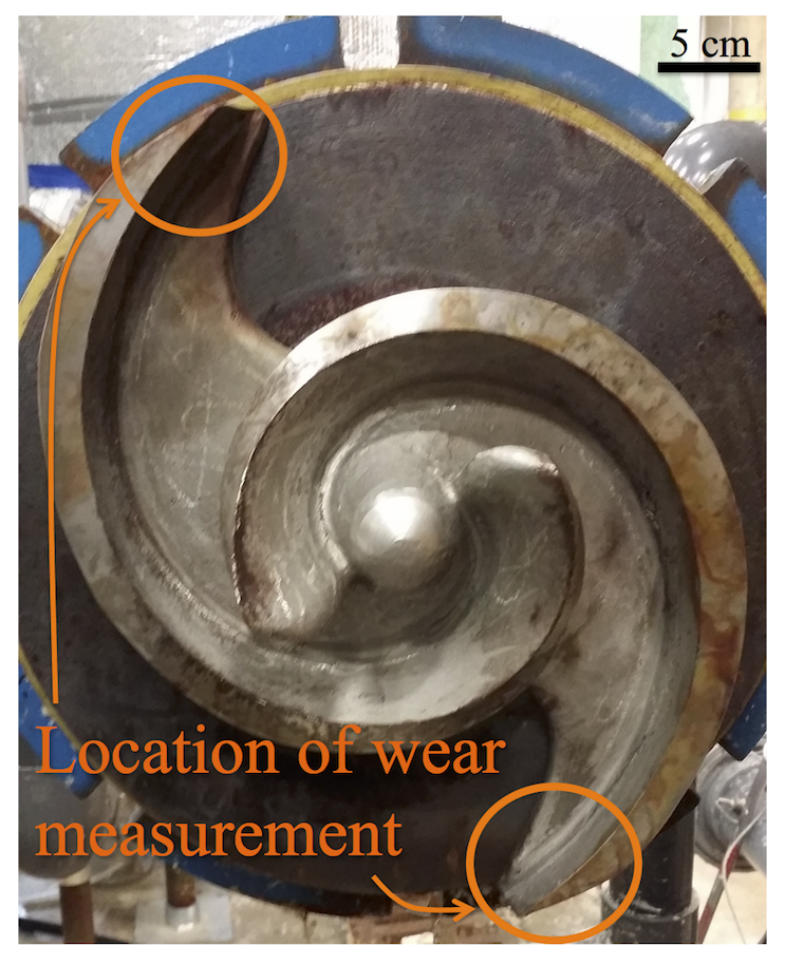

Figure 1.3: Top view of a two blade open impeller made from cast iron. The suitable location for wear measurement is labeled on the figure.

wear occurs, the resistance of the film increases, allowing for accurate wear measurement [9]. As one application of the film sensor, the study shows successful results for measuring tool wear on the surface of a cutting machine. This method, however, is not suitable for the aqueous environment of a pump. Also, the deposition of the thin resistive film on the surface of the material requires specialized tools which increases the cost of implementation. In another study related to tool wear, an automatic tool-wear sensor is proposed for a lathe machine application [10]. In this study, wear is measured through the use of a camera and the post processing of the captured images are used to characterize wear with an accuracy of $0.010 \mathrm{~mm}$. Since this method requires images to be taken from the surface of the tool, wear measurement on the blades of an operating pump impeller is not achievable through this technique. In another study, a more complex approach for measuring tool wear during a $\mathrm{CNC}$ milling process has been proposed [11]. In this 
study a neural network-based sensor fusion has been implemented to measure the cutting forces, vibration, spindle current, sound, and pressure during a milling process. This method has also been validated by an industrial implementation. This sensor network, however, is specifically designed for a CNC machine and its effectiveness for a centrifugal pump is not validated.

Another area of study related to this topic is a gap measuring tool. A method for measuring the gap width between two rotating plates can be used to estimate wear inside the equipment. One proposed approach for gap measurement inside rotating equipment is through the use of a capacitive or an inductive proximity sensor fixed inside the equipment [12], [13]. The patents for these two types of sensors claim a sensing gap range of $0.127 \mathrm{~mm}$ to $5.08 \mathrm{~mm}$. This method is being widely used in the industry for measuring the gap size between refiner plates inside a pulp refiner. Although this method can produce very accurate results, it requires the modification of the equipment to house the sensor which is an expensive process and is likely to alter the performance of the equipment. This is very critical in the case of a centrifugal pump. In a much smaller scale, a fiber-optic gap sensor is proposed to be used on a rotary undulation pump. Undulation pumps are for use inside artificial hearts. This study proposes the use of two parallel plastic core fibers for measuring the location of the rotor with respect to the stator plate for its dynamic positioning inside the pump [14].

Lastly, wear inside rotating equipment has also been correlated to the acoustic noise generated while the equipment is in operation. This field of study is referred to as the acoustic emission (AE) and is being widely studied for equipment that need continuous monitoring while in operation. A study has performed a time series analysis of acoustic emission signals generated from a cutting tool through the use of an autoregressive time-series to model the acoustics generated during the cutting process [15]. This method is highly application specific and a complete analysis of the acoustic noise is required for every equipment. Also, the viability of this method on other rotating equipment such as a centrifugal pump is yet to be validated. In regards to the application of acoustic emission in centrifugal pumps, a study has shown the use of AE to detect cavitation inside centrifugal pumps and 
to identify their best efficiency point [16].

\subsection{Objectives}

The main objective of this project is to develop a wear measurement sensor to be used on centrifugal pumps inside process plants. The sensor shall detect the degradation of the pump impeller enabling the use of a predictive maintenance scheduling system to prevent catastrophic failure of the equipment.

The manufacturing cost of the mentioned sensor needs to be considerably lower than the periodic overhaul expenses that could be prevented through its use. The sensor should also provide a simple installation procedure on an operating centrifugal pump without further pump modification. Ideally the sensor would be installed on every critical pump inside a process plant and the readings are transferred directly to the control room. The displayed data from the sensor will indicate a change in the thickness of the impeller blade due to erosion to notify the operator of the status of the pump. The required accuracy and the resolution of the measurements vary depending on the dimensions and the operating conditions of the pump and its components. For the 14-inch diameter two blade open-impeller centrifugal pump, as mentioned in Section 1.1, it can be safely assumed that resolving the full range of wear by $20 \%$ is sufficient to develop a predictive maintenance schedule and prevent costly breakdowns. Given that the pump has a maximum allowable wear of $1.85 \mathrm{~mm}$, the sensor needs to achieve an accuracy and a resolution better than $0.37 \mathrm{~mm}$. Since wear occurs very slowly during a period of several months, the response time of the sensor is not an important factor.

For the purpose of this project, the sensor is designed and tested on the 2-blade open impeller 40-Hp Westcan centrifugal pump currently installed at the UBC's Pulp and Paper pilot plant as shown in Figure 1.4. 


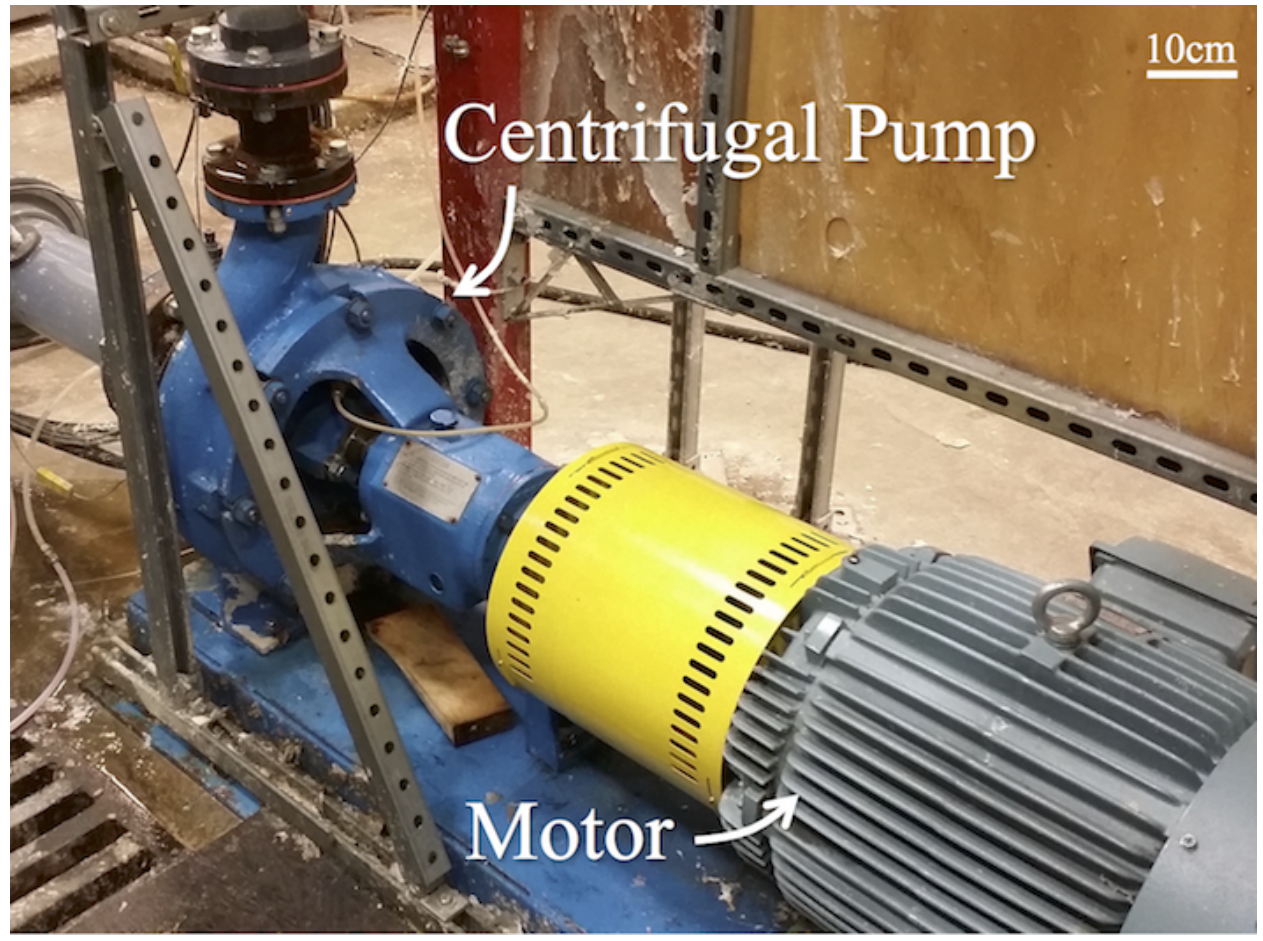

Figure 1.4: Westcan 40-Hp two blade open impeller centrifugal pump installed in the Pulp and Paper Center pilot plant at The University of British Columbia. The motor connected to the pump is also shown in this picture. 


\section{Chapter 2}

\section{Sensor Design}

To measure wear inside centrifugal pumps, a magnetic wear sensor is proposed. This chapter will start by summarizing the relevant background in magnetism that covers the principles of the magnetic circuit that is used to measure the varying gap width described in Section 1.1. The rest of the chapter will explain different concept variations of the sensor, the sensing method used, the analytical validation, the finite element analysis of the sensor assembly, and finally the method for the assessment of the sensor output.

\subsection{Sensor Principle}

The proposed sensor uses the principles of a simple magnetic circuit to measure wear inside centrifugal pumps. A magnetic circuit is a closed path that allows magnetic flux to travel through. The main component of the circuit's body is typically made from highly permeable material to guide the flux and minimize the flux leakage. Here, the pump is placed inside the path of the circuit with the varying gap inside the pump between the impeller and the side plate being part of the circuit. A simple analytical model of the magnetic circuit is created to evaluate the behaviour of the sensor with respect to a change in the size of the varying gap width.

The flux traveling inside a magnetic circuit is typically generated by the use 
of a permanent magnet or an electromagnet such as a magnetic coil. The magnitude of the flux carried inside the circuit can be derived from two of Maxwell's equations[17]. According to Ampère's Law

$$
\oint \vec{H} \cdot \mathrm{d} l=\int J \cdot \mathrm{d} A=i
$$

the closed line integral of the magnetic field strength $\vec{H}$ over the length of the magnetic circuit $l$ equals the total electrical current $i$ through the area defined by the closed line integral. The second fundamental equation

$$
\oint \vec{B} \cdot \mathrm{d} A=0
$$

describes the conservation of magnetic flux. This implies that the integral of the magnetic flux density over the surface area $A$ of any enclosed volume must be equal to zero. The magnetic field strength inside the circuit and the flux density are related through the permeability $\mu$

$$
\vec{B}=\mu \vec{H}
$$

The magnetic permeability $\mu$ represents the magnetization of the material used inside the circuit in response to an applied magnetic field. The magnitude of permeability

$$
\mu=\mu_{0} \mu_{r}
$$

can be written as the permeability of free space $\mu_{0}=4 \pi * 10^{-7} \mathrm{H} / \mathrm{m}$, multiplied by the relative permeability of the desired medium $\mu_{r}$.

In the case of a simple magnetic circuit, there is a single flux source driving the magnetic flux through the circuit. There is also a medium that provides a path for the magnetic flux, referred to as the flux guide throughout this document. Since the pumps considered in this project are generally used to transport water or water with pulp suspension, and given that the relative permeability of water and potential pulp consistency is very close to that of the air $\left(\mu_{r, \text { water }} \cong \mu_{r, \text { wood }} \cong \mu_{r, \text { air }} \cong 1\right)$, the gap inside the pump is replaced by a gap in the simplified magnetic circuit as shown in 


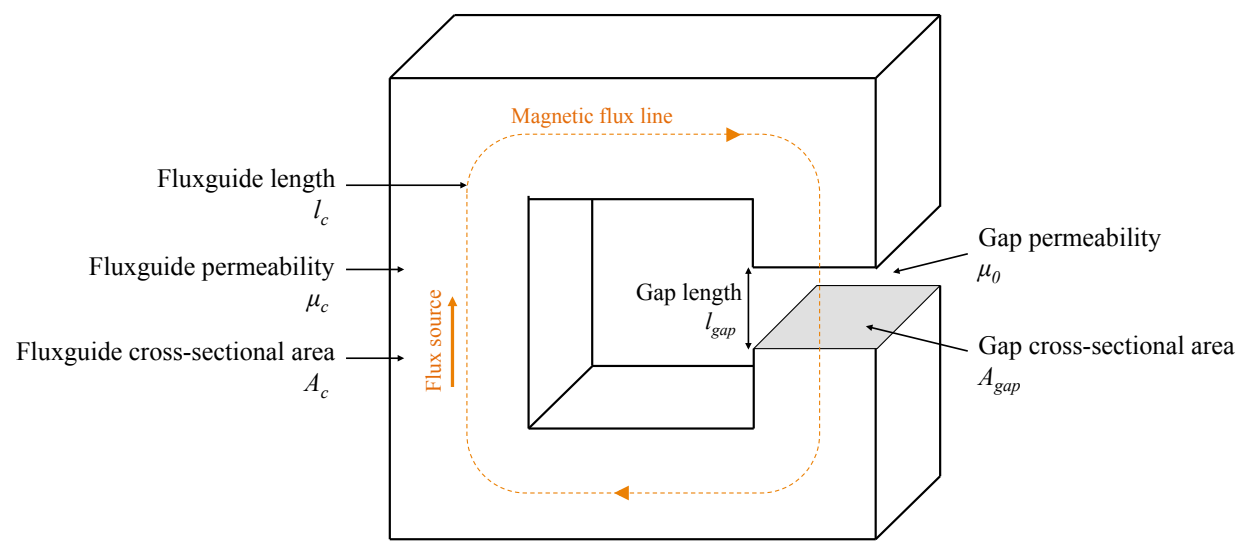

Figure 2.1: The diagram of a simple magnetic circuit with a single gap.

Figure 2.1. Therefor the line integral of the magnetic field strength, as represented in Equation 2.1, over the length of the circuit

$$
\sum H \cdot \Delta l= \begin{cases}0, & \text { for permanent magnet } \\ N \cdot i_{\text {coil }}, & \text { for magnetic coil }\end{cases}
$$

is equal to zero for the case or the permanent magnet as a flux source, and $N \cdot i_{\text {coil }}$ for the magnetic coil, where $i_{\text {coil }}$ represents the current passing through the windings of the coil with $N$ number of turns. The magnitude of the flux density inside the flux guide is also dependent on the source of the magnetic flux. By solving Equations 2.1 through 2.6 , the magnetic flux density

$$
B_{\text {source }}= \begin{cases}-\frac{\frac{l_{\text {magnet }}}{A_{\text {magnet }}}}{\frac{l_{\text {gap }}}{\mu_{0} A_{\text {gap }}}+\frac{l_{c}}{\mu_{0} \mu_{c} A_{c}}} H_{\text {source }}, & \text { magnet } \\ \frac{N}{A_{\text {coil }}} & \text { coil } \\ \frac{l_{\text {gap }}}{\mu_{0} A_{\text {gap }}}+\frac{l_{c}}{\mu_{0} \mu_{c} A_{c}} & \end{cases}
$$

can be written as a function of the flux guide parameters with a relative permeability of $\mu_{c}$, cross-sectional area of $A_{c}$ and a length of $l_{c}$, and the parameters of the gap with a relative permeability of $\mu_{r}=1$, cross-sectional area of $A_{\text {gap }}$ and a length 
of $l_{\text {gap }}$. For the case of the permanent magnet, the equation also depends on the geometry and material properties of the permanent magnet used. In this case the magnet's length $l_{\text {magnet }}$, cross-sectional area $A_{\text {magnet }}$, and the magnet's coercivity $H_{\text {source }}$. Similarly for the case of the magnetic coil, the equation depends on the coil's number of windings $N$, cross-sectional area $A_{\text {coil }}$ and current $i_{\text {coil }}$. It can be observed that in both cases the generated flux density $B_{\text {source }}$ is inversely proportional to the width of the air gap $l_{\text {gap }}$. This equation can also be written in terms of the circuit's reluctance value

$$
\Re=\frac{l}{\mu A} .
$$

Reluctance is an indicator of the capacity for storage of magnetic energy. It is both a function of geometry and material properties for each element inside the circuit. Equation 2.9 can be used to further simplify Equations 2.7 and 2.8 so that the magnetic flux density

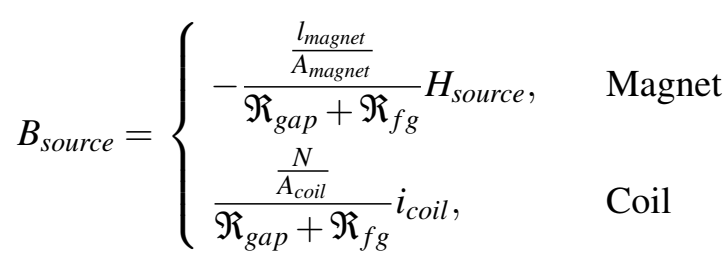

can be written in terms of the reluctance of the flux guide $\Re_{f g}$ and the reluctance of the gap $\Re_{\text {gap }}$. An increase in the width of the gap inside the circuit, increases the reluctance of the gap which results in an increase in the total reluctance of the circuit.

This situation is the same for the more realistic model of the pump. Figure 2.2 shows the schematic diagram of the centrifugal pump positioned axially in the path of the magnetic circuit with its components inside the path of the magnetic flux. As it can be seen from the drawing, the varying gap width inside the pump is also a component of the sensor and therefore a change in its reluctance will alter the total reluctance of the circuit. The geometry and the material properties of all the other components of the pump placed inside the path of the flux also affect the behaviour of the circuit. 


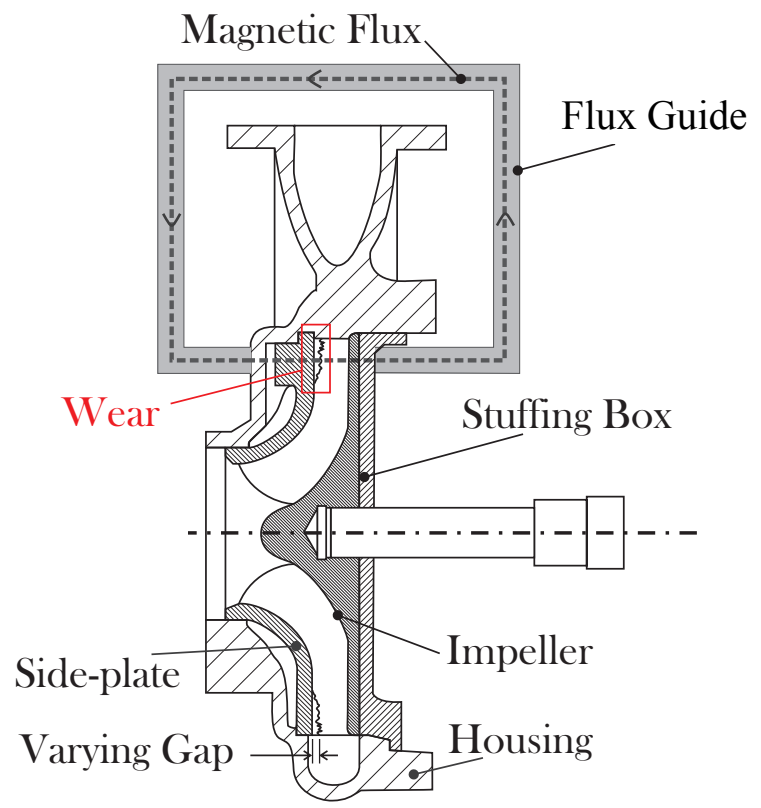

Figure 2.2: The schematic drawing of the magnetic circuit created with the pump and the flux guide assembly. The dotted line represents the path of the magnetic flux inside the circuit. The location of the wear on the tip of the impeller is indicated on the drawing.

As mentioned in Section 1.3, a two-blade 14-inch open impeller 40-HP centrifugal pump by Westcan is used for this study. All of the components of the pump are made from cast iron with a relative permeability of $\mu_{p}=500$. This is common for the majority of centrifugal pumps used inside process plants. To evaluate the performance of the sensor, the thickness of the pump housing $l_{h}$, the stuffing box $l_{t b}$, the side-plate $l_{s p}$, and also the constant gap width between the stuffing box and the impeller $l_{c g}$ are physically measured. The thickness of the impeller $l_{i m}$ and also the varying gap width $l_{v g}$ vary as wear occurs on the blades of the impeller. The change in the magnitude of the varying gap between no-wear and maximum-wear states is identified by the pump manufacturer. For the purpose of validating the wear sensor concept, a simplified version of the pump is considered as shown in Figure 2.3. All the physical dimensions of the pump are presented in Table 2.1. Since the permeability of the impeller is much greater than the permeability of the 
Table 2.1: Physical dimensions of the pump components.

\begin{tabular}{ccc}
\hline Parameter & Symbol & Value \\
\hline Varying gap (no wear - maximum wear) & $l_{v g}$ & $0.65 \mathrm{~mm}-2.50 \mathrm{~mm}$ \\
Housing thickness & $l_{h}$ & $10 \mathrm{~mm}$ \\
Stuffing box thickness & $l_{t b}$ & $15 \mathrm{~mm}$ \\
Constant gap width & $l_{c g}$ & $0.762 \mathrm{~mm}$ \\
Side plate thickness & $l_{s p}$ & $11 \mathrm{~mm}$ \\
Impeller thickness & $l_{i m}$ & $38 \mathrm{~mm}$ \\
\hline
\end{tabular}

Table 2.2: Effective cross-sectional areas of the pump components.

\begin{tabular}{ccc}
\hline Effective area & Symbol & Value $^{1}$ \\
\hline Variable gap & $A_{v g}$ & $400 \mathrm{~mm}^{2}$ \\
Housing & $A_{h}$ & $10000 \mathrm{~mm}^{2}$ \\
Stuffing box & $A_{t b}$ & $10000 \mathrm{~mm}^{2}$ \\
Constant gap & $A_{c g}$ & $10000 \mathrm{~mm}^{2}$ \\
Side plate & $A_{s p}$ & $10000 \mathrm{~mm}^{2}$ \\
Impeller & $A_{i m}$ & $400 \mathrm{~mm}^{2}$ \\
flux guide & $A_{c}$ & $2000 \mathrm{~mm}^{2}$ \\
\hline
\end{tabular}

${ }^{1}$ The presented values are estimates based on the 3D simulation model.

gap (1:500), the change in the total reluctance of the sensor due to a change in the thickness of the impeller can be neglected. The flux guide assembly is also assumed to have a high relative permeability of $\mu_{c}=10,000$ and a length of $l_{c}$. The reasoning behind this selection will be explained in Section 2.3. To analytically model the behaviour of the magnetic circuit, the effective cross-sectional areas of all the components of the circuit are also taken into consideration. For this reason, the cross-sectional areas have been estimated by fitting a simple parametric model with the more realistic 3D model of the circuit in Comsol Multiphysics. The resulting estimated areas are shown in Table 2.2. 


\section{Flux guide}

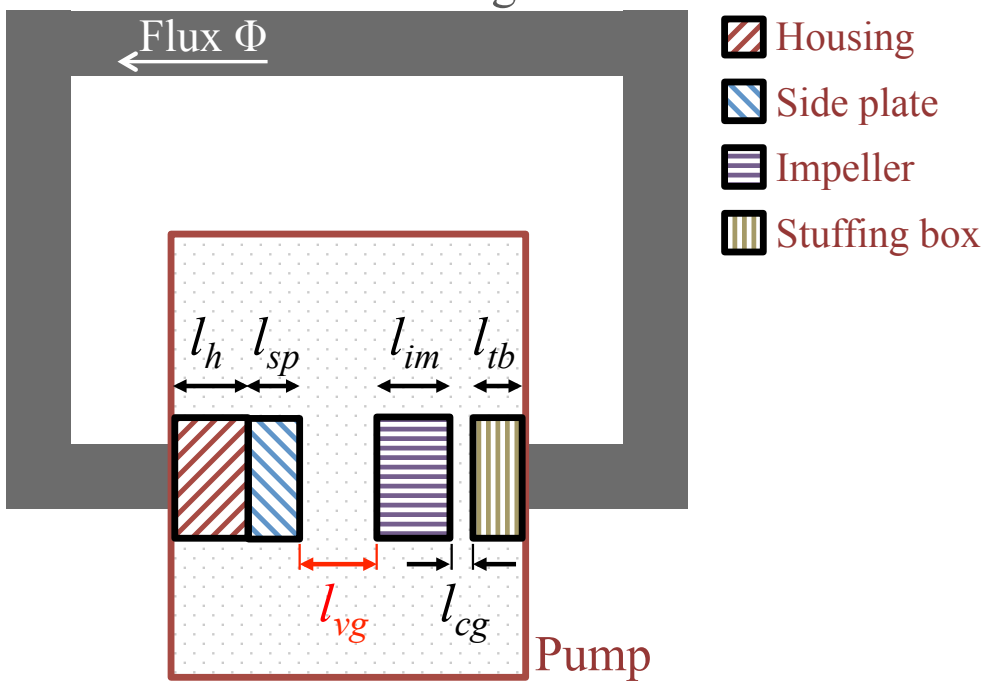

Figure 2.3: Simplified model of the pump and the sensor's flux guide with each component labeled on the diagram.

To measure the width of the varying gap inside the pump, the change in the total reluctance of the circuit is measured. The total reluctance of the circuit created with the pump

$$
\mathfrak{R}_{\text {total }}=\mathfrak{R}_{\text {var }}+\mathfrak{R}_{\text {const }}
$$

is composed of a varying reluctance referring to the varying gap width between the impeller and the side plate

$$
\Re_{v a r}=\frac{l_{v g}}{\mu_{0} A_{v g}}
$$

and a constant reluctance for the remaining components of the pump

$$
\begin{gathered}
\Re_{\text {const }}=\frac{l_{h}}{\mu_{0} \mu_{p} A_{h}}+\frac{l_{t b}}{\mu_{0} \mu_{p} A_{t b}}+\frac{l_{c g}}{\mu_{0} A_{c g}}+\frac{l_{s p}}{\mu_{0} \mu_{p} A_{s p}}+ \\
\frac{l_{i m}}{\mu_{0} \mu_{p} A_{i m}}+\frac{l_{c}}{\mu_{0} \mu_{c} A_{c}} .
\end{gathered}
$$


As wear occurs at the tip of the impeller blades, the magnitude of the varying reluctance is increased, leading to an increase in the total reluctance of the circuit. To monitor the circuit's total reluctance in real time, three methods have been proposed: (1) a permanent magnet model, (2) an electromagnet model, and (3) an inductance model. The characteristics of these methods are discussed and compared in the following section.

\subsection{Sensing Method Variation \& Comparison}

\subsubsection{Permanent Magnet Model}

In this approach, a rare-earth permanent magnet is used to drive magnetic flux through the circuit. Wear is estimated by monitoring the change in the magnitude of the flux density as the total reluctance of the circuit increases. The operation of this method is described in this section.

\section{Permanent Magnets}

Permanent magnets are commonly characterized by their magnetization curve as a function of an applied magnetic field. The resulting hysteresis curve is referred to as the BH curve and it represents the total energy that can be delivered by the permanent magnet [17]. To determine the characteristics of a magnet, both the normal curve and the intrinsic curve on the $\mathrm{BH}$ plot is considered as represented in Figure 2.4. The intrinsic BH curve is obtained by subtracting the flux density of the applied field $H$ in vacuum from the normal $\mathrm{BH}$ curve. As the applied field varies, so does the operating flux density of the magnet. The field required to reduce the flux density $B$ to 0 is called the coercive force or coercivity $H_{c}$. When this value is smaller than the intrinsic coercivity $H_{c i}$, the $\mathrm{BH}$ curve becomes entirely linear in the second quadrant of the $\mathrm{BH}$ curve with a slope of $\mu_{0}$. The value of $B$ when the applied field is reduced to zero is called the remanent flux density $B_{r}$ and is another fundamental property of the permanent magnet. For the design of the sensor, a rare-earth magnet is selected with a composition of neodymium-iron-boron (Ne- 


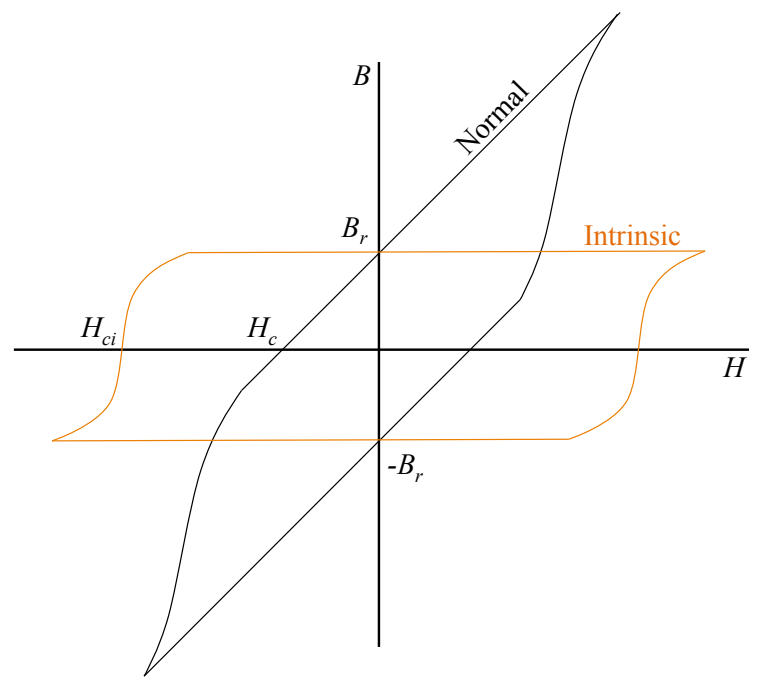

Figure 2.4: Magnetic hysteresis loop.

$\mathrm{FeB}$ ) also referred to as N42 with a remanent flux density of 1.3 Tesla. The use of this permanent magnet provides a linear relation between the flux density and the field strength of the magnet in the second quadrant of the $\mathrm{BH}$ curve also referred to as the demagnetization curve which helps further simplify the analytical model of the sensor.

\section{Analytical Modeling}

The operating point of a permanent magnet when placed inside a magnetic circuit can be determined by a combination of the circuit's load line equation and the magnet's demagnetization curve. The intersection of the two lines in the second quadrant of the BH curve will define the magnet's operating point as shown in Figure 2.5. Assuming the selected permanent magnet has a linear demagnetization curve in the second quadrant, as in the case of the N42 magnet, the demagnetization curve can be represented by the line equation

$$
B(H)=\mu_{0} H+B_{r}
$$

and the equation of the load line for the proposed sensor design 


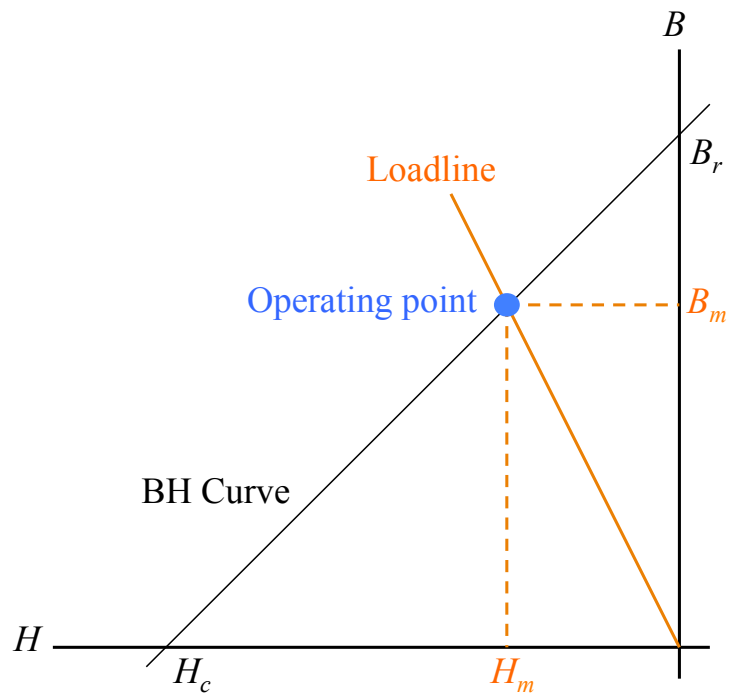

Figure 2.5: Demagnetization curve of a typical rare-earth magnet superimposed on the magnet's loadline.

$$
B(H)=-\frac{\frac{l_{\text {magnet }}}{A_{\text {magnet }}}}{\frac{l_{v g}}{\mu_{0} A_{v g}}+\Re_{\text {const }}} H .
$$

is derived from Equation 2.10. By solving Equation 2.15 and Equation 2.16, the magnet's operating flux density

$$
B_{m}\left(l_{v g}\right)=\frac{B_{r}}{1+\left(\frac{l_{v g}}{\mu_{0} A_{v g}}+\Re_{\text {const }}\right) \mu_{0} \frac{A_{\text {magnet }}}{l_{\text {magnet }}}}
$$

can be calculated as a function of the varying gap $l_{v g}$. It is observed that the operating point of the magnet varies as the varying gap width increases and this relationship can be used to measure wear. The most suitable method to measure the flux density inside the circuit is through the use of a Hall-effect sensor placed inside the path of the magnetic flux. Hall-effect sensors use the fundamental laws of magnetism to convert the magnitude of flux density to a voltage signal. For this project, an analog Hall-effect sensor was selected from Analog Devices (AD22151) with a sensitivity of $4 \mathrm{~V} / \mathrm{T}$ when excited using a $6 \mathrm{~mA}$ current. The full scale range of 
the sensor is $1.25 \mathrm{~T}$ with an adjustable offset and a non linearity error of $0.1 \% \mathrm{FS}$. Since the sensor needs to be positioned inside the path of the flux, a secondary air gap must be created in the flux guide assembly to accommodate the sensor. For this reason, Equation 2.17 is revised into

$$
B_{m}\left(l_{v g}\right)=\frac{B_{r}}{1+\left(\frac{l_{v g}}{\mu_{0} A_{v g}}+\frac{l_{s}}{\mu_{0} A_{s}}+\Re_{\text {const }}\right) \mu_{0} \frac{A_{\text {magnet }}}{l_{\text {magnet }}}}
$$

where $l_{s}=2 \mathrm{~mm}$ is the gap width required to fit the sensor and $A_{s}=4 \mathrm{~mm}^{2}$ is the cross-sectional area of the secondary gap that is determined from the working area of the sensor to ensure the sensor captures all the flux traveling through the circuit.

The response of the sensor can then be observed by solving Equation 2.18 for $B_{m}$ as a function of the varying the gap width $l_{v g}$. The geometric parameters of the permanent magnet used in this equation have been optimized to capture the highest sensitivity possible. The relevant equations for the geometry optimization of the magnet are presented in Appendix C. According to the geometric optimization, the ideal ratio between the magnet's length $l_{\text {magnet }}$ and the cross-sectional area $A_{\text {magnet }}$

$$
g=\frac{l_{\text {magnet }}}{A_{\text {magnet }}}=\mu \sqrt{\Re_{i} \Re_{f}}
$$

is calculated as a function of the total reluctance of the circuit where $\Re_{i}$ refers to the reluctance of the circuit before wear and $\mathfrak{R}_{f}$ refers to the reluctance when maximum wear is observed. For this case, the ratio $g$ was calculated to be $9 \mathrm{~m}^{-1}$. The magnet's physical parameters used in the analytical model are shown in Table 2.3. Figure 2.6 shows the the magnet's operating flux density as a function of the varying gap width. As the gap width increases from $0.65 \mathrm{~mm}$ (no wear) to $2.50 \mathrm{~mm}$ (maximum wear), the magnetic flux density drops from $1.178 \mathrm{~T}$ to $0.9324 \mathrm{~T}$.

\section{Finite Element Simulation Results}

To derive the analytical equation for the behaviour of the sensor, many simplifying assumptions are made. To validate these assumptions, a 3D model of the simplified 
Table 2.3: Magnet's physical parameters.

\begin{tabular}{ccc}
\hline Parameter & Symbol & Value \\
\hline Length & $l_{\text {magnet }}$ & $0.018 \mathrm{~m}$ \\
Cross-sectional Area & $A_{\text {magnet }}$ & $0.002 \mathrm{~m}^{2}$ \\
Remanent flux density & $B_{r}$ & $1.3 \mathrm{~T}$ \\
\hline
\end{tabular}

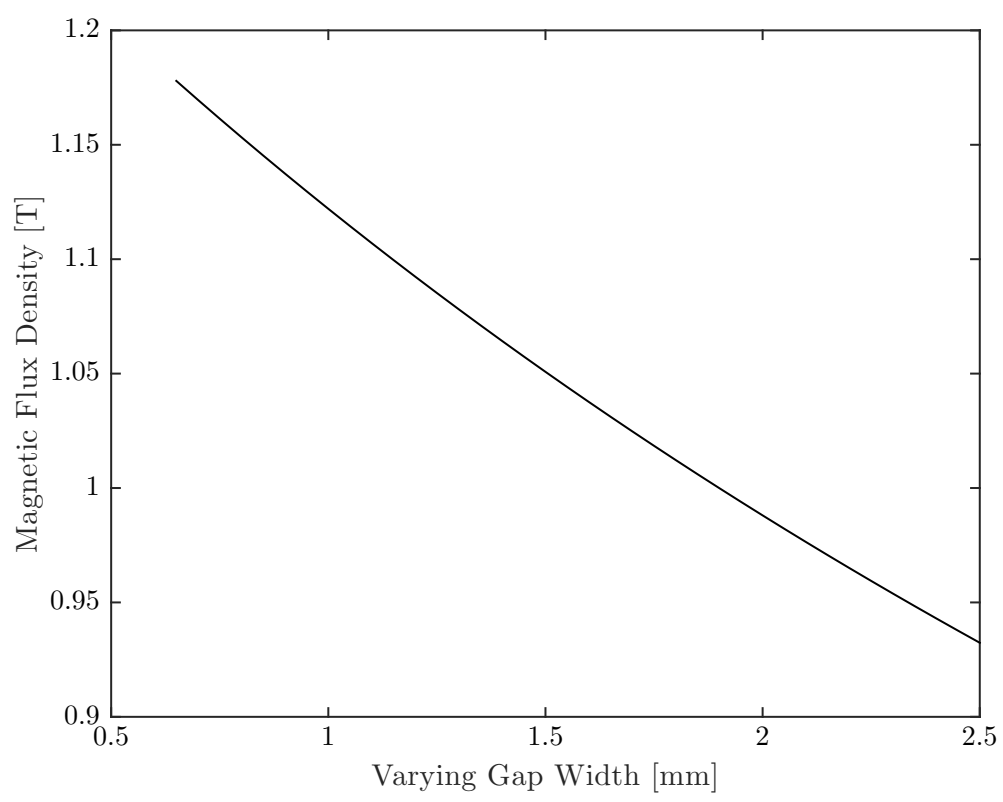

Figure 2.6: Plot of the operating magnetic flux density $B_{m}$ as a function of the varying gap width $l_{v g}$ as it increases from the no-wear state $(0.65 \mathrm{~mm})$ to the maximum-wear state $(2.50 \mathrm{~mm})$ for the analytical calculation using the permanent magnet model.

pump and the sensor is developed using Comsol Multiphysics shown in Figure 2.7 and the behaviour of the sensor is determined using this model. For the purpose of this investigation and to simplify the simulation results, the pump impeller is assumed to be stationary and positioned such that one impeller tip is in the magnetic flux path while the thickness of the impeller is reduced incrementally to account for wear inside the pump. The numerical analysis yields the magnetic flux density 
$B_{m}$ passing through the sensor as wear increases from no wear to maximum wear as shown in Figure 2.8.

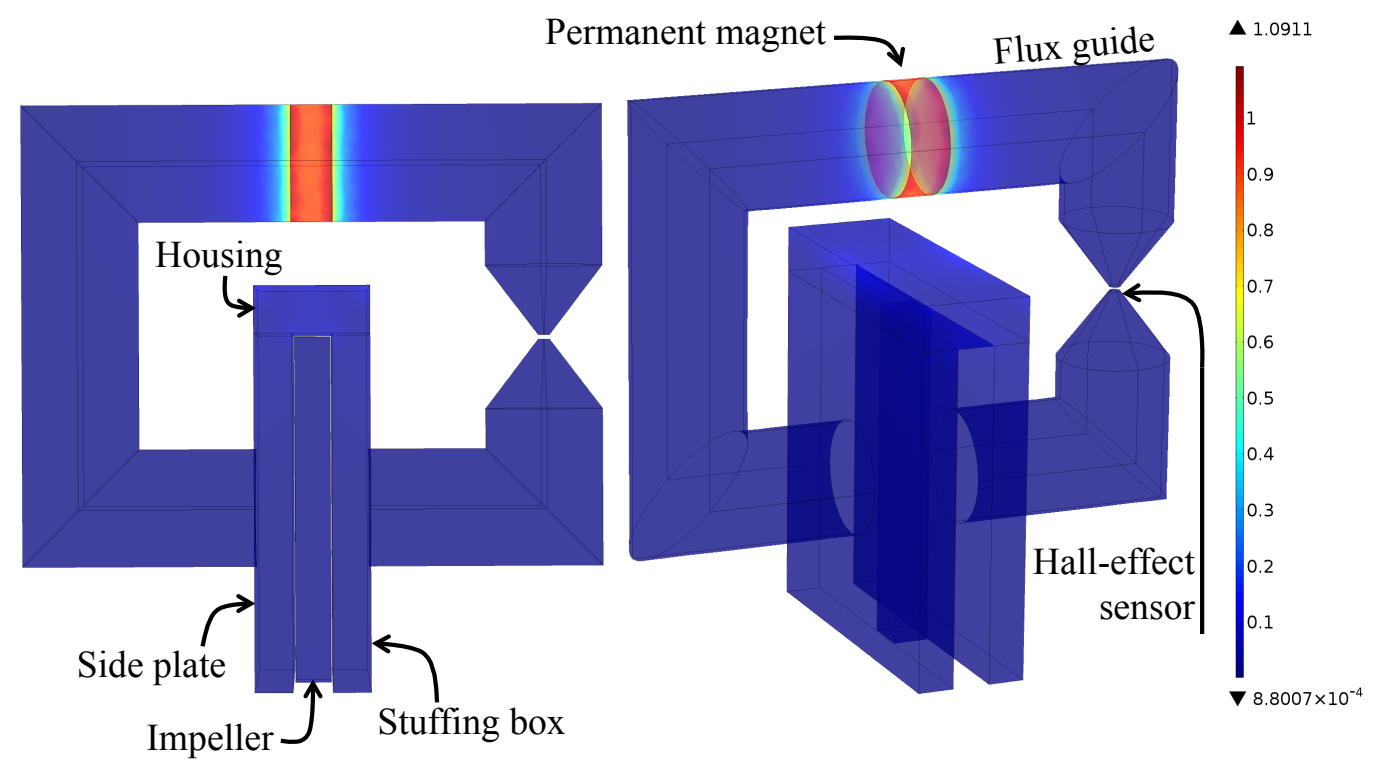

Figure 2.7: Magnitude of flux density [T] inside the sensor and the simplified pump for the analysis of the permanent magnet sensor model generated using Comsol Multiphysics.

The resulting transfer behaviour from the analytical calculation (Figure 2.6) and the finite element simulation in Comsol (Figure 2.8) are fairly similar in both magnitude and shape. The minor difference between the two models are primarily due to material saturation and flux leakage that exist in the simulation but are ignored in the analytical calculation. According to the simulation results, the flux density changes $0.51 \%$ as the varying gap inside the pump increases from $0.65 \mathrm{~mm}$ to $2.50 \mathrm{~mm}$. This indicates that the sensitivity achievable with the permanent magnet sensor model is $2.4 \mathrm{mT} / \mathrm{mm}$. By using the specified Hall-effect sensor with a sensitivity of $4 \mathrm{~V} / \mathrm{T}$, the sensitivity can be translated into $9.6 \mathrm{mV} / \mathrm{mm}$. 


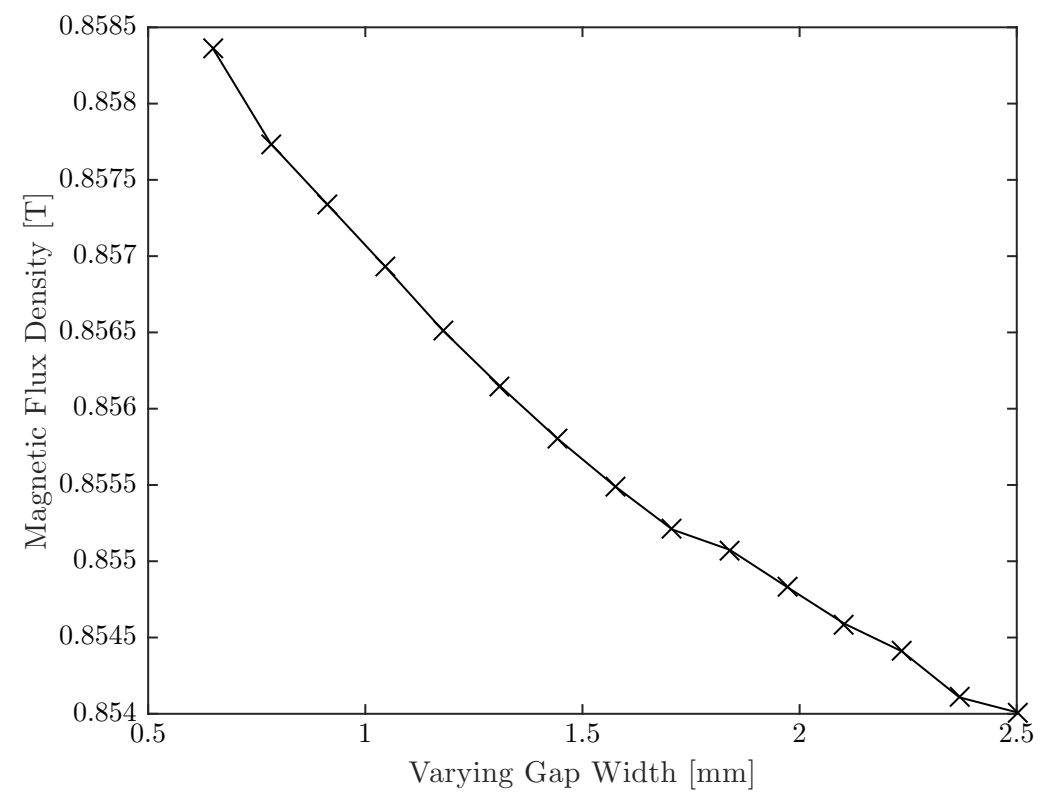

Figure 2.8: Plot of the magnetic flux density $B_{m}$ as a function of the varying gap width $l_{v g}$ for the case of the permanent magnet model derived from Comsol Simulation.

\subsubsection{Electromagnet Model}

Similar to the permanent magnet model, the electromagnet model also uses the change in the flux density to measure the width of the varying gap inside the pump caused by wear. However, instead of using a permanent magnet as a source of flux, an electromagnet is produced through the means of a magnetic coil placed on the flux guide assembly. The coil is connected to an AC voltage source to generate an alternating magnetic flux inside the flux guide. This allows for the fine tuning of the intensity of the magnetic flux to prevent saturation inside the flux guide material as observed in the case of the permanent magnet model. Also by applying an AC magnetic flux at a specific frequency, a large portion of the AC and DC noise can be filtered from the output of the Hall-effect sensor. On the down side, this model requires a more complex circuitry for data collection and analysis which makes the design less desirable. The rest of this section will go into the details of the ana- 
lytical modeling of the sensor and the numerical validation of the analytical results.

\section{Analytical Modeling}

As described in the beginning of this chapter, wear can be estimated by correlating the change in the varying gap width to the change in the reluctance of the magnetic circuit created with the pump. If a magnetic coil is used as the source of flux inside the circuit, then Equation 2.11 can be used to derive the magnitude of the flux density

$$
B_{\text {source }}\left(l_{\text {vg }}\right)=\frac{\frac{N}{A_{\text {source }}}}{\frac{l_{v g}}{\mu_{0} A_{v g}}+\Re_{\text {const }}} i_{\text {coil }}
$$

as a function of the varying gap width $l_{v g}$, and the coil parameters such as the cross sectional area $A_{\text {coil }}$, number of turns $N$ and the current passing through the coil windings $i_{\text {coil }}$. To measure the change in the total reluctance of the circuit in real time, the same Hall-effect sensor can be used as in the case of the permanent magnet model. Therefore, there needs to be an addition of a secondary air gap in the equation of magnetic flux to account for the sensor. This will change the relation for the magnetic flux density

$$
B_{\text {source }}\left(l_{\text {vg }}\right)=\frac{\frac{N}{A_{\text {coil }}}}{\frac{l_{\text {vg }}}{\mu_{0} A_{v g}}+\Re_{\text {const }}+\frac{l_{s}}{\mu_{0} A_{s}}} i_{\text {coil }}
$$

passing through the sensor to include the reluctance caused by the secondary air gap with the same dimensions as in the case of the permanent magnet. For the analytical calculations, the dimensions of the coil are selected so that the sensitivity of the sensor is maximized. The values used in the analytical model are represented in Table 2.4

For the analytical modeling of the sensor, MATLAB software is used to calculate the change in the magnitude of the flux density as wear causes an increase in the gap width inside the centrifugal pump. The result yields the magnitude of the flux density passing through the Hall-effect sensor as a function of the varying 
Table 2.4: Magnetic coil's physical parameters.

\begin{tabular}{ccc}
\hline Parameter & Symbol & Value \\
\hline Number of coil windings & $N$ & 1000 \\
Cross-sectional area & $A_{\text {coil }}$ & $0.002 \mathrm{~m}^{2}$ \\
Excitation current & $i_{\text {coil }}$ & $10 \mathrm{~mA}$ \\
Excitation frequency & $f_{\text {exc }}$ & $100 \mathrm{~Hz}$ \\
\hline
\end{tabular}

gap width as shown in Figure 2.9. It can be seen that the response of the sensor is very similar to the permanent magnet model as expected based on the analytical equations. In this case, the magnitude of flux density varies from $15 \mathrm{mT}$ to $12 \mathrm{mT}$ as the gap increases from no-wear state to the maximum-wear state.

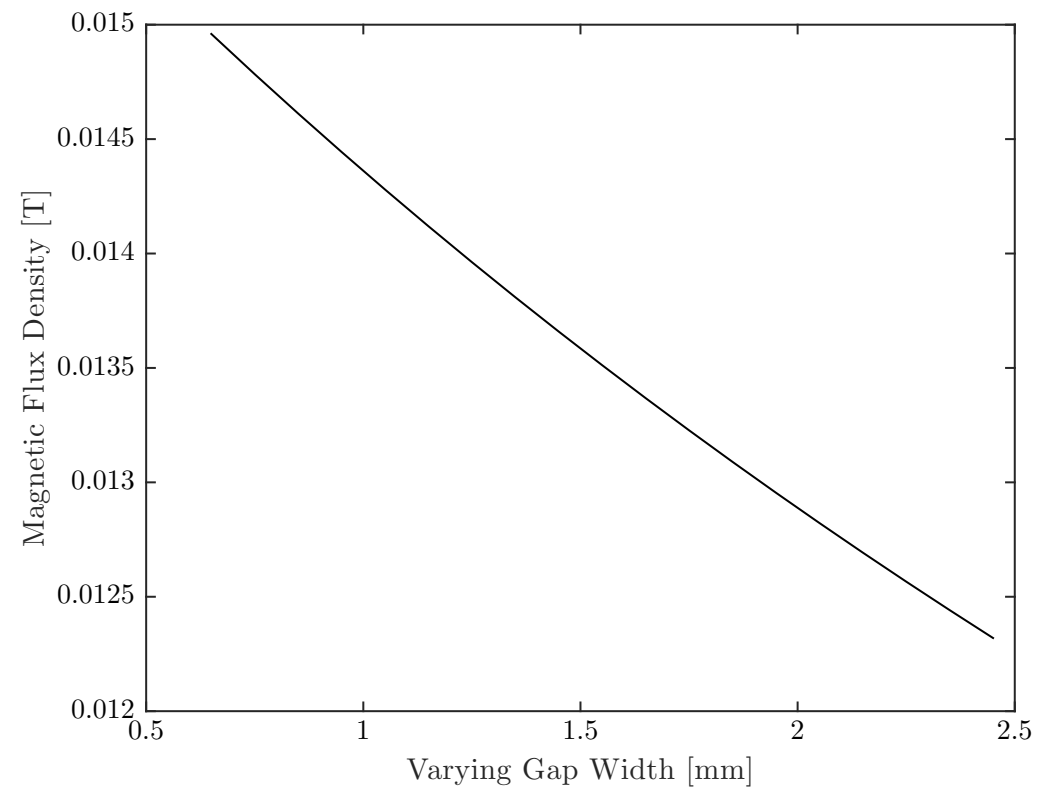

Figure 2.9: Plot of the operating magnetic flux density $B_{m}$ as a function of the varying gap width $l_{v g}$ as it increases from the no-wear state $(0.65 \mathrm{~mm})$ to the maximum-wear state $(2.50 \mathrm{~mm})$ for the analytical calculation using the electromagnet model. 


\section{Finite Element Simulation Results}

To validate the analytical results, a 3D model of the sensor is developed using the AC/DC module of Comsol Multiphysics software, represented in Appendix A, and the magnetic coil on the sensor is excited at $100 \mathrm{~Hz}$ with a current source of $10 \mathrm{~mA}$. Similar to the permanent magnet model, the impeller blades are assumed to be stationary to further simplify the simulation results. The numerical simulation yields the change in the flux density as a function of the varying gap width shown in Figure 2.10 . The results yield a sensitivity of $2.8 \mathrm{mT} / \mathrm{mm}$ that can be converted into $11 \mathrm{mV} / \mathrm{mm}$ using the same Hall-effect sensor as mentioned previously.

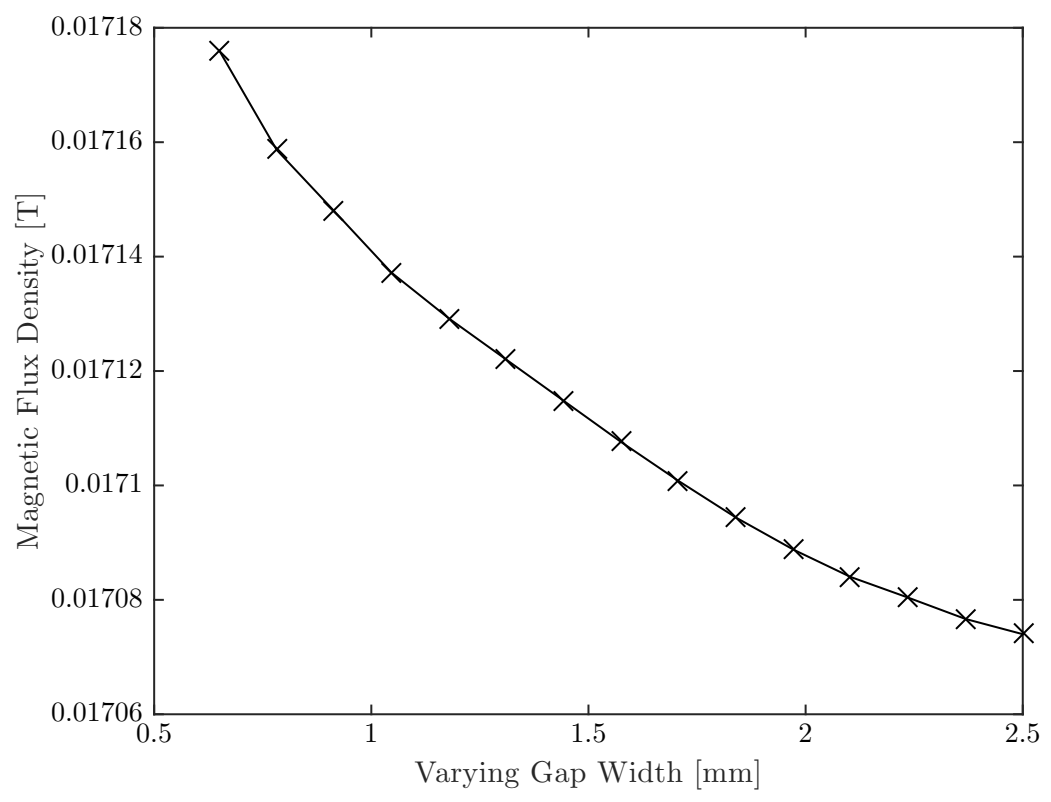

Figure 2.10: Plot of the magnetic flux density $B_{m}$ as a function of the varying gap width $l_{v g}$ for the case of the electromagnet model derived from Comsol Simulation. 


\subsubsection{Coil Inductance Model}

This method also requires the use of a magnetic coil to drive magnetic flux through the flux guide. The reluctance, however, is calculated by measuring the inductance of the coil as opposed to the magnitude of the flux density. This eliminates the need for a Hall-effect sensor to be placed inside the path of the magnetic flux and therefore eliminating the need for a secondary air-gap inside the flux guide. As a result, the total reluctance of the circuit is significantly reduced.

The inductance of a magnetic coil is a function of its core's reluctance value. Therefore, when a coil is placed on the flux guide assembly, the inductance of the coil

$$
L_{\text {coil }}=\frac{N^{2}}{\Re_{\text {total }}}
$$

can be written as a function of the number of coil windings $N$, divided by the total reluctance of the magnetic circuit [18]. This implies that as the total reluctance of the circuit increases due to wear, the inductance of the magnetic coil drops and this relation could be used to monitor wear while the pump is in operation. The rest of this section will go into the details of the analytical model of the sensor and the simulation results to validate the analytical calculations.

\section{Analytical Modeling}

The relationship between the coil's Inductance value and the circuit's total reluctance is used to analytically model the output of the sensor as wear occurs on the tip of the impeller blades inside the pump. By combining Equation 2.12, Equation 2.13, Equation 2.14, and Equation 2.22, the inductance of the coil

$$
L_{\text {coil }}\left(l_{v g}\right)=\frac{N^{2}}{\frac{l_{v g}}{\mu_{0} A_{v g}}+\Re_{\text {const }}}
$$

can be written as a function of the gap width $l_{v g}$. The analytical calculation yields the change in the inductance of the magnetic coil as the gap width increases from 
$0.65 \mathrm{~mm}$ to $2.50 \mathrm{~mm}$. The result of this analysis is shown in Figure 2.11. It can be observed that the inductance changes from $12.3 \mathrm{mH}$ to $9.55 \mathrm{mH}$ as a result of the change in the varying gap size.

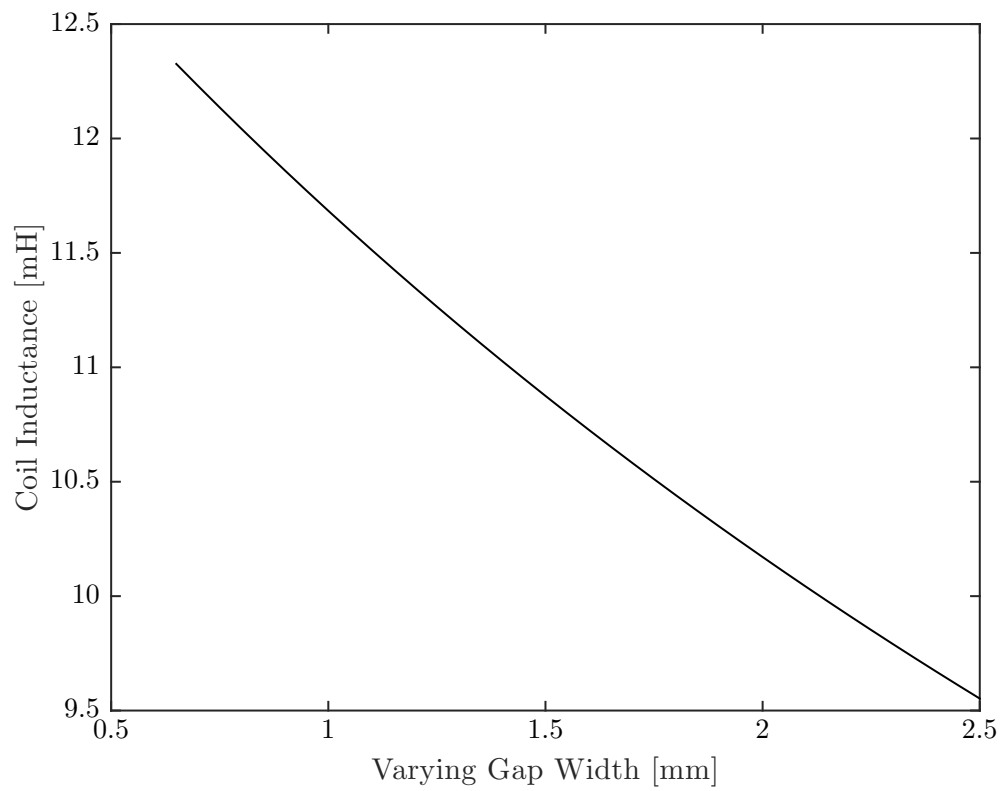

Figure 2.11: Coil inductance as a function of the varying gap width calculated using the analytical model of the coil inductance.

\section{Finite Element Simulation Results}

To further validate the analytical results of the inductance model, the pump and the sensor are modeled in a more realistic 3D geometry using Comsol Multiphysics as shown in Appendix A. Once again the AC/DC module of the Comsol Multiphysics is used to monitor the change in the inductance of the coil as the varying gap width inside the pump increases. Similar to the previous two models, the impeller blades of the pump are assumed to be stationary to further simplify the problem. The result of the simulation yields the inductance of the magnetic coil as a function of the varying gap width as shown in Figure 2.12. As the plot indicates, the inductance 
varies from $9.72 \mathrm{mH}$ to $8.96 \mathrm{mH}$ as the gap increases from $0.65 \mathrm{~mm}$ to $2.50 \mathrm{~mm}$.

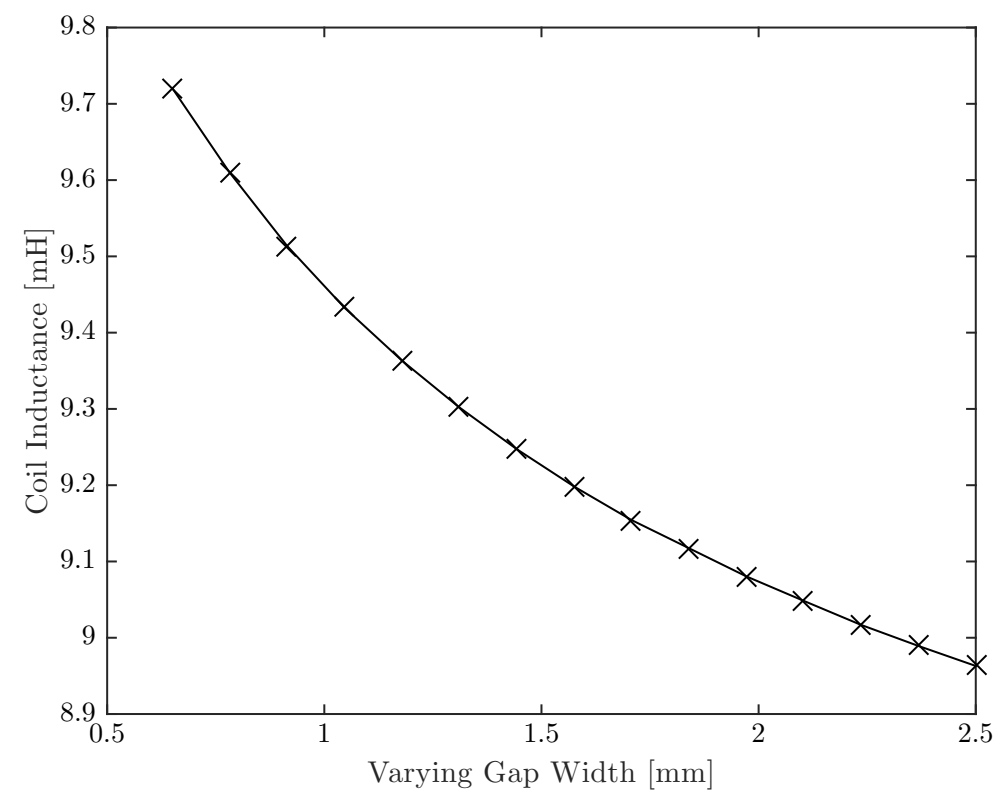

Figure 2.12: The inductance of the magnetic coil as a function of the varying gap width for the case of the inductance model derived using Comsol simulation.

\subsubsection{Method Selection}

In this chapter three different methods have been proposed for measuring the varying gap inside the pump, namely, the permanent magnet model, the electromagnet model, and the inductance model. To choose the most suitable approach, these methods are evaluated and compared based on the three most important design attributes as listed below in the order of importance:
a. Sensitivity
b. Signal to noise ratio
c. Sensor complexity 


\section{a. Sensitivity}

The sensitivity of the sensor is the most important parameter that needs to be taken into consideration. To compare the sensitivity of the three different models, the percentage change in the magnitude of the measuring parameter is calculated using the Comsol simulation results presented earlier. The plot of the percentage change versus the varying gap width is shown in Figure 2.13. As it can be seen from the generated plots, the responsiveness of the inductance model to a change in the varying gap width is much larger than the other two methods. This is primarily due to the absence of the Hall-effect sensor in the assembly and thus eliminating the need for a secondary air gap. It can also be observed that the electromagnet model operates slightly better than the permanent magnet model. This is due to material saturation at the sharp corners of the flux guide assembly in the case of the permanent magnet for the high magnetic flux density exerted by the permanent magnet.

\section{b. Signal to noise ratio}

The noise level inside a plant environment with the presence of motors and pumps operating in close vicinity is expected to be relatively high. The difference between the designs is in the effect of the existent noise on the output of the sensor. The main advantage of the electromagnet model and the inductance model over the permanent magnet model with regards to noise is in the use of an alternating magnetic field. Since an AC voltage signal is being used to operate the magnetic coil at a fixed frequency, the output signal can also be collected at the same excitation frequency to reduce the effect of any DC or AC noise apparent in the environment. Another potential source of noise inside a plant is the magnetic field from nearby permanent magnets that could exist in various coupling systems and motors. Since the permanent magnet model and the electromagnet model use a Hall-effect sensor to measure the change in the magnitude of the flux density, any neighboring magnetic field will add additional noise to the output of the sensor. 


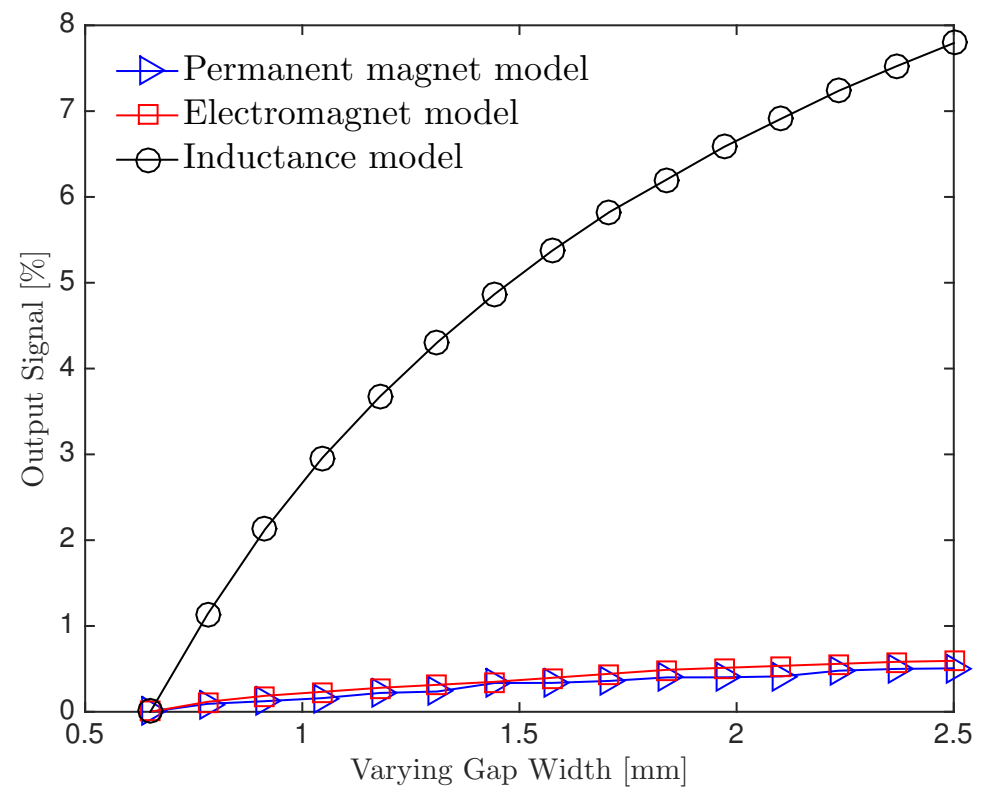

Figure 2.13: The percentage change in the measuring parameter as a function of the varying gap width for the permanent magnet model, electromagnet model, and the inductance model derived from Comsol simulations.

\section{Sensor complexity}

The complexity of the design of the sensor is also an important factor when it comes to selecting the most optimum design. The complexity could be in the physical fabrication of the flux guide assembly, or in the electrical circuitry required to operate the sensor. For the permanent magnet model and the magnetic coil model, the use of a Hall-effect sensor adds to the complexity of both the design of the flux guide assembly and to the circuitry required to operate the Hall-effect sensor.

\section{Conclusion}

In summary, the coil inductance model is the most suitable method for measuring the change in the varying gap width inside a centrifugal pump. Table 2.5 shows the 
comparison of the three different models based on the mentioned design attributes. Through the use of a magnetic coil, the flux generated inside the path of the circuit can be fine tuned to prevent any material saturation and the same coil can be used to monitor the change in the reluctance of the circuit. By using a single coil in the sensor, the design of the assembly and the circuitry is considerably simplified.

Table 2.5: The comparison of the three sensing methods.

\begin{tabular}{cccc}
\hline Design Attributes & $\begin{array}{c}\text { Permanent magnet } \\
\text { model }\end{array}$ & $\begin{array}{c}\text { Electromagnet } \\
\text { model }\end{array}$ & $\begin{array}{c}\text { Inductance } \\
\text { model }\end{array}$ \\
\hline Sensitivity & - & - & + \\
Signal to noise ratio & - & + & + \\
Sensor complexity & + & - & + \\
\hline
\end{tabular}

\subsection{Flux Guide Design}

The flux guide assembly is one of the key components of the sensor and its design will significantly affect the sensor's performance. The two most important parameters for the design of the flux guide assembly are the material properties and the physical geometry. The rest of this section will further explain the design process of the flux guide for an optimized performance.

\subsubsection{Material Selection}

The material used for the fabrication of the flux guide assembly plays an important role in the performance of the sensor. The most critical parameters that need to be considered when selecting the right material are the magnetic permeability, cost, and in some cases the saturation flux density.

For identifying the magnetic permeability required for the flux guide assembly, the effect of permeability is analyzed on the output of the sensor. The analytical model of the sensor is used to calculate the percentage change in the output signal using various material permeability values. As shown in Figure 2.14, when the 
magnetic permeability of the flux guide material increases, the sensitivity of the sensor is significantly improved. The improved sensitivity due to an increase in the material permeability saturates at just below $\mu_{r}=10,000$. This indicates that there is no significant improvement to the sensor once the permeability of the material exceeds this limit.

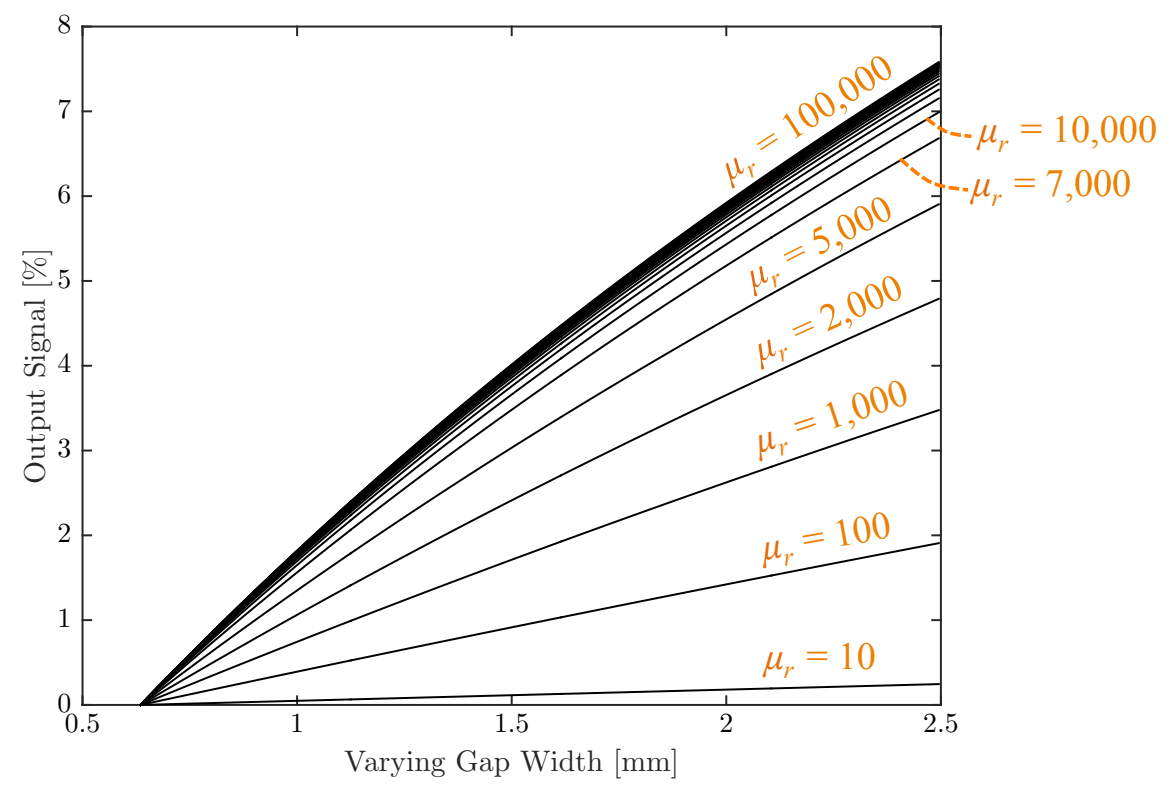

Figure 2.14: The percentage change in the inductance of the coil (output signal) using various magnetic permeability values for the flux guide assembly derived from the analytical model.

To select the most suitable material for the fabrication of the flux guide assembly, several alternatives are considered and compared based on their magnetic permeability, saturation flux density and cost as shown in Table 2.6. It is important to mention that the materials considered are also shortlisted based on their availability and delivery time. Based on the characteristics of the materials selected and the design parameters required to operate the sensor, the high-permeability VimVar material and the M100 material are the most suitable options for the fabrication of the flux guide assembly. Since the M100 material has an initial permeability also 
equal to its highest permeability of $\mu_{r}=10,000$, it does not require a biased field to be applied to the material to reach the highest possible permeability as in the case for the VimVar material. This further simplifies the fabrication process of the sensor and the instrumentation required. The M100 is a brand name for the Manganese Zinc Iron Ferrite material manufactured by National Magnetics Group Incorporation in the United States.

Table 2.6: High-permeability magnetic materials for the fabrication of the flux guide assembly.

\begin{tabular}{cccc}
\hline Material & Relative permeability $^{1}$ & Saturation flux density & Cost per mass $^{2}$ \\
\hline M100 & 10,000 & $0.42 \mathrm{~T}$ & $\$ 67.24 / \mathrm{kg}$ \\
VimVar & 10,000 & $2.15 \mathrm{~T}$ & $\$ 65.17 / \mathrm{kg}$ \\
Hiperco 50 & 15,000 & $2.42 \mathrm{~T}$ & $\$ 251.33 / \mathrm{kg}$ \\
Hy-Mu 80 & 230,000 & $0.87 \mathrm{~T}$ & $\$ 81.35 / \mathrm{kg}$ \\
\hline
\end{tabular}

${ }^{1}$ Relative permeability refers to the maximum permeability achievable from the material. For the case of the M100 material, this value is referring to the initial permeability which is also the maximum achievable.

2 The total weight of the flux guide assembly is roughly $30 \mathrm{~kg}$ and the densities for the selected materials are very similar.

Since the M100 material is very expensive compared to the cost of other components of the sensor, a study is done to analyze the behaviour of the sensor when the usage of this material is reduced by creating a composition of high-permeability M100 material and a cheaper alternative such as Carbon Steel with a relative permeability of 100 [19]. The purpose of this study is to identify a potential alternative assembly with a similar sensitivity except by using the M100 material on only specific parts of the assembly. However, as Figure 2.15 implies, the sensitivity of the sensor is only maximized when the entire assembly is made from the highpermeability M100 material. 

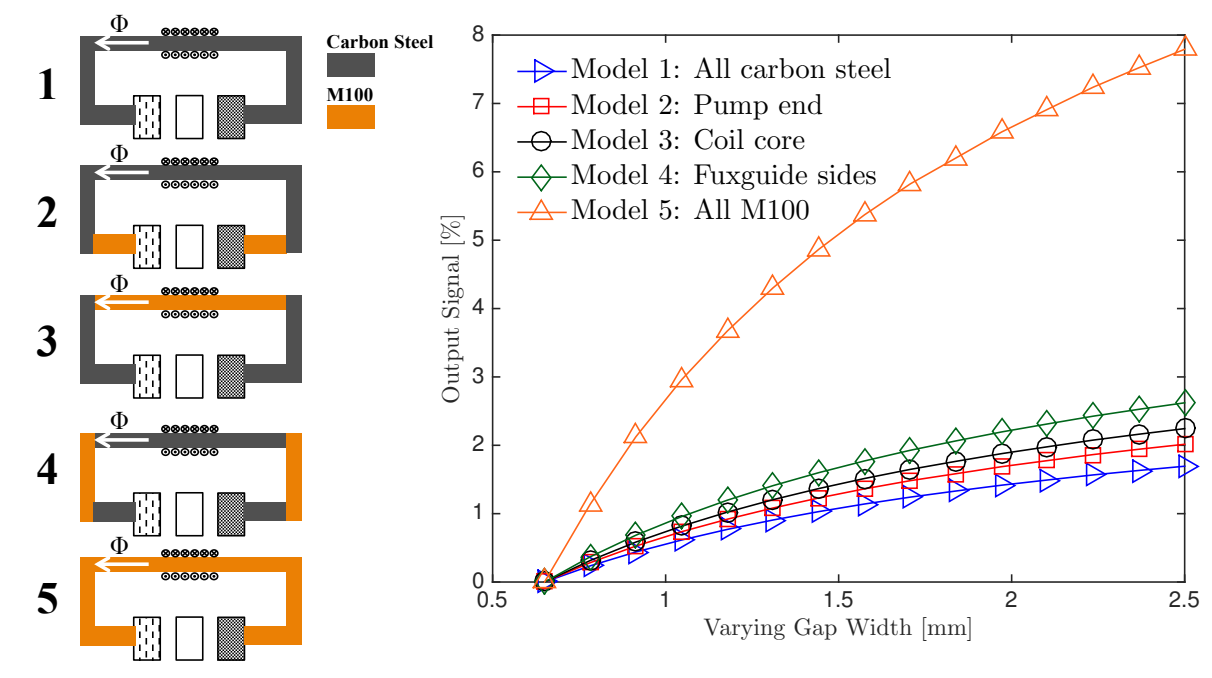

Figure 2.15: Sensitivity comparison of alternative material compositions for the flux guide assembly using carbon steel and high-permeability M100 material derived from Comsol simulations.

\subsubsection{Sensor Geometry}

The design of the flux guide geometry is vital for the operation of the magnetic wear sensor. Since the flux guide assembly constitutes the body of the sensor, its design should be easy to fabricate, easy to install, durable, rigid, and most importantly it should maximize the sensitivity of the sensor. The design should also take into account the cost of the material and the machining requirement in order to minimize the total cost of the sensor.

\section{Parameter Optimization}

The performance of the sensor is highly dependent on the geometry of the flux guide assembly. The geometry must provide the most ideal path for the flux field to pass through while creating a clamping mechanism for the sensor to be installed on any centrifugal pump inside a plant. The two most important design parameters for the geometry are the length and the cross-sectional area of the flux guide. Since both of these parameters exist in the equation of the circuit's total reluctance and 
given that by lowering the reluctance the sensitivity of the sensor can be improved, it can be expected that a shorter length and a larger cross-sectional area may refer to a higher sensitivity. To find the most optimum design, the analytical model of the sensor is used to calculate the change in the output signal as the design parameters are varied. The sensitivity is compared against varying the length of the assembly as shown in Figure 2.16, and the cross-sectional area as shown in Figure 2.17. As expected, the most ideal design is the one with the shortest length and the largest cross-sectional areal. However, since the assembly needs to be wrapped around a centrifugal pump, there is a minimum length requirement that limits the sensitivity improvement. Also, given that the material is valued at a cost per weight, the larger cross-sectional area refers to a higher cost and thus there is a trade-off between the sensitivity and the cost.

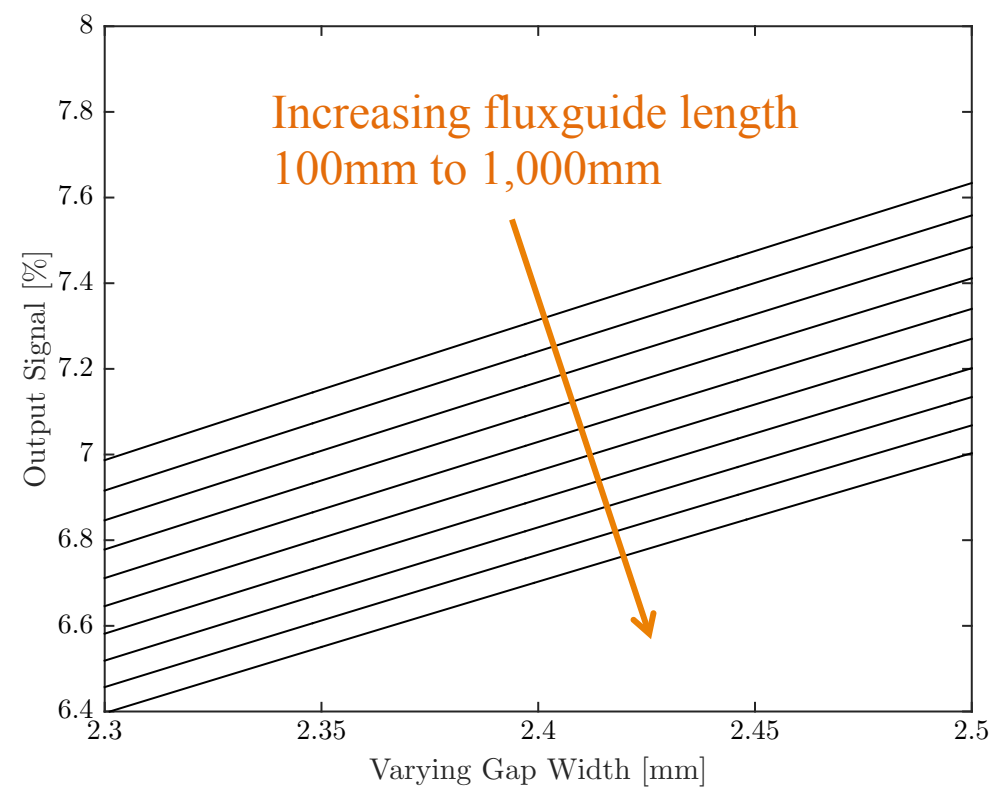

Figure 2.16: Percentage change in the output signal as a function of the varying gap width using different lengths for the flux guide assembly derived from the analytical model. 


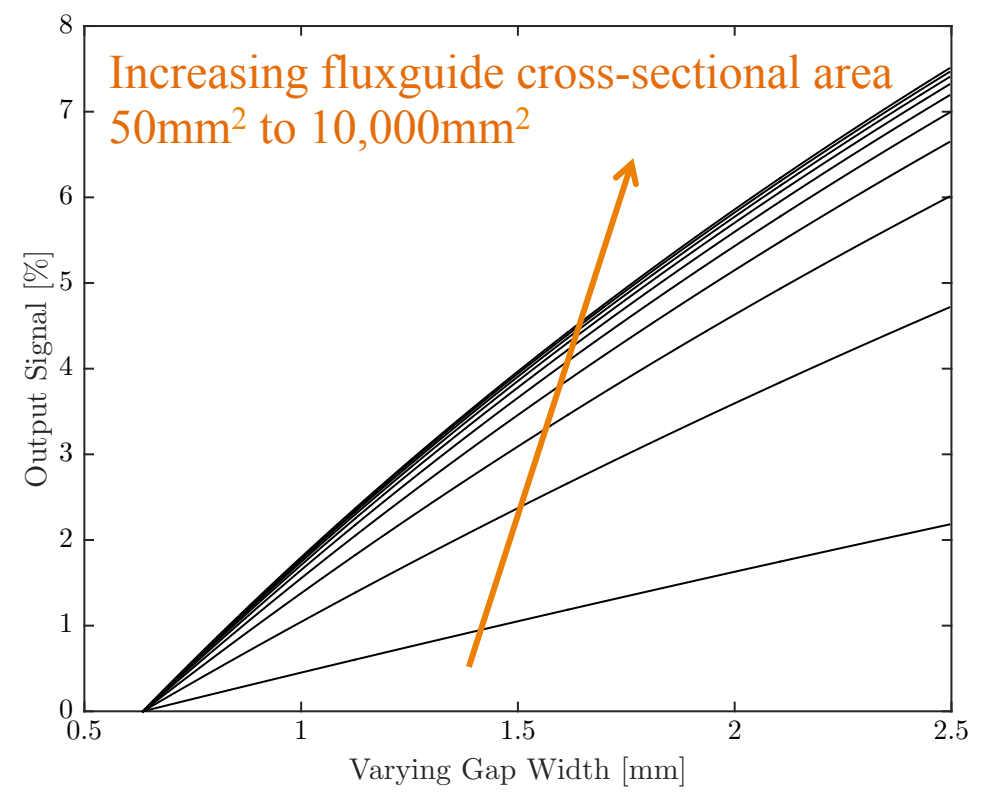

Figure 2.17: Percentage change in the output signal as a function of the varying gap width using various cross-sectional areas for the flux guide assembly derived from the analytical model.

\section{Geometry Selection}

The high-permeability M100 material used for the fabrication of the flux guide assembly is a highly brittle material and not easily machinable. For this reason, the design of the sensor should minimize any machining required to be done on the material to ensure the integrity of the material remains intact during the fabrication process. After going through different iterations for a clamping mechanism, a 'C' shaped geometry is selected with five legs to affix the sensor to the pump as shown in Figure 2.18. Each leg of the flux guide assembly can be a circular rod or a square block. Although the round edges of a cylindrical rod reduces material saturation at the sharp edges of the material, it is harder to machine and this makes the assembling process more complex. The square bars, on the other hand, can simply fit together in a form they are shipped and be bonded together without the need for any additional attaching mechanism. 


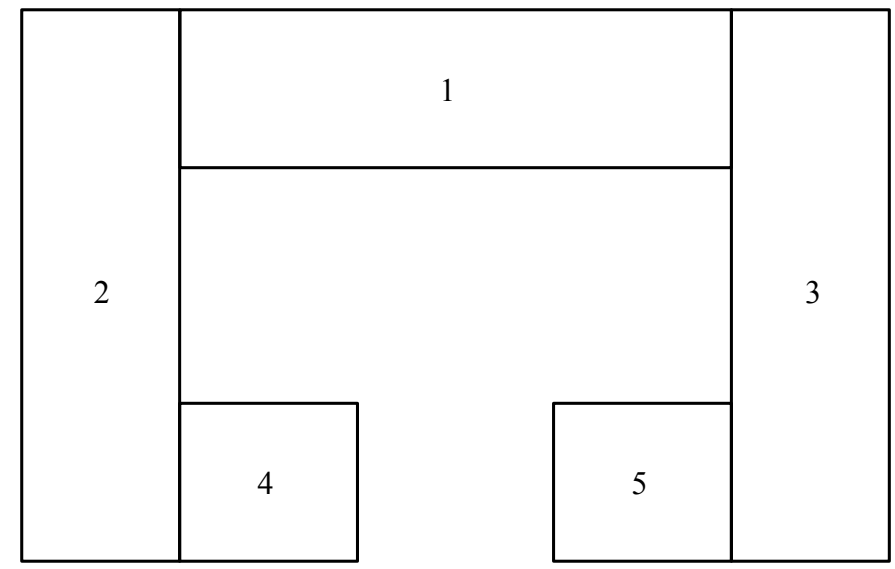

Figure 2.18: A 'C' Clamp design for the geometry of the flux guide assembly with the five legs numbered on the drawing.

For creating an adjustable clamping mechanism, there needs to be at least one degree of freedom in the flux guide design. Several design alternatives are tested both on paper and in the form of physical prototypes made from inexpensive low permeability material to test the feasibility and rigidity of the clamping mechanism once placed on the pump. The most ideal design based on the performance and machining minimization is a 5 legged ' $C$ ' clamp with legs 1 through 3 being square blocks bonded together, for example using epoxy, and legs 4 and 5 being circular rods passing through the legs 2 and 3 as shown in Figure 2.19. This design allows for adjustable clamping of the sensor on to the pump while minimizing the reluctance in the path of the magnetic flux going through the sensor.

To select the most ideal dimensions for the flux guide assembly, the sensitivity of the sensor was compared against the cost and material dimensions available from the vendor. Final dimensions of the flux guide assembly are shown in Figure 2.20 . 


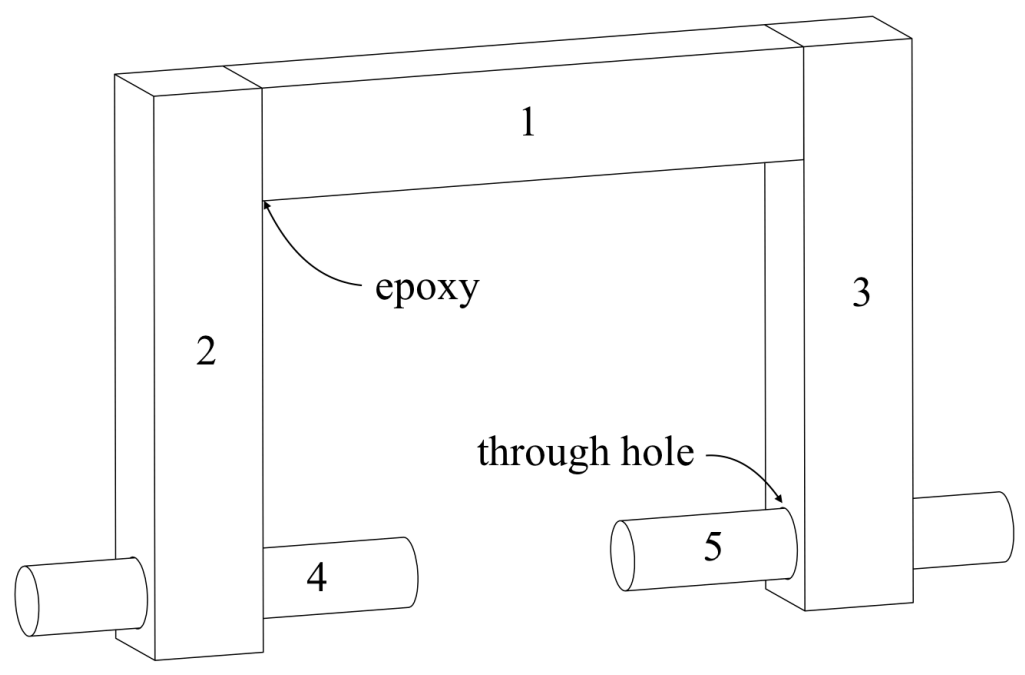

Figure 2.19: The isometric drawing of the flux guide clamping mechanism.

\subsubsection{Numerical Validation}

To numerically validate the effect of geometry on the output of the sensor, a more realistic model of the pump is developed using Comsol Multiphysics. The model includes all the components of the pump to scale with an exception of the impeller in which it assumes a straight impeller vane as opposed to the real curved impeller to simplify the geometry. The exploded view of the pump is shown in Figure 2.21.

One of the parameters of the design that needs to be tested is the material saturation. Although the use of the square block material is a more suitable option for the assembling of the flux guide, the sharp corners on the blocks will amplify the magnitude of the flux density that could cause flux saturation inside the material. Comsol Multiphysics is used to measure the intensity of the magnetic flux density at the sharp edges of the flux guide as shown in Appendix A. As the simulation result illustrates, there is no saturation occurring inside the material due to the low magnitude of flux flowing through the material. The maximum concentration of flux density detected inside the flux guide material is roughly $5 \mathrm{mT}$ and the saturation flux density of the M100 material is $420 \mathrm{mT}$. To further observe the flow of the flux inside the sensor, a $2 \mathrm{D}$ drawing of the sensor is plotted as shown in 


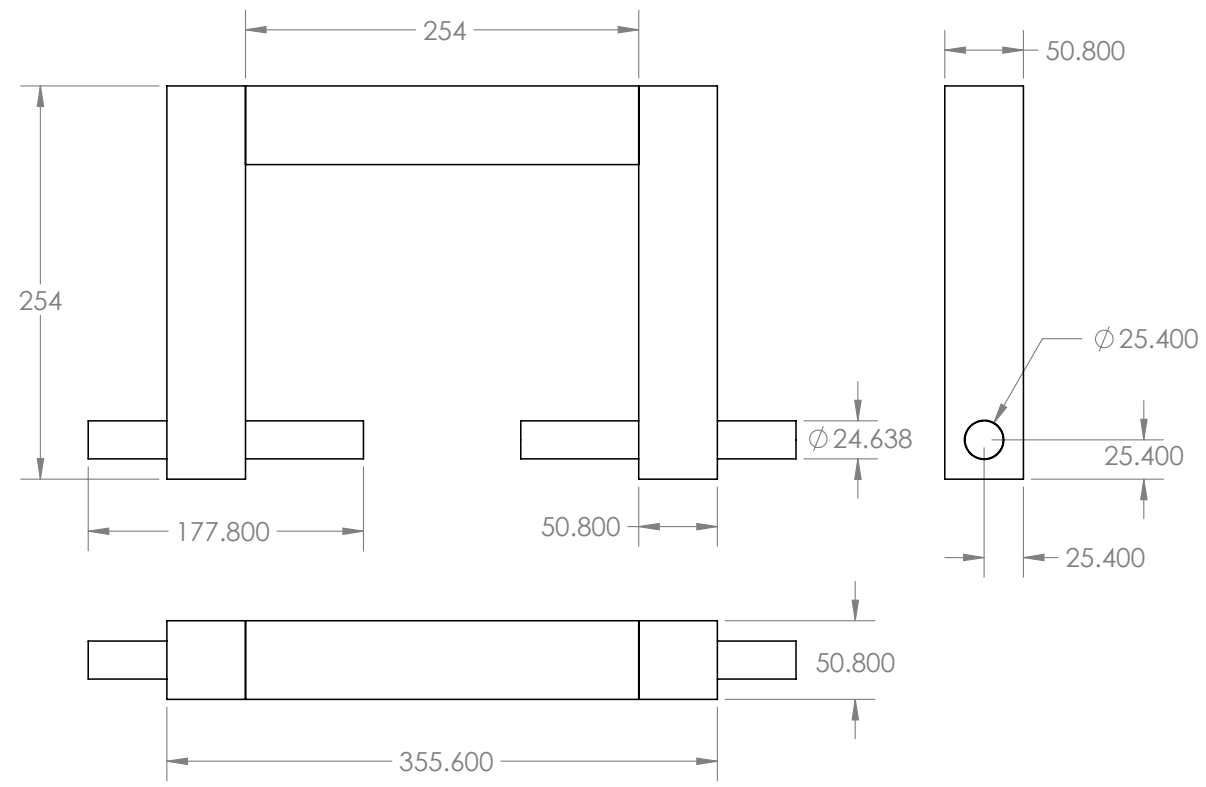

Figure 2.20: The dimensions of the flux guide assembly in [mm].

Figure 2.22, with the arrows indicating the direction of the magnetic flux density passing through the senor and the pump. It can be noticed from the plot that the majority of the flux field passes through the varying gap inside the pump and only a small portion of the field leaks through the housing and the pump's shaft.

After finalizing the geometry of the sensor, the more realistic Comsol model of the pump and the sensor assembly is used to estimate the change in the inductance of the magnetic coil as a function of the varying gap width. The simulation results are shown in Figure 2.23. As the figure indicates, the inductance of the coil drops $3.6 \%$ as the varying gap increases from $0.65 \mathrm{~mm}$ to $2.50 \mathrm{~mm}$. The difference in the results compared to the simple model of the sensor and the pump (Figure 2.12) is primarily due to the simplifying assumptions made in the flux guide geometry and also the flux leakage to the surrounding air and the pump housing that is increased in the more realistic model. 


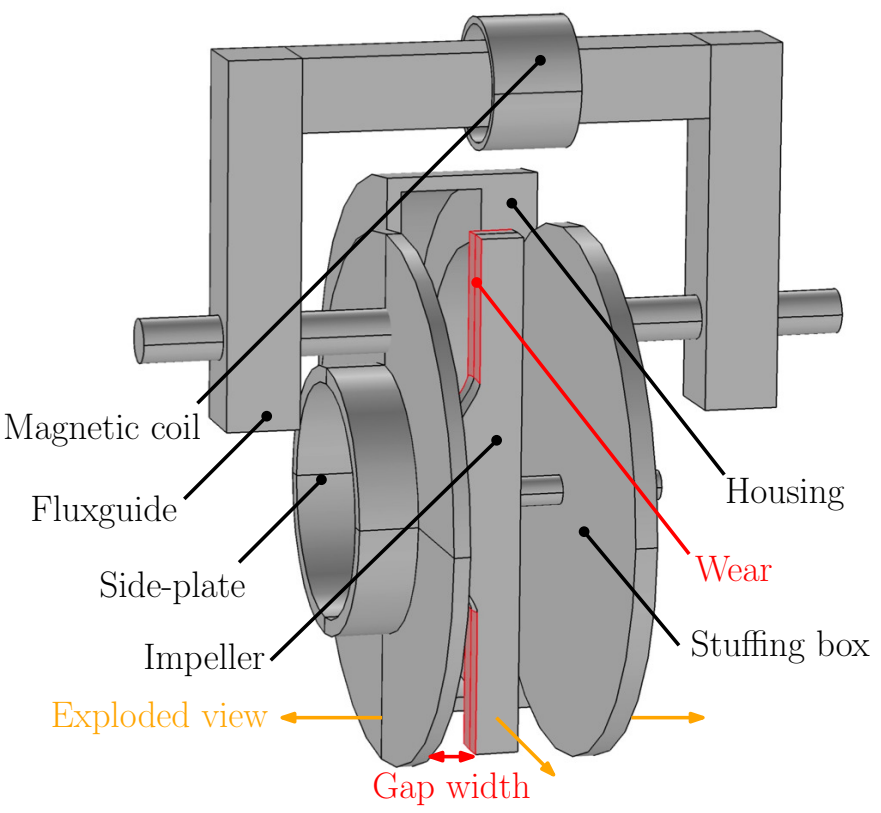

Figure 2.21: Exploded view of the more realistic pump model and the sensor created in Comsol Multiphysics. All the components of the pump and the sensor are labeled on the figure.

\subsection{Sensor Instrumentation}

Another important element of the wear sensor is the instrumentation and circuitry required to operate the sensor. The main component of the instrumentation is the magnetic coil placed on the flux guide assembly to drive flux through the pump and to evaluate the total reluctance of the magnetic circuit. To collect measurements, the sensor requires a portable instrumentation box that connects to the magnetic coil for continuous data logging while the pump is in operation. The instrumentation box includes a circuit for measuring the coil's inductance value, and a data acquisition unit to collect the readings and to transfer them to a computer for the user to analyze the data. This section will go through the design stages of the magnetic coil and the instrumentation box used in the magnetic wear sensor. 


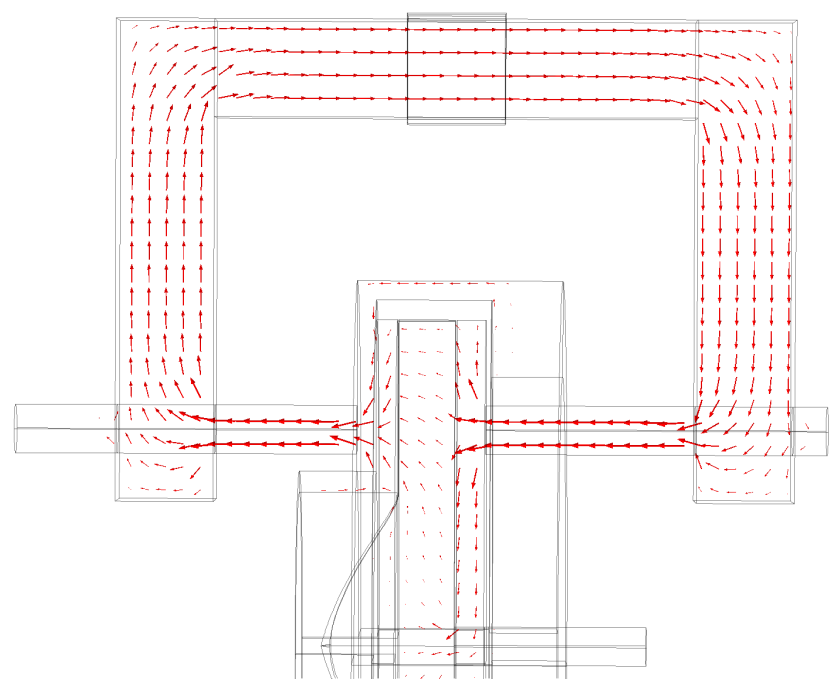

Figure 2.22: Path of magnetic flux inside the sensor and the flux guide assembly.

\subsubsection{Coil Design}

The use of a magnetic coil in the magnetic wear sensor has two purposes: to generate and drive magnetic flux through the sensor, and to monitor the reluctance change inside the path of the magnetic circuit. For this reason, the design of the magnetic coil plays a critical role in the performance of the sensor. The most important design parameters for the magnetic coil are its wire rating, number of turns, winding layers, coil dimensions, and bobbin geometry.

\section{Wiring Material}

The selection of the coil wire depends on the operating conditions of the sensor. To ensure that the wire is capable of carrying the current required to operate the sensor at a given frequency, the extreme operating conditions are identified to be $100 \mathrm{~mA}$ at a maximum $200 \mathrm{kHz}$ frequency. Based on these parameters, an American Wire 


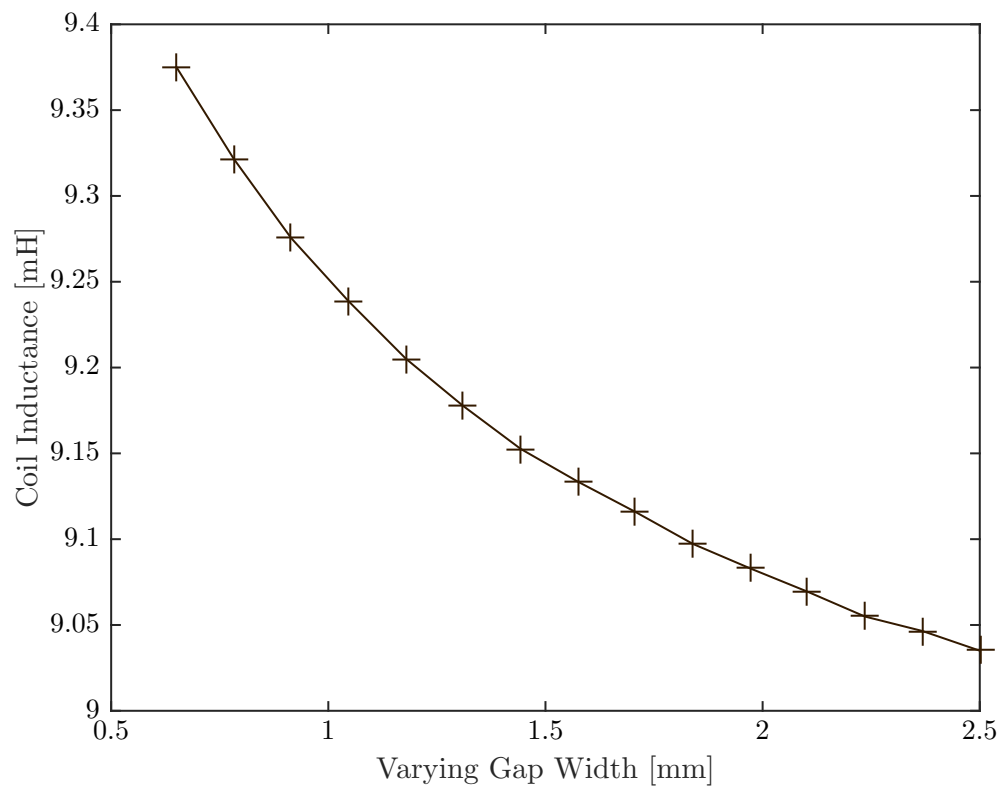

Figure 2.23: Comsol simulation results yielding the magnetic coil inductance as a function of the varying gap width for the more realistic model of the sensor and the pump. The coil is excited using a constant current source of $10 \mathrm{~mA}$ at $100 \mathrm{~Hz}$.

Gage 30 (AWG30) magnetic wire with enamel coating from EIS Incorporation, Atlanta, USA, is selected for the design of the magnetic coil. The parameter ratings of this wire are represented in Table 2.7.

Table 2.7: Properties of the American Wire Gape AWG30.

\begin{tabular}{cc}
\hline Property & Value [20] \\
\hline Diameter & $0.254 \mathrm{~mm}$ \\
Resistivity & $338.5 \Omega / \mathrm{km}$ \\
Maximum amperage & $0.142 \mathrm{~A}$ \\
Maximum frequency & $270 \mathrm{kHz}$ \\
\hline
\end{tabular}




\section{Coil Layout}

To find the most appropriate coil layout for the operation of the sensor, the change in the inductance of the coil is analyzed as a function of the varying gap width while alternating the total number of turns on the coil. After detail analysis of the results, it can be concluded that the total number of windings has no effect on the sensitivity of the sensor. The higher number of turns will only lead to a larger flux density value which if exceeds the material saturation flux density can lower the sensitivity of the sensor. One important parameter that should be considered is the parasitic capacitance of the coil. If the parasitic capacitance causes a resonance in the circuit at a resonating frequency below the operating point, the sensor can completely loose its sensitivity to the reluctance change. To estimate the maximum parasitic capacitance allowable in the coil, the equation of the resonating frequency is used. The resonating frequency in this case

$$
f_{\text {res }}=\frac{1}{2 \pi \sqrt{L_{\text {coil }} C_{\text {par }}}}
$$

can be written as a function of the coil inductance $L_{c o i l}$ and the parasitic capacitance between the coil windings $C_{\text {par }}$. The excitation frequency of the coil for the proposed sensor is $70 \mathrm{~Hz}$ and the reasoning behind this selection will be described in Chapter 3. If the maximum inductance of the coil is assumed to be $9.375 \mathrm{mH}$ based on the simulation results, by using Equation 2.24 the maximum allowable parasitic capacitance is calculated to be $551 \mu \mathrm{F}$.

To estimate the parasitic capacitance based on the number of windings and winding layers, an analytical model of the parasitic capacitance is used [21]. According to the analytical model, the total parasitic capacitance of a coil

$$
\begin{aligned}
C_{p a r} \cong 1.366 C_{t t} & \text { Singlelayer } \\
C_{p a r} \cong 1.618 C_{t t} & \text { Twolayer } \\
C_{p a r} \cong 1.830 C_{t t} & \text { Threelayer }
\end{aligned}
$$


depends on the number of coil layers and is written as a function of the turn-toturn capacitance of the coil wires for any number of turns $\mathrm{N}$ greater than 10 . The turn-to-turn capacitance

$$
C_{t t}=\varepsilon_{0} l_{t}\left[\frac{\varepsilon_{r} \theta^{*}}{\ln \frac{D_{o}}{D_{c}}}+\cot \left(\frac{\theta^{*}}{2}\right)-\cot \left(\frac{\pi}{12}\right)\right]
$$

is a function of the wire parameters, coil geometry and $\theta^{*}$, where $\theta^{*}$ is given by

$$
\theta^{*}=\arccos \left(1-\frac{\ln \frac{D_{o}}{D_{c}}}{\varepsilon_{r}}\right)
$$

and all of the parameters required to solve the above equations are labeled in Table 2.8. By solving Equation 2.25, Equation 2.26, Equation 2.27, Equation 2.28, and Equation 2.29, the parasitic capacitance of the coil is estimated to be $26.3 \mathrm{pF}$, $31.1 \mathrm{pF}$, and $35.2 \mathrm{pF}$, for the single layer, two layer and the three layer coils respectively. Since the values of the paracitic capacitance between the coil windings are very small, it can be concluded that the parasitic capacitance inside the coil can be neglected. Considering the number of turns on the coil also has a minor effect on the sensitivity of the sensor, a single layer 150-turn coil is selected to further simplify the fabrication process.

Table 2.8: Parameters for the parasitic capacitance analytical equation.

\begin{tabular}{ccc}
\hline Parameter & Definition & Value \\
\hline$\varepsilon_{0}$ & Absolute permittivity & $8.85 \times 10^{-12} \mathrm{~F} / \mathrm{m}$ \\
$\varepsilon_{r}$ & Relative permittivity of wire insulation (enamel) & 3.68 \\
$D_{o}$ & Wire outer diameter & $0.280 \mathrm{~mm}$ \\
$D_{c}$ & Wire core diameter (no insulation) & $0.254 \mathrm{~mm}$ \\
$D_{t}$ & Coil diameter & $50.8 \mathrm{~mm}$ \\
$l_{t}$ & Length of one turn $\left(\pi D_{t}\right)$ & $160 \mathrm{~mm}$ \\
\hline
\end{tabular}




\section{Coil \& Bobbin Geometry}

As seen from the many simulation models performed, the geometry of the coil has very little effect on the sensitivity of the sensor as long as the geometry is functional and the bobbin can be fitted on the sensor's flux guide legs. Since the flux guide is decided to have a square shaped cross-section, the coil's bobbin should also be square shaped to prevent any flux leakage. The length of the bobbin should be designed to fit the 150 turn coil in one layer. The design of the bobbin is presented in Figure 2.24 with the dimensions labeled on the drawing.
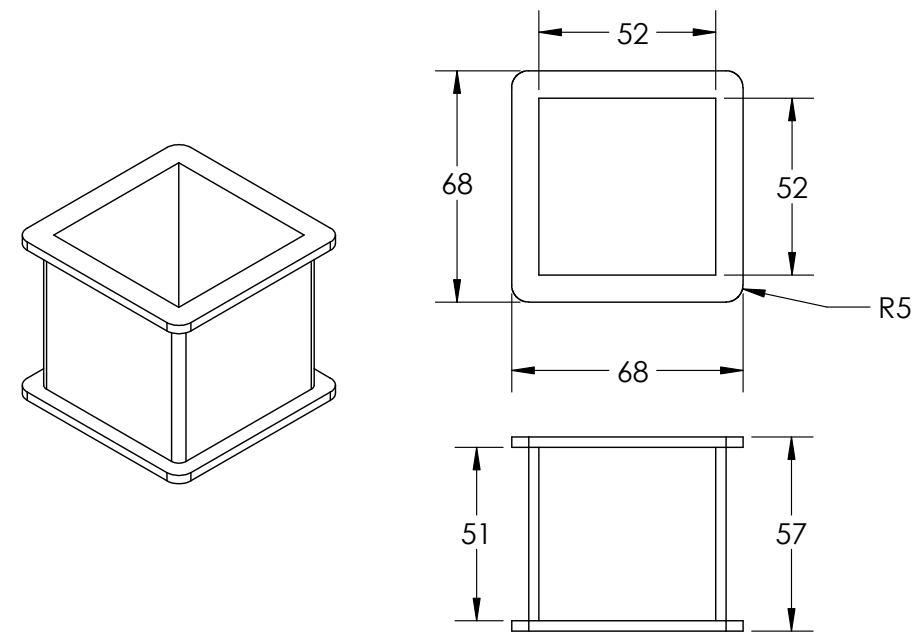

Figure 2.24: The design of the bobbin for the magnetic coil to be placed on the flux guide assembly with the dimensions in [mm].

\subsubsection{Inductance Measurement}

For the operation of the wear sensor, the designed magnetic coil is used to drive flux through the sensor. More importantly, the same coil is used to measure the change in the reluctance of the sensor caused by wear on the impeller blades of a centrifugal pump. As discussed in Section 2.2, the inductance of a magnetic coil is inversely proportional to the reluctance of its core material. To monitor wear, the inductance of the coil needs to be measured while the pump is in operation. 
To achieve this, several different methods are considered that allow for live inductance measurement. The most simple yet completely functional method for this sensor is through the use of an RL circuit created by connecting a resistor in series with the coil as shown in Figure 2.25. When an AC input voltage $V_{\text {in }}$ is applied across the circuit, the output voltage

$$
V_{\text {out }}=\frac{R}{R+j \omega L_{\text {coil }}} V_{\text {in }}
$$

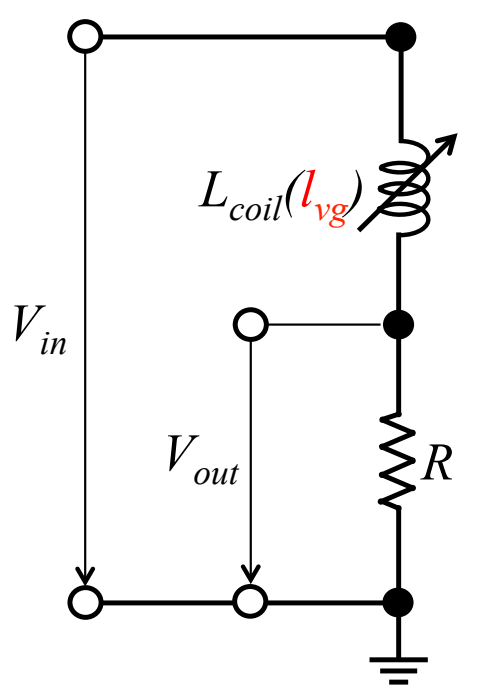

Figure 2.25: Simple RL circuit to measure the inductance of the magnetic coil.

measured across the resistor will be a function of the resistance $R$, the angular excitation frequency $\omega$, and most importantly the inductance of the coil $L_{c o i l}$. To further improve the analytical model of the electrical circuit, the parasitic capacitance of the coil $C_{\text {par }}$ and also the resistance of the coil wire $R_{\text {coil }}$ are also added to the simple RL circuit as shown in Figure 2.26. The equation for the output voltage

$$
V_{\text {out }}=\frac{R}{R+\left(R_{\text {coil }}+j \omega L_{\text {coil }}\right) \| \frac{1}{j \omega C_{\text {par }}}} V_{\text {in }}
$$

now includes all the circuit elements. To monitor the change in the magnitude of 


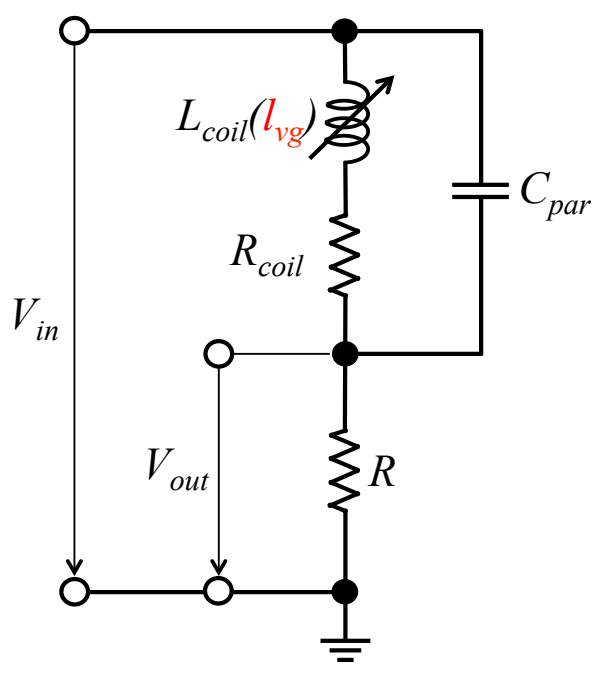

Figure 2.26: More realistic RLC circuit representing the coil connected in series with a resistor.

the output voltage as a function of the coil inductance, the magnitude of the ratio between the output voltage over the input voltage

$$
\left|\frac{V_{\text {out }}}{V_{\text {in }}}\right|=\sqrt{\frac{\left[R\left(1-\omega^{2} L_{\text {coil }} C_{\text {par }}\right)\right]^{2}+\omega^{2} R^{2} R_{\text {coil }}^{2} C_{\text {par }}^{2}}{\left[R_{\text {coil }}+R-\omega^{2} R L_{\text {coil }} C_{\text {par }}\right]^{2}+\left[\omega L+R R_{\text {coil }} \omega C_{\text {par }}\right]^{2}}}
$$

is derived by solving Equation 2.31 where the angular frequency, $\omega=2 \pi f$, can be written as a function of the excitation frequency $f$. The magnitude of the parasitic capacitance in this equation is known to be $26.3 \mathrm{pF}$ from the analytical model shown in Equation 2.25. The resistance of the coil wire can also be calculated by estimating the length of the wire and multiplying that value by the unit length resistivity of the AWG30 wire as noted in Table 2.7. Thus, the total resistance of the coil

$$
R_{\text {coil }}=150 \text { turn } \cdot 4 \frac{\text { edges }}{\text { turn }} \cdot 0.0508 \frac{m}{\text { edge }} \cdot 0.3385 \frac{\Omega}{m}
$$

is calculated to be $10.32 \Omega$. The only flexible design parameter in the circuit is the resistance value of the resistor $R$ that can be selected to maximize the change 
in the output signal as the inductance value varies from $9.375 \mathrm{mH}$ to $9.035 \mathrm{mH}$ at $100 \mathrm{~Hz}$ as predicted from the simulation results. This will result in the resistance being equal to $6 \Omega$. Next chapter will go into the details of optimizing all the circuit elements to maximize the sensitivity of the wear sensor. To observe the change in the output voltage as the inductance varies from no-wear to maximum-wear stages, the frequency response of the sensor is derived from the analytical model of the circuit and the resulting plots at the two extreme cases are shown in Figure 2.27. As it can be seen from the figure, if the sensor is excited using a $1.0 \mathrm{~V}$ AC signal at $100 \mathrm{~Hz}$, the output voltage changes from $647.1 \mathrm{mV}$ to $661.0 \mathrm{mV}$ as the gap varies from $0.65 \mathrm{~mm}$ to $2.50 \mathrm{~mm}$. This leads to a sensitivity of $7.51 \mathrm{mV} / \mathrm{mm}$.

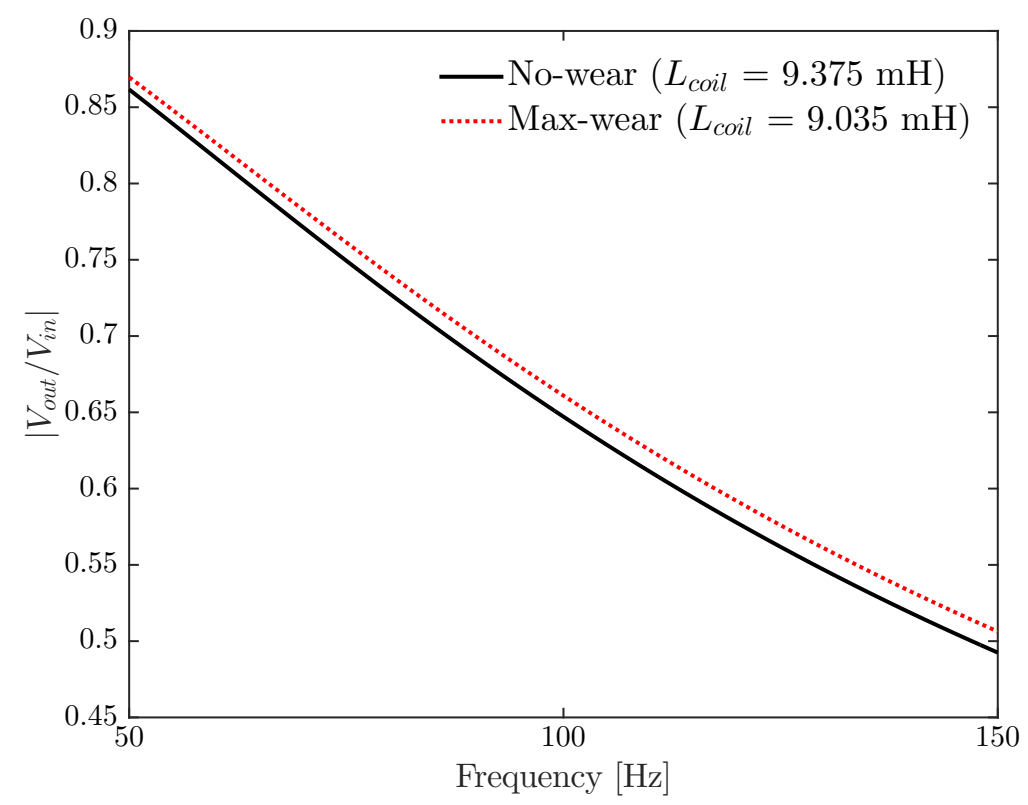

Figure 2.27: The change in the frequency response of the sensor as the magnitude of the varying gap increases from $0.65 \mathrm{~mm}$ to $2.50 \mathrm{~mm}$ derived from the analytical model of the sensor. 


\subsubsection{Circuit Design}

The sensitivity of the sensor can be enhanced by optimizing all the circuit elements used to measure the inductance of the coil. One of the parameters that can significantly improve the sensitivity of the sensor is the circuit's resonating frequency. As discussed in the beginning of this section, the presence of the parasitic capacitance inside the circuit creates resonance within the circuit that is undesirable if the operating frequency is selected to be above the resonating frequency. However, if the resonating frequency is manually tuned so that the sensor is operating just below the resonating frequency, the sensitivity of the sensor can be greatly improved. To accomplish this, an external capacitor $C_{e x t}$ is added in parallel with the coil and in combination with the parasitic capacitance $C_{p a r}$ a total capacitance of $C_{t o t}=C_{e x t}+C_{p a r}$ is created. The magnitude of the external capacitance is selected so that the resonating frequency $f_{\text {res }}$ falls at $105 \mathrm{~Hz}$ just above the $100 \mathrm{~Hz}$ operating frequency. For this, Equation 2.24 is used to calculate the total required capacitance

$$
C_{\text {tot }}=\frac{1}{4 \pi^{2} f_{\text {res }}^{2} L_{\text {coil }}}
$$

where the lowest magnitude of inductance is used to calculate for the resonating frequency. The equation yields the required external capacitance of $254 \mu \mathrm{F}$. By applying this value to the analytical model of the circuit, the frequency response of the sensor changes as shown in Figure 2.28. To evaluate the response of the sensor, the optimized resistance value $R$ used is calculated to be $54 \Omega$. As it can be seen from the modified sensor response, the magnitude of the output voltage changes from $481.5 \mathrm{mV}$ to $666.6 \mathrm{mV}$ with an input voltage signal of $1.0 \mathrm{~V}$ at $100 \mathrm{~Hz}$. This shows a sensitivity of $100.1 \mathrm{mV} / \mathrm{mm}$ which is more than $12 \times$ improvement over the previous model.

To excite the magnetic coil, a function generator with a constant power output is to be used with a sinusoidal voltage signal $V_{f g}$. To prevent the amplitude of the voltage to be dependent on the input impedance of the sensor, there needs to be a voltage buffer in place. For this reason, a voltage follower apparatus is designed and placed at the input terminal of the circuit. This ensures that the sen- 


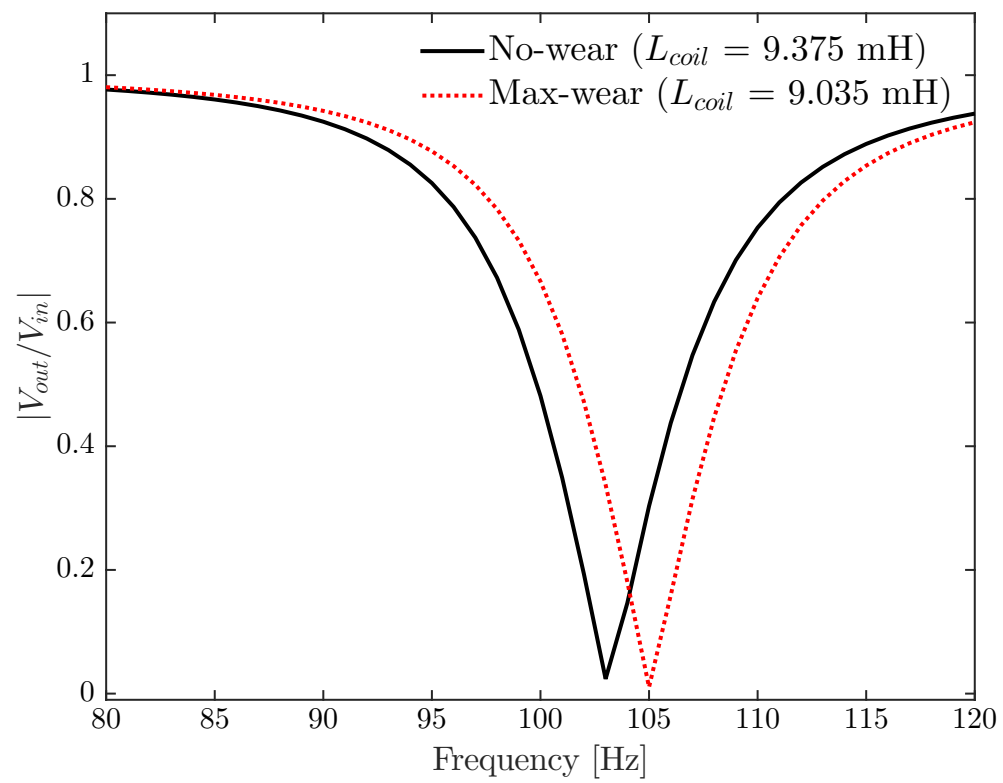

Figure 2.28: The change in the frequency response of the sensor as the magnitude of the varying gap increases from $0.65 \mathrm{~mm}$ to $2.50 \mathrm{~mm}$ with the addition of the external capacitance $C_{\text {ext }}$ to the circuit. The plot is generated from the analytical model of the sensor.

sitivity of the sensor is unchanged when the impedance of the circuit is varied due to the change in the inductance of the coil. The voltage follower circuit uses an Op-Amp (TL084CN) selected from STMicroelectronics Corporation in Geneva, Switzerland. The Op-Amp is powered using a DC voltage supply of $\pm 15 \mathrm{~V}$. The complete drawing of the circuit including the voltage follower is shown in Figure 2.29

\subsection{Data Analysis}

Up to now, the analysis of the wear sensor and its components are covered assuming the impeller blade is held stationary in between the probes of the sensor. The designed sensor, however, is expected to acquire and interpret sensor readings 


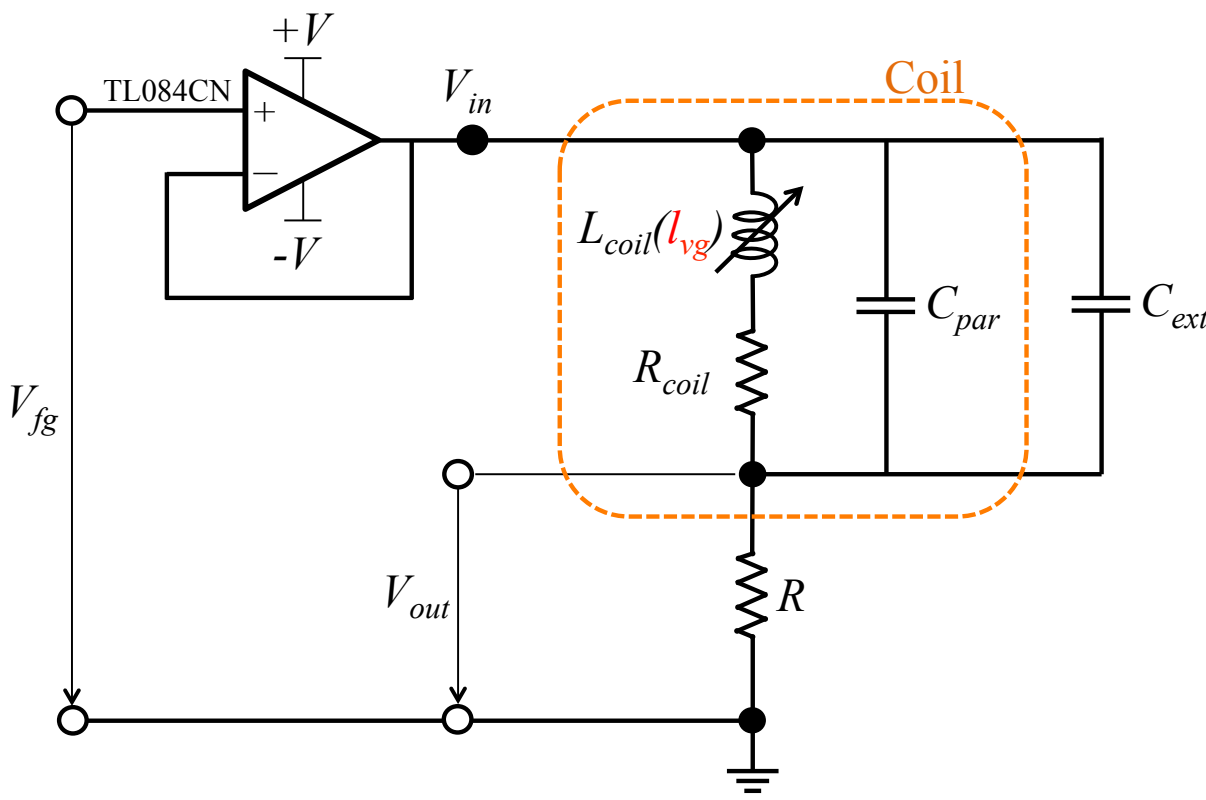

Figure 2.29: The complete drawing of the sensor's circuit including the voltage follower circuit.

while the impeller is moving. In this section, the output of the sensor is analyzed with the pump in operation and a data analysis method is proposed to accurately measure the gap width between the impeller and the side plate.

\subsubsection{Expected Sensor Response}

The output of the sensor is proportional to the change in the inductance of the magnetic coil placed on the flux guide assembly. The inductance can then be correlated to wear due to the increase in the varying gap between the impeller and the side plate. To observe the change in the inductance of the coil as the impeller does a full rotation, Comsol Multiphysics is used to numerically simulate the inductance as a function of the impeller's angular position for the two extreme cases of nowear and maximum-wear gap sizes. The resulting plots are shown in Figure 2.30. The peak inductance in these plots refer to the angular position when the impeller is crossing the probes of the sensor and therefore minimizing the gap inside the 
path of the magnetic circuit. The two peaks seen on the plot correspond to the two blades of the pump impeller. To correlate wear to the change in the inductance value of the magnetic coil, only the peak inductance value is taken into consideration.

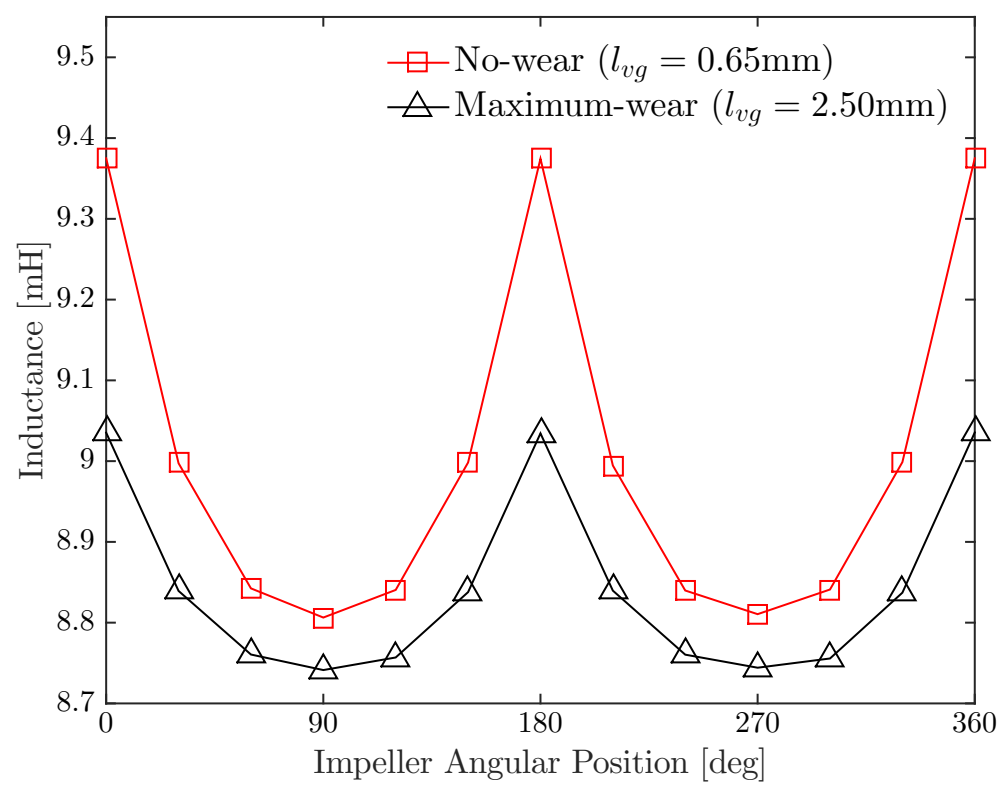

Figure 2.30: Inductance of the magnetic coil as a function of the impeller's angular position for both the no-wear and the maximum-wear cases derived from Comsol simulations.

To detect the impeller blades as they cross the probes of the sensor, the magnetic coil needs to be excited at a minimum frequency of $10 \mathrm{kHz}$. The issue with high excitation frequency is the presence of eddy currents. Since the housing of the pump is made from cast iron with an electrical conductivity of roughly $1 \times 10^{6} \mathrm{~S} / \mathrm{m}$, high frequency magnetic field will induce eddy currents to form inside the pump material, completely diminishing the sensitivity of the sensor. For this reason, Comsol Multiphysics is used to estimate the maximum excitation frequency possible without inducing strong eddy currents inside the pump's housing. The finite element simulation results suggest the ideal frequency to be below 
$100 \mathrm{~Hz}$. Although by operating at $100 \mathrm{~Hz}$ the effect of eddy current on the output of the sensor is negligible, the excitation frequency will not be fast enough to acquire useful readings while the pump is in operation. To overcome this problem, the signal can be over sampled and the effect of wear is determined on the shape of the output signal.

To ensure that there are sufficient readings while the impeller is crossing the probes of the sensor, the sampling frequency is calculated with respect to the angular speed of the impeller blades. The maximum typical rotational speed of an open impeller centrifugal pump inside a plant environment is around 3600 RPM. To analytically calculate the time it takes the impeller blade to cross the probes of the sensor, the equations of the arc length and the tip velocity of the impeller are used to derive an equation for the sensor passing time

$$
t_{\text {passing }}=\frac{l_{\text {probe }}}{\omega_{\text {pump }} r_{\text {imp }}}
$$

as a function of the sensor probe length $l_{\text {probe }}$, pump rotational speed $\omega_{\text {pump }}$, and the radius of the pump's impeller $r_{\text {imp }}$. The probe length is known to be roughly $0.030 \mathrm{~m}$, and the impeller's maximum rotational speed is converted into $376.99 \mathrm{rad} / \mathrm{s}$ with a radius of $0.1778 \mathrm{~m}$. By using these values, the sensor's passing time is calculated to be $0.4476 \mathrm{~ms}$ for each impeller blade. To acquire accurate measurement while the impeller is passing the probes of the sensor, it is safe to assume that the sampling frequency needs to be such that there are at least twenty readings while the impeller is positioned inside the path of the sensor. This indicates that the sampling period needs to be limited to $0.02238 \mathrm{~ms}$ which translates into a minimum sampling frequency of $44 \mathrm{kHz}$. To achieve the best performance from the sensor, a sampling frequency of $500 \mathrm{kHz}$ is used to acquire wear measurements. The selection of this sampling frequency is further explained in the following chapter of this thesis. 


\subsubsection{Data Analysis Method}

To measure the width of the varying gap while the impeller blade is passing the probes of the sensor, the coil is excited at $70 \mathrm{~Hz}$ and the reasoning behind the selection of this frequency is explained in Chapter 3. The data is then sampled at $500 \mathrm{~Hz}$ and the output time signal is transformed into the frequency domain using the fast Fourier transform algorithm (FFT) in MATLAB. Assuming the impeller is rotating at $900 \mathrm{RPM}$, which is the typical average speed of centrifugal pumps inside a plant, a generic time signal is created and transformed into the frequency domain as shown in Figure 2.31. As indicated on the drawing, there are three peaks expected on the FFT plot. The first peak is detected at $15 \mathrm{~Hz}$ and it corresponds to the impeller rotational speed of 900 RPM. The second peak is at $30 \mathrm{~Hz}$ referring to the impeller blade passing frequency. Since there are two blades of the impeller of the pump, the passing frequency is double the frequency of the impeller's rotational speed. The last peak is at $70 \mathrm{~Hz}$ that is the excitation frequency of the coil. To measure the change in the inductance of the coil due to the change in the varying gap width between the impeller and the side plate, the amplitude of the FFT plot at $15 \mathrm{~Hz}$ and $30 \mathrm{~Hz}$ are monitored. A scatter plot of the amplitude at $15 \mathrm{~Hz}$ versus the amplitude at $30 \mathrm{~Hz}$ can be used to indicate the change in the varying gap width.

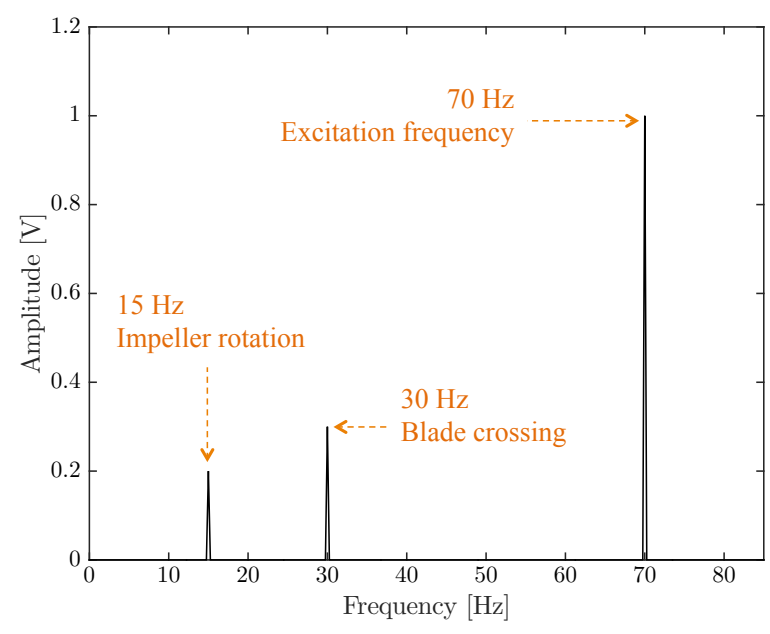

Figure 2.31: Expected FFT response of the generic time signal from the sensor while the pump is operating at 900 RPM. 


\section{Chapter 3}

\section{Experimental Validation}

This chapter will start by explaining the design and fabrication process of the sensor prototype as described in Chapter 2. The rest of the chapter will focus on sensor characterization, calibration process, experimental setup, and finally the experimental results and analysis.

\subsection{Sensor Prototype}

To experimentally validate the functionality of the sensor, a physical prototype needs to be built and tested while installed on a centrifugal pump inside a plant environment. The sensor prototype includes three individual components, namely, the flux guide, the magnetic coil, and the portable instrumentation box for collecting sensor readings.

\subsubsection{Flux Guide Assembly}

\section{Material Acquisition}

The flux guide assembly is made from the high-permeability M100 material as described in Section 2.3. For the assembling of the flux guide geometry, three square blocks with a length of $254 \mathrm{~mm}$ and a side width of $50.8 \mathrm{~mm}$, and two cylindrical rods with a diameter of $24.6 \mathrm{~mm}$ and a length of $177.8 \mathrm{~mm}$ are purchased from the 
National Magnetics Group Incorporation. The tolerance of the materials purchased are $\pm 0.5 \mathrm{~mm}$ rated by the manufacturer. Since the selected material is highly brittle, the machining done on the material needs to be minimized. for this reason, the three square blocks are to be bonded together while the two cylindrical rods need to go through the square blocks to complete the magnetic circuit as shown in Figure 2.19.

\section{Machining of the Flux Guide Assembly}

To accommodate for the two cylindrical rods inside the square blocks, two through holes with a diameter of $25.40 \mathrm{~mm}$ are punctured inside the blocks. For the machining of the holes, a water jet cutter is used with extra-low pressure settings desirable for the highly-brittle materials. The water jet cutter uses pressurized water mixed with an abrasive substance such as sand to puncture the two holes through the M100 blocks. To dampen the impact of the water mixture on the surface of the material so to avoid the chipping of the surface, two thin aluminum plates with a thickness of $1.6 \mathrm{~mm}$ are attached on both sides of the blocks.

\section{Magnetic Epoxy}

To assemble the three square blocks together without performing additional machining on the material, the blocks can be bonded together in a form of a ' $\mathrm{C}$ ' shape bracket using epoxy. The downside of using an epoxy is that it will induce an inevitable gap inside the flux guide where the epoxy solution is applied. Since this gap will have a relatively high reluctance value, it will significantly reduce the sensitivity of the sensor. To minimize the reluctance of the gaps created, the epoxy is mixed with the high-permeability M100 sintered particles, acquired from the National Magnetics Group Incorporation, with a relative permeability of $\mu_{r}=10,000$ through a 50\% weight ratio to create a magnetic epoxy with a relatively higher permeability. The epoxy itself is selected based on the strength test results of several different brands when applied on magnetic core materials [22]. The selected epoxy is Loctite Adhesive Cartridge, E-00NS Epoxy manufactured by Henkel Corpora- 
tion from Dusseldorf, Germany, purchased from McMaster Carr. The picture of the epoxy and the sintered particles is shown in Figure 3.1. The mixture is then applied to the blocks and the final assembly is left at room temperature for 24 hours to cure. The final geometry is shown in Figure 3.2 To protect the flux guide assembly from fracturing, a water-resistant super-cushioning polyurethane foam with a thickness of $6.35 \mathrm{~mm}$ is bonded to the sensor as shown in Figure 3.3

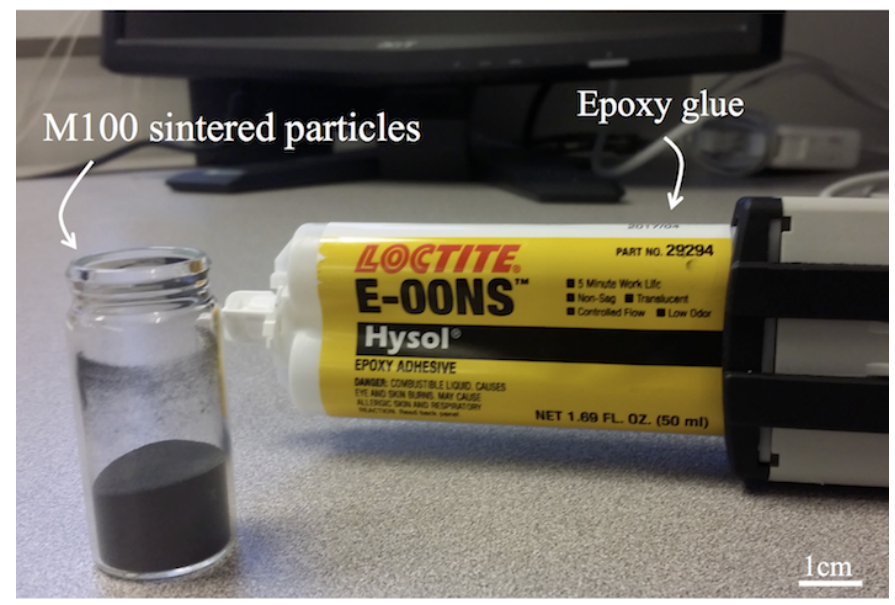

Figure 3.1: The epoxy used to attach the flux guide components with the sintered M100 particles acquired from National Magnetics Group.

\subsubsection{Magnetic Coil}

For the fabrication of the magnetic coil and the bobbin, the design parameters obtained in Section 2.4 are used. The bobbin is 3D printed with the geometry modeled in SolidWorks as shown in Figure 3.4. Since the current flowing through the wire is no more than $1 \mathrm{~mA}$ and the sensor takes merely few seconds to acquire each reading, the effect of temperature on the bobbin material is neglected. The AWG30 magnetic wire used for the magnetic coil is purchased from McMaster Carr. To wind the wire around the bobbin, a manual coil winding apparatus is used that creates a rotating mechanism for the bobbin to be placed on and counts the total number of revolutions as the bobbin is turned. The winding apparatus was used from the research lab supervised by Dr. Martin Ordonez and with the help of 


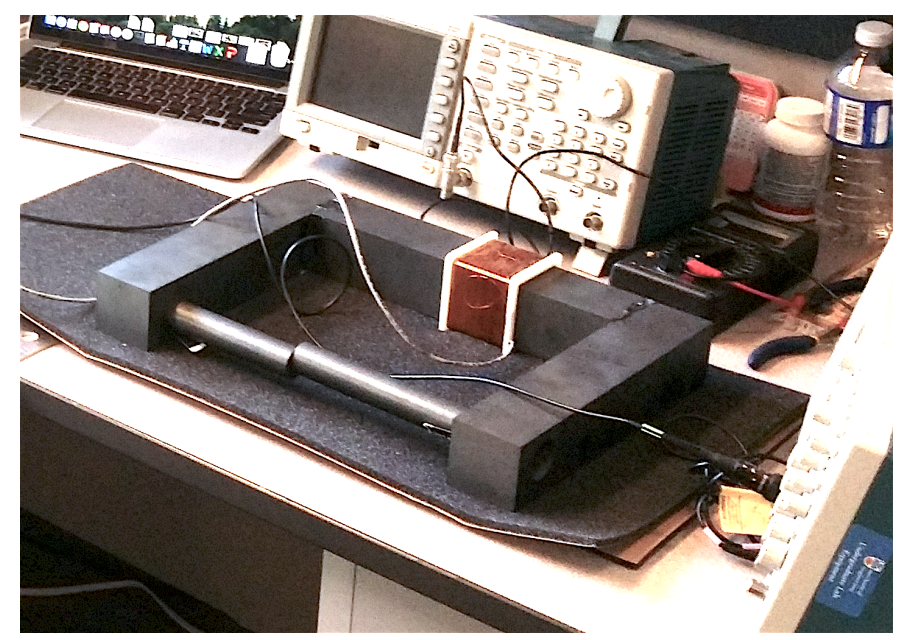

Figure 3.2: The fabricated flux guide assembly with the magnetic coil.

his $\mathrm{PhD}$ student Navid Shafiei. To protect the windings of the coil, a thin sheet of transparent adhesive tape is applied to the coil as shown in Figure 3.5.

\subsubsection{Instrumentation Box}

The portable instrumentation box is meant to connect the sensor to a computer device for data logging. The box is connected via a plug-in connection to the magnetic coil and it includes all the circuit components of the sensor including a data acquisition system to sample the data. For the exterior of the box, a sheet metal instrument case (LB-1525) is selected and purchased from Lee's Electronics in Vancouver, Canada. The connection from the box to the coil is made by using a two-wire shielded cable that is soldered on one end to the coil and a heat shrink is used to protect the connection. On the other end of the cable, a male circular DIN connector is attached that fits onto a female DIN connector mounted on the instrumentation box. The inductance measuring RLC circuit as represented in Figure 2.29 is made using a breadboard like circuit panel to allow for easy replacement of the circuit elements. The DAQ unit used for the sensor is an NI USB-6212 unit manufactured by National Instruments Corporation. The DAQ selected has a maximum sampling frequency of $500 \mathrm{kS} / \mathrm{s}$ that is sufficient for the operation of the sensor. The final design of the instrumentation box is shown in Figure 3.6. 


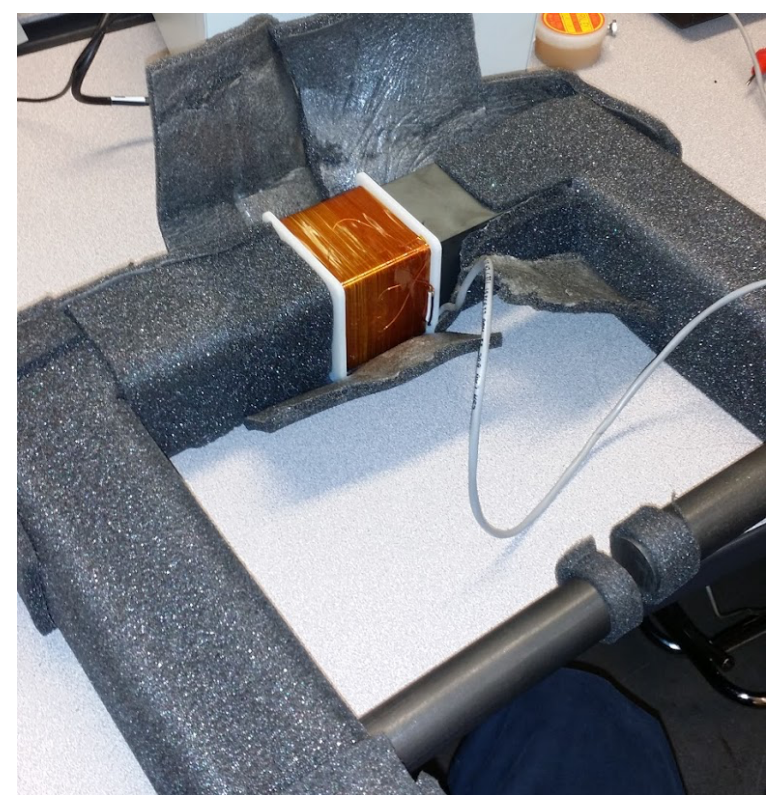

Figure 3.3: The flux guide assembly with the cushioning foam.

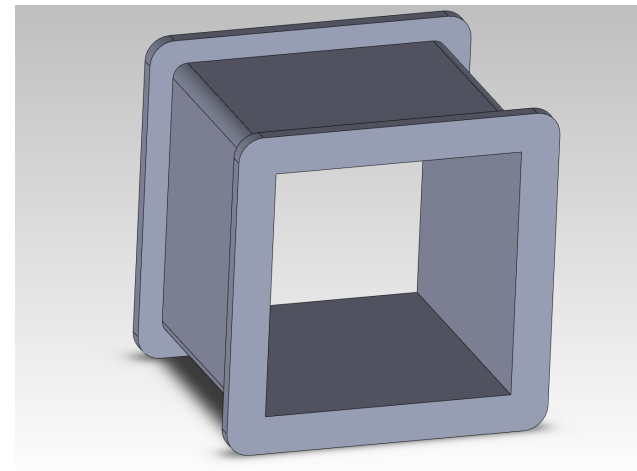

Figure 3.4: The design of the coil bobbin in SolidWorks.

The complete sensor prototype includes the flux guide assembly, the magnetic coil installed onto the upper leg of the flux guide, and the instrumentation box that connects the sensor to a computer running LabVIEW software as shown in Figure 3.7. The cost of the material and the components used for the fabrication of the sensor prototype are shown in Table 3.1. The cost of components that are 


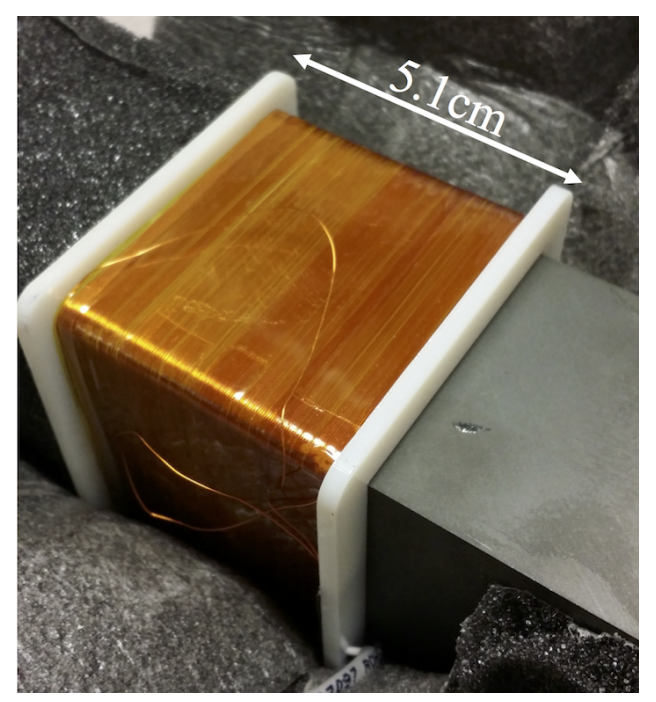

Figure 3.5: Prototype of the magnetic coil.

Table 3.1: Cost of the material and the components used for the fabrication of the physical prototype.

\begin{tabular}{ccc}
\hline Part & Quantity & Cost per unit (CAD) \\
\hline M100 square block & 3 & $\$ 650.00$ \\
M100 cylindrical rod & 2 & $\$ 165.00$ \\
NI Daq USB-6212 & 1 & $\$ 1,530.00$ \\
E-00NS Epoxy & 1 & $\$ 14.47$ \\
Cushioning foam (24” by 24”) & 1 & $\$ 35.23$ \\
Instrument box & 1 & $\$ 32.00$ \\
DIN connector & 2 & $\$ 17.74$ \\
Shielded cable $(1 \mathrm{~m})$ & 2 & $\$ 19.38$ \\
\hline
\end{tabular}

below $\$ 10.00$ are excluded from the list.

\subsection{Sensor Characterization}

Once the physical prototype of the sensor is built, the performance of the sensor and its components need to be characterized and verified with the analytical model prior to running experiments on the pump. 


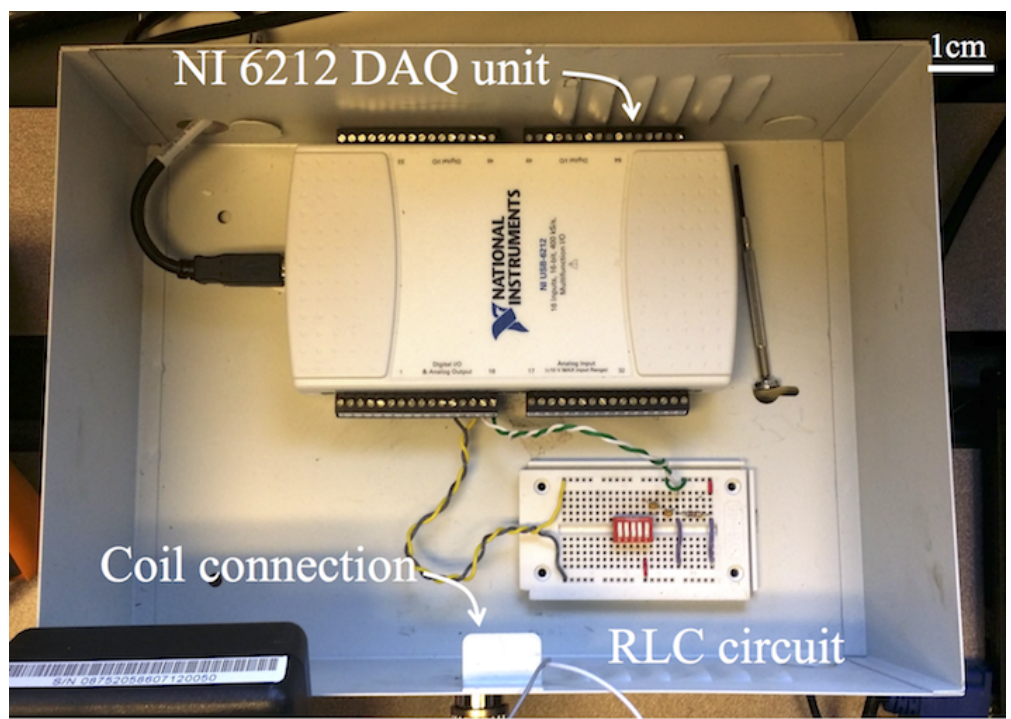

Figure 3.6: The instrumentation box with the electrical circuit and the DAQ unit. The box also includes a pivoting door (not shown in the picture) to protect the instruments inside.

\subsubsection{Coil Inductance and Parasitic Capacitance}

Without attaching the external capacitor to the measurement circuit, the frequency response of the sensor is acquired to calculate the coil's inductance value and the parasitic capacitance between the windings of the coil. To acquire the frequency response, a function generator is connected to the input terminals of the sensor and the peak-to-peak amplitude is set to $1.0 \mathrm{~V}$ while the frequency is varied from $500 \mathrm{~Hz}$ to $200 \mathrm{kHz}$. To measure both the inductance $L_{\text {coil }}$ and the parasitic capacitance $C_{\text {ext }}$ of the coil assembly from the frequency response of the sensor, initially only the lower frequency readings of the output signal are taken into consideration where the effect of the parasitic capacitance of the magnetic coil can be neglected. By assuming no parasitic capacitance, the magnitude of the output voltage over the input voltage 


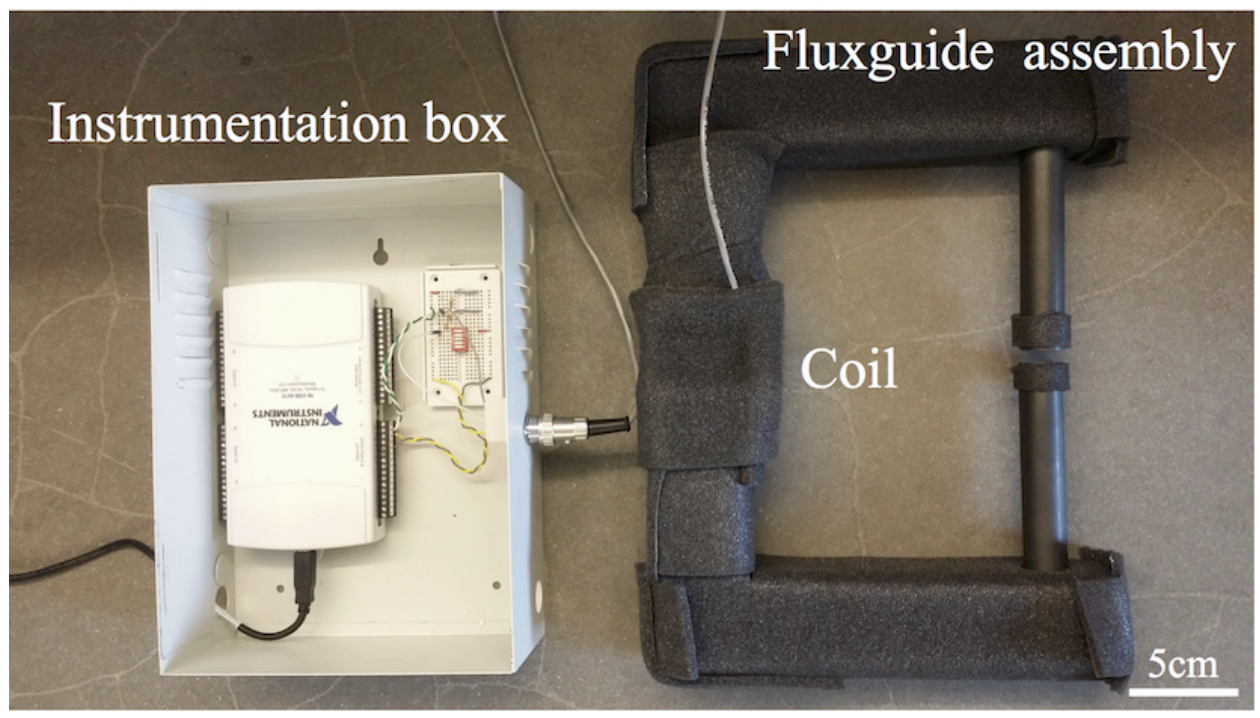

Figure 3.7: Picture of the complete sensor prototype including the flux guide assembly, the coil, and the instrumentation box.

$$
\left|\frac{V_{\text {out }}}{V_{\text {in }}}\right|=\frac{R}{\sqrt{R^{2}+\left(2 \pi f L_{\text {coil }}\right)^{2}}}
$$

is written merely as a function of the known resistance $R$ and the coil's inductance $L_{\text {coil }}$. The analytical model of the simplified sensor can be used to derive an equation for the inductance of the coil

$$
L_{\text {coil }}=\frac{R}{2 \pi f} \sqrt{\left(\frac{V_{\text {in }}}{V_{\text {out }}}\right)^{2}-1}
$$

as a function of the input voltage $V_{i n}$, output voltage $V_{\text {out }}$, frequency $f$, and the resistance $R$. Through the means of a least square estimation, the inductance of the magnetic coil placed on the flux guide is estimated to be $33.1 \mathrm{mH}$. The value of the measured inductance is in the same order of magnitude as the inductance value predicted from the analytical results $\left(L_{\text {coil }}=9.375 \mathrm{mH}\right)$ as shown in Section 2.3 . The higher inductance value observed in the physical measurement is primarily due to the circuit being fully closed while the measurements are taken with the probes of the sensor in contact with each other. The magnitude of the inductance 
is also verified through the use of an RLC-meter (BK-Precision 875B) to ensure the analytical model matches the physical prototype. The plot of the experimental measurements and the least-square estimation is shown in Figure 3.8.

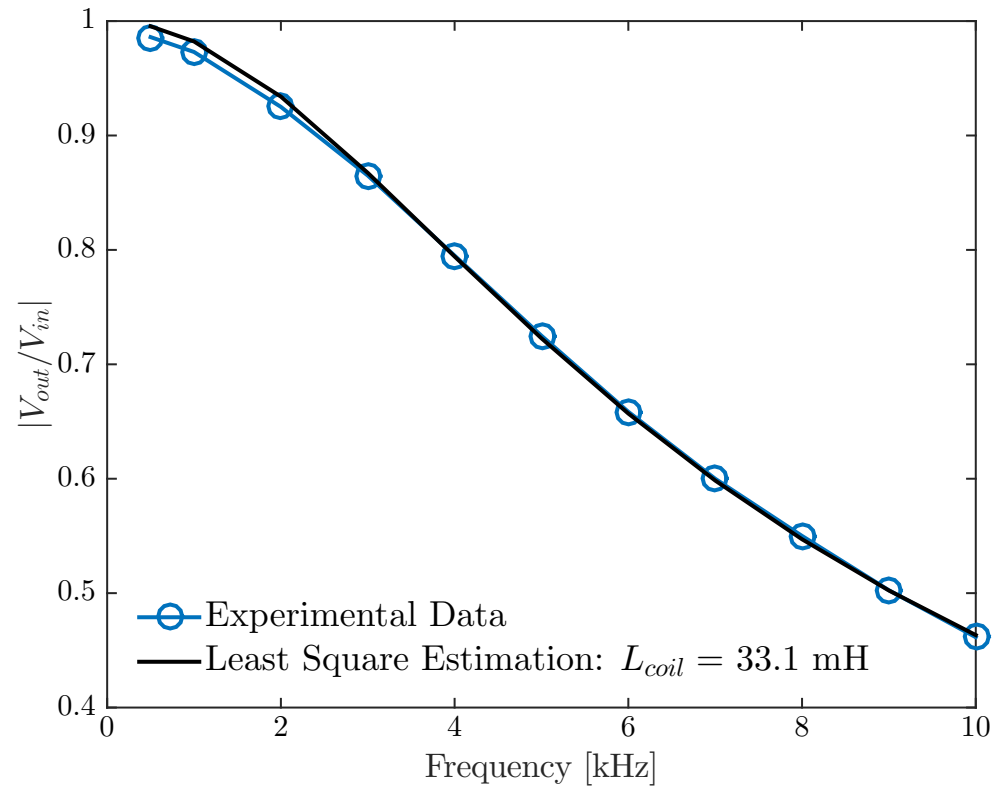

Figure 3.8: Frequency response of the sensor: experimental measurements and the least square estimation.

The resonating frequency of the circuit is estimated to be roughly $160 \mathrm{kHz}$. By using the equation for the resonance frequency as stated in Equation 2.24, the parasitic capacitance of the coil is calculated to be $29.8 \mathrm{pF}$. This is very close to the analytical prediction $\left(C_{p a r}=26.3 \mathrm{pF}\right)$ as noted in Section 2.4 . To ensure that neglecting the capacitance did not alter the inductance calculation shown in Equation 3.2, the impedance of the inductor, $Z_{L}=j \omega L_{\text {coil }}$, is compared against the impedance of the parasitic capacitor, $Z_{C}=\frac{1}{j \omega C_{e x t}}$, at the cutoff frequency. The cut-off frequency is increased incrementally at each iteration to bring the impedance of the inductor at least 100 times higher than the impedance of the capacitor $L_{c o i l} \times 100 \leq \frac{1}{\omega^{2} C_{e x t}}$ for the inductance measurement. This brings the cutoff frequency to $10 \mathrm{kHz}$. 


\subsubsection{Circuit Optimization}

Once the physical parameters of the coil are identified, all the external circuit elements such as the resistance $R$, the external capacitance $C_{e x t}$ and also the excitation frequency $f$ need to be optimized. Based on the simulation results, the excitation frequency of the coil needs to be kept below $100 \mathrm{~Hz}$ to avoid eddy currents being formed inside the pump material. To adjust the resonating frequency of the circuit to $100 \mathrm{~Hz}$, a capacitor with a capacitance of $C_{e x t}=100 \mu \mathrm{F}$ is selected. To optimize the rest of the circuit parameters, the output voltage of the sensor is modeled with the new capacitance value using the analytical model shown in Equation 2.32. By comparing the value of $R$ with the change in the output voltage obtained from the analytical model, the optimum resistance value is estimated to be $7.5 \Omega$ as shown in Figure 3.9. The same analytical model is used to compare the change in the output voltage as a function of the excitation frequency. Based on the analytical results shown in Figure 3.10, the ideal operating frequency is estimated to be $70 \mathrm{~Hz}$.

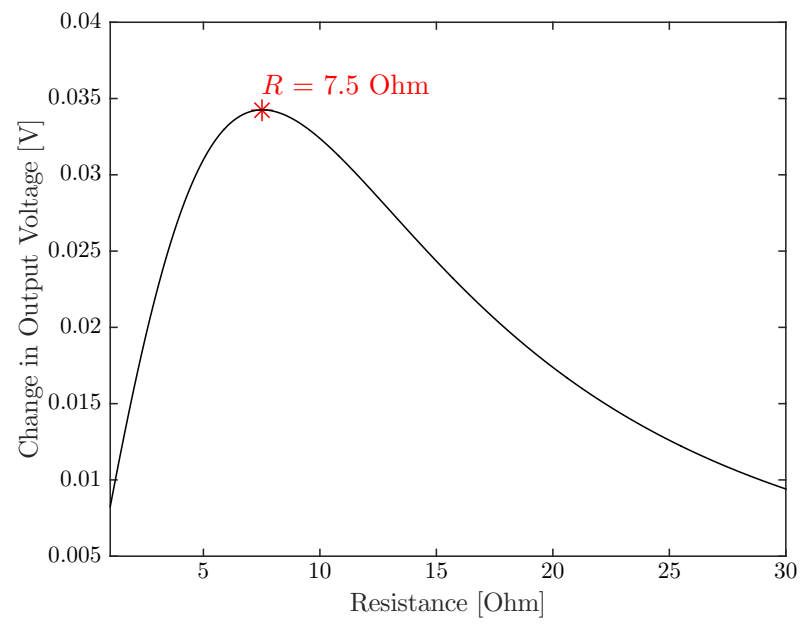

Figure 3.9: Maximizing the change in the output signal as a function of the resistance value of $R$. 


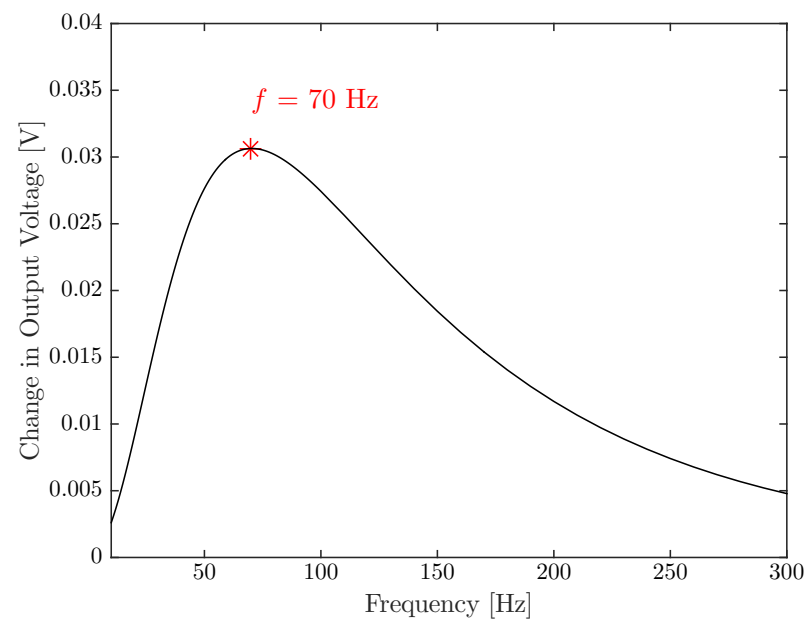

Figure 3.10: Maximizing the change in the output signal as a function of the excitation frequency $f$.

\subsubsection{Benchtop Experiments}

Prior to performing experiments on a physical pump, a benchtop experimental setup is created to test the behaviour of the sensor in a more controlled environment. By using a simpler setup, many of the uncontrollable parameters during the experiment can be prevented which could in turn help improve the result of any future experiments done on a physical pump. The benchtop experimental setup includes two parallel cast iron plates separated by thin plastic gaskets at known thicknesses. The gap between the two plates is increased incrementally while the sensor is placed perpendicular to the surface of the plates as shown in Figure 3.11. The frequency response of the sensor is collected at each gap width to observe the change in the output of the sensor as shown in Figure 3.12. As it can be observed, the frequency response of the sensor shifts to the right as the gap width between the two parallel plates increases. This is very compatible with the expected sensor response as shown in Figure 2.28. With these results, the experiments can be moved toward a real pump inside a plant. 


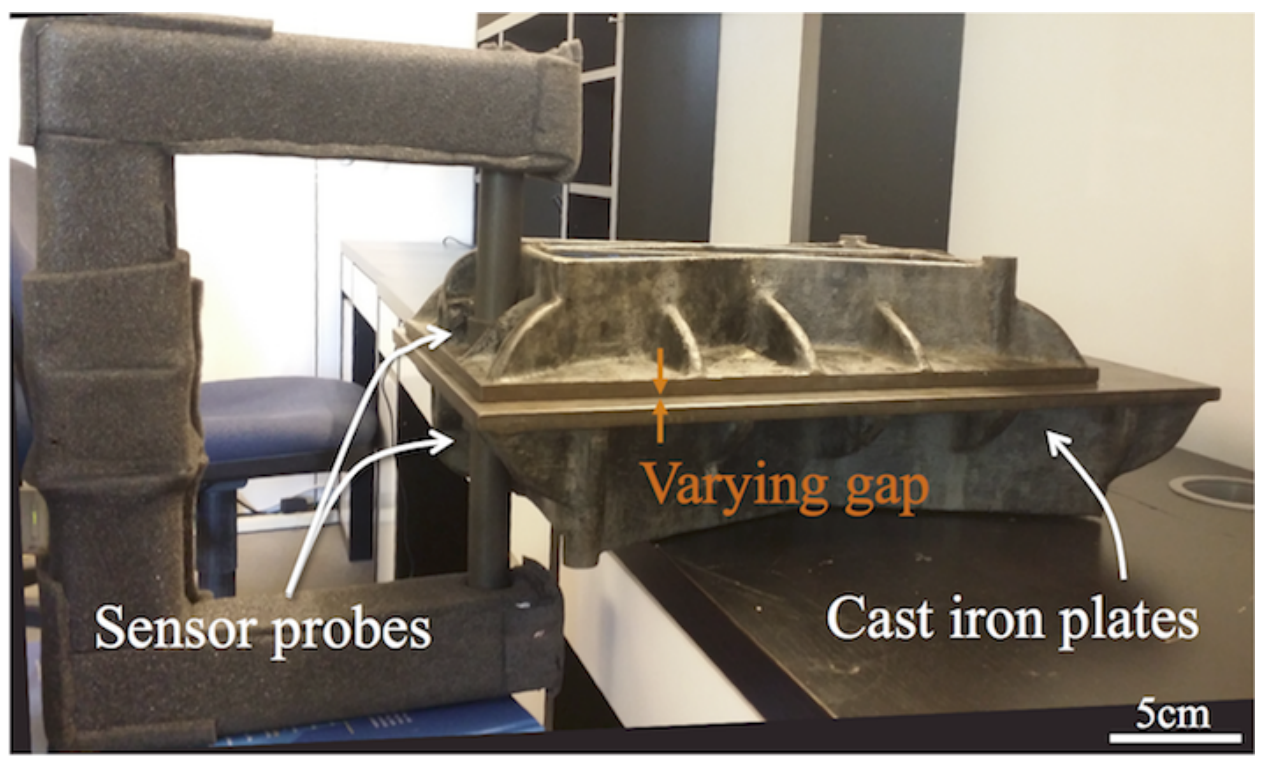

Figure 3.11: The benchtop experimental apparatus with the sensor placed perpendicularly to the two parallel cast iron plates.

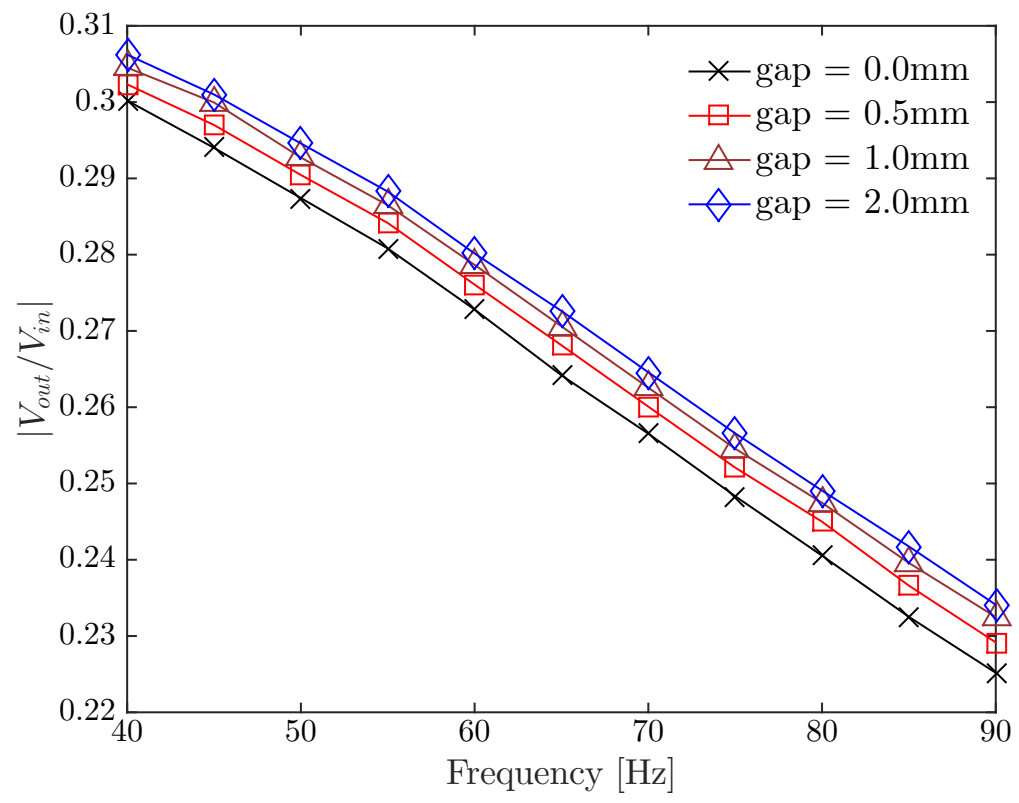

Figure 3.12: The frequency response of the sensor as the gap between the two parallel cast iron plates vary from $0.0 \mathrm{~mm}$ to $2.0 \mathrm{~mm}$. 


\subsection{Experimental Setup}

\subsubsection{Pump Loop}

After validating the analytical model of the sensor and testing its performance in the benchtop experiment, the sensor is to be tested on a physical pump. The pump used to perform the experiments in this project is a 40-hp two blade open impeller centrifugal pump manufactured by Westcan installed at the UBC's Pulp and Paper Center pilot plant. The pump is built entirely from cast iron with a relative magnetic permeability of $\mu_{r}=500$. The picture of the pump with the direction of the flow is shown in Figure 3.13 and the schematic diagram of the pump loop inside the facility is shown in Figure 3.14. The setup is used to pump water with pulp suspension through a double tank mixing loop. Since the water and the pulp consistency both have a magnetic permeability close to that of the vacuum, their presence has no effect on the output of the sensor.

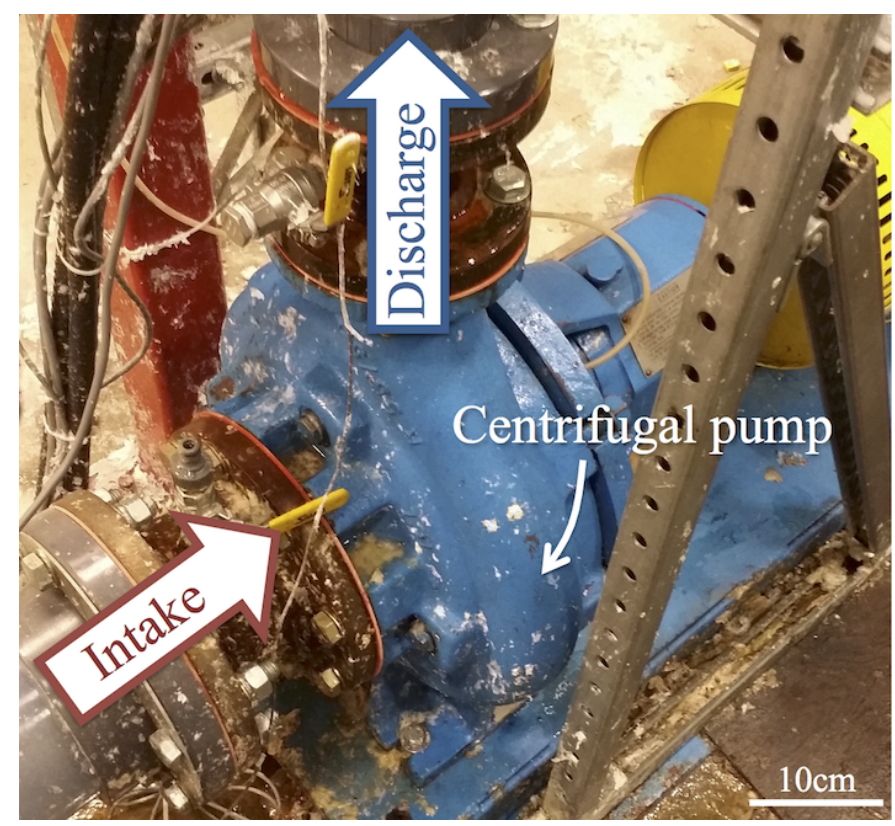

Figure 3.13: Westcan Centrifugal pump installed in the PPC Pilot Plant. Direction of the flow is labeled on the figure. 


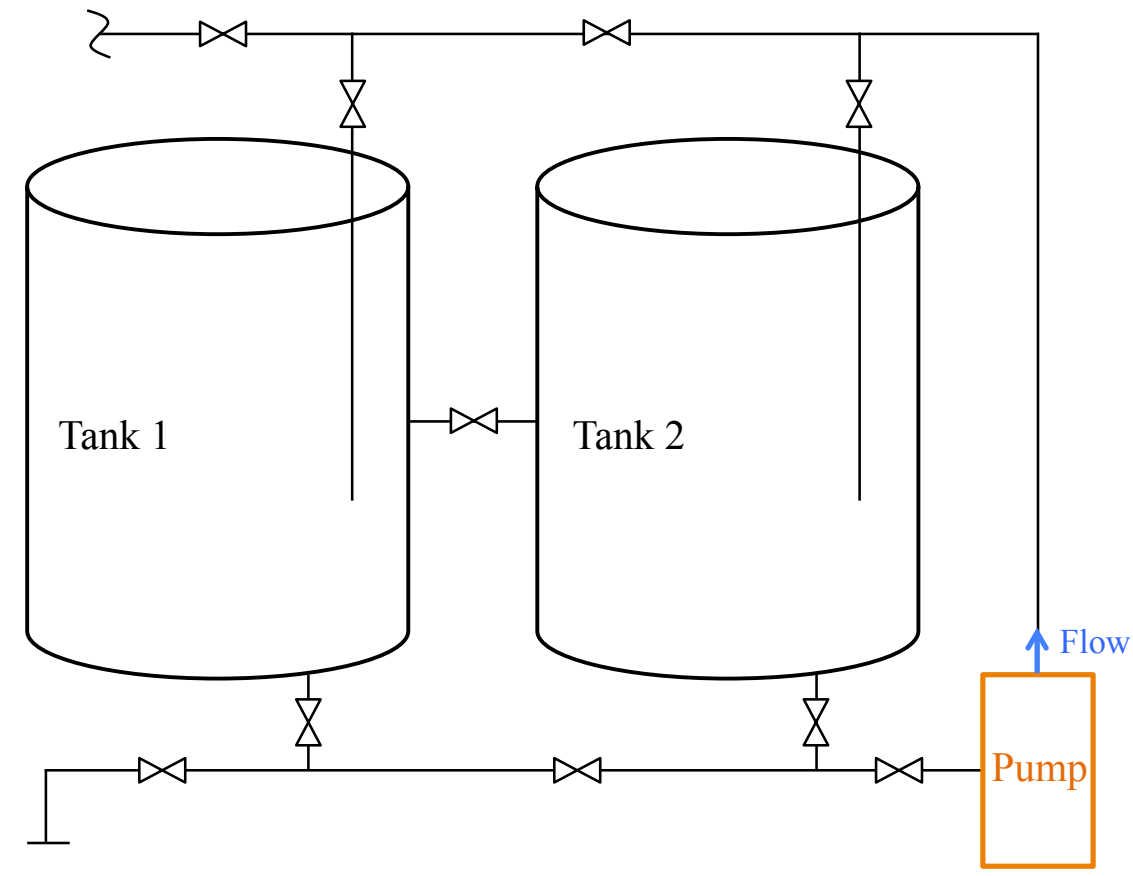

Figure 3.14: Schematic diagram of the pump loop in the Pulp and Paper Center at UBC.

\subsubsection{Pump Adjustments}

Through observing the manufacturer's data sheet of the pump and the pump's cross-sectional drawing, it is identified that there is a large void in between the side plate and the housing of the pump. The geometry and the dimensions of the void is acquired by placing a piece of clay material behind the pump's side plate at the location where the sensor is to be installed. After the clay material is removed, the void is verified to have a width close to $2 \mathrm{~cm}$ which is a large enough gap inside the path of the magnetic flux to completely diminish the functionality of the sensor on the pump. To fully close the path of the magnetic circuit inside the pump, the void needs to be filled with a material that has a relative permeability close to that of the pump material. To achieve this, a high permeability polymer is formed by mixing a two-part polyurethane kit by Smooth-Cast Onyx with the M100 sintered particles in a 50\% weight ratio. To further increase the permeability of the 
polymer, thin plates of the M100 material are cut and placed inside the mixture as shown in Figure 3.15. The magnetic polymer is then poured in the void and left at room temperature to cure. This problem is expected for all the pumps that utilize a side plate. For the majority of the centrifugal pumps, however, the sensor can be installed without any additional modifications.

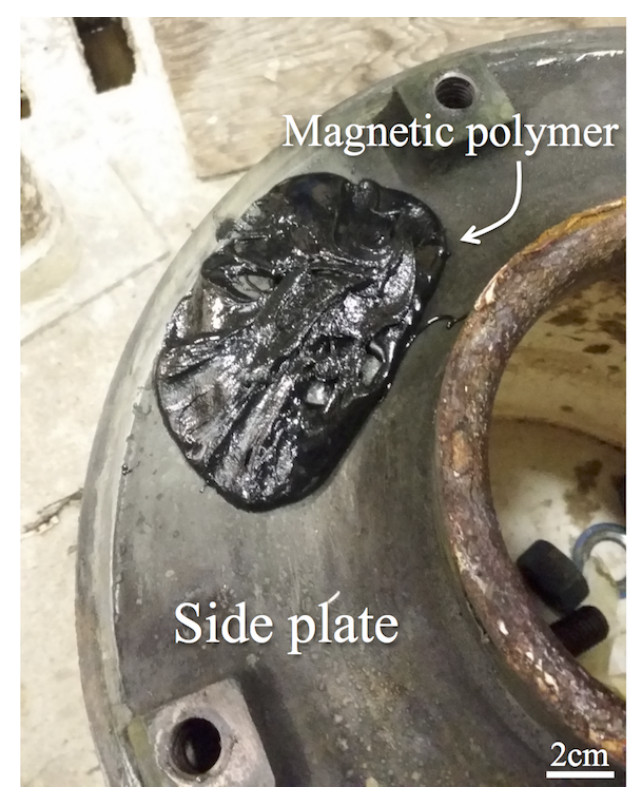

Figure 3.15: The magnetic polymer mixture poured on the pump's side plate.

\subsubsection{Sensor Installation}

After adjusting the pump to accommodate the sensor, the sensor is installed on the edge of the pump for data collection. To keep the sensor affixed to the pump and to protect the sensor from tipping over, the assembly is also supported from an above fixture by the means of two wire cables. The probes of the sensor are then clamped on the sides of the pump to close the magnetic circuit. The picture of the sensor installed on the pump is shown in Figure 3.16. The instrumentation box connected to the coil is placed on a table beside the pump and the box is connected to a computer for data acquisition. To power the op-amp in the circuit, a DC power 
supply is also attached to the instrumentation box and a function generator is used to excite the coil.

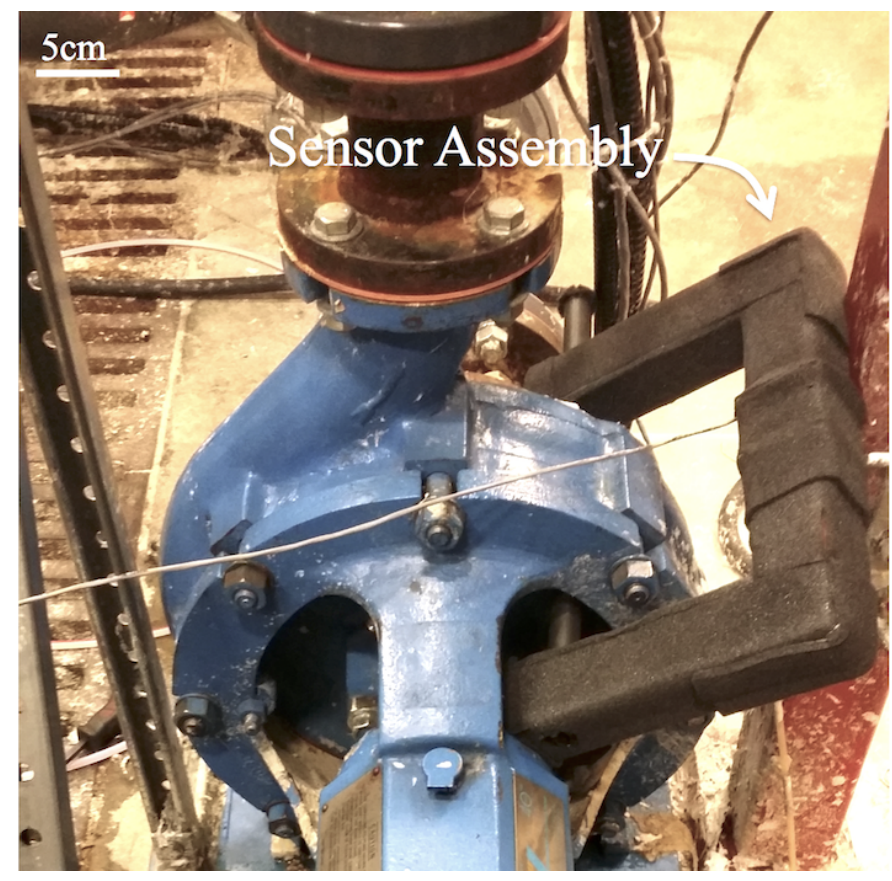

Figure 3.16: The sensor assembly installed on the pump.

\subsubsection{Sensor Calibration}

To calibrate the magnetic wear sensor and to observe the change in the output of the sensor as wear accumulates on the tip of the impeller blades of the centrifugal pump, the sensor needs to take measurements at various stages of the impeller's life while it is being eroded. Since wear occurs very slowly over a period of months or even years, an alternative method for calibrating the sensor is proposed. In this method, the gap between the impeller and the side plate is manually varied to reproduce the effect of wear on the varying gap width inside the pump. To manually vary the gap, thin ring gaskets with a thickness of $0.8 \mathrm{~mm}$ are inserted behind the pump housing to shift the stuffing box along with the impeller outwards. Since the gaskets are slightly compressed when placed inside the pump, the thickness of each gasket is estimated to be $0.75 \mathrm{~mm} \pm 0.05 \mathrm{~mm}$ through measuring the physical 
change in the location of the stuffing box with respect to the housing of the pump. The initial clearance inside the pump between the impeller and the side plate is set to $0.40 \mathrm{~mm} \pm 0.05 \mathrm{~mm}$. Gaskets are then placed one at a time and the process is repeated three times to increase the gap from an initial width of $0.40 \mathrm{~mm} \pm$ $0.05 \mathrm{~mm}$ to a width of $2.65 \mathrm{~mm} \pm 0.20 \mathrm{~mm}$. Each time a gasket is inserted, the sensor is installed on the pump and measurements are collected while the pump is running. The location of the gaskets on the schematic drawing of the pump is shown in Figure 3.17. The location is also labeled on the physical pump as shown in Figure 3.18

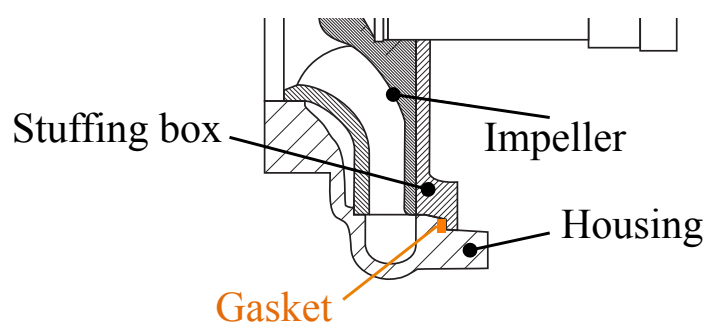

Figure 3.17: The location of the ring gaskets on the schematic cross-sectional view of the pump.

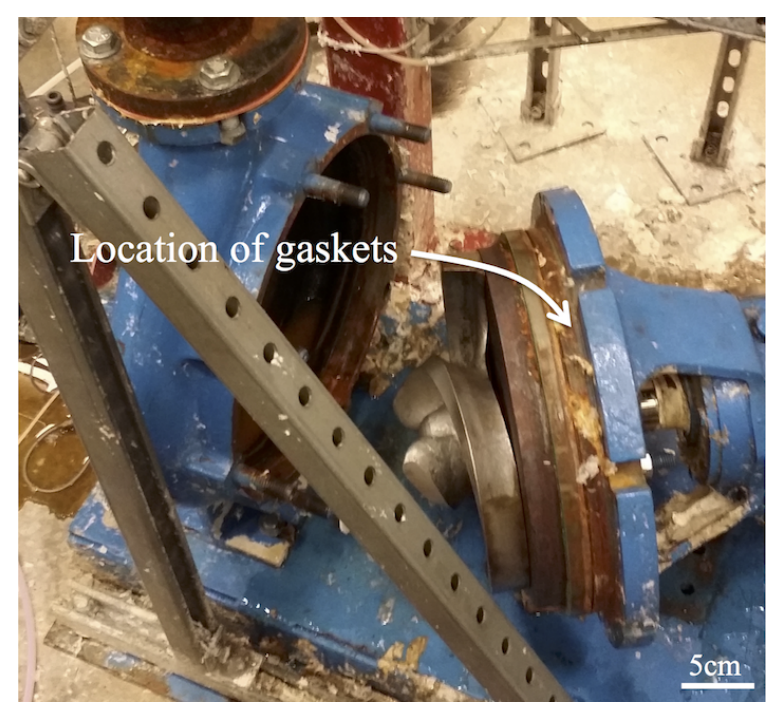

Figure 3.18: The location of the ring gaskets on the centrifugal pump. 


\subsection{Experimental Results \& Analysis}

\subsubsection{Sensor Output}

Once the sensor is installed, data is collected while the pump is held stationary to analyze the output voltage signal generated from the circuit. To obtain the measurements, the coil is excited at $70 \mathrm{~Hz}$ and data is collected at $50 \mathrm{kHz}$ sampling frequency for a duration of one second. The rotational speed of the pump is then increased to 900 RPM using a variable frequency drive (VFD) connected to the motor that runs the pump. Data is again collected with the same sampling conditions and the output of the sensor is plotted against time to observe the change in the behaviour of the sensor. The output voltage signal for both pump rotational speeds of 0 RPM and 900 RPM are presented in Figure 3.19
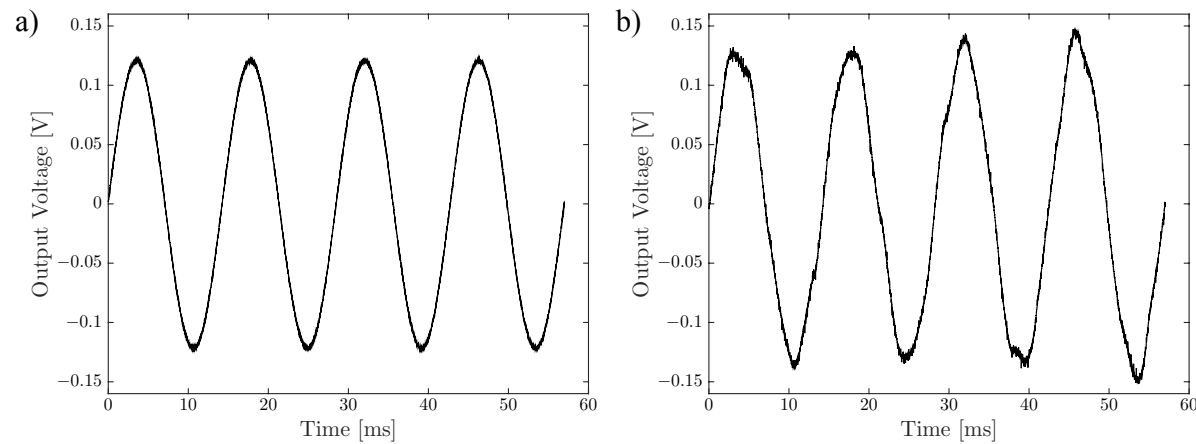

Figure 3.19: The output voltage signal from the sensor as a function of time for a) the pump rotational speed of 0 RPM, and b) the pump rotational speed of 900 RPM.

As described in Section 2.5, to analyze the change in the behaviour of the circuit as a function of the varying gap width inside the pump, the time domain signal $V_{\text {out }}(t)$ is then transformed into its frequency domain equivalent $\hat{V}_{\text {out }}(f)$ using the fast Fourier transform algorithm. The resulting FFT plot of the sensor's output signal at the pump rotational speed of 900 RPM is shown in Figure 3.20. The two peaks identified on the FFT plot correlate directly to the impeller's rotation speed. The first peak at $15 \mathrm{~Hz}$ is referring to the impellers rotational frequency and the 
second peak at $30 \mathrm{~Hz}$ refers to the blade passing frequency. Since there are two blades on the impeller of the pump, the blade's passing frequency is double the frequency of the impeller's rotational speed. To measure wear on the impeller blades, only the peak amplitudes are considered.

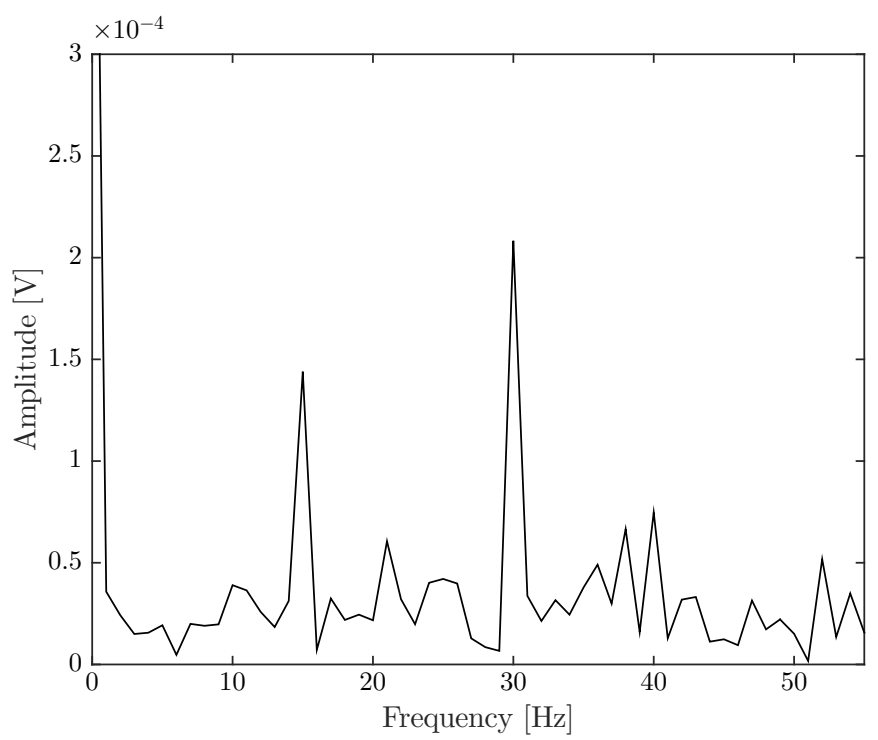

Figure 3.20: The FFT plot of the sensor output while the pump is operating at $900 \mathrm{RPM}$.

\subsubsection{Pump Rotational Speed Variation}

To ensure that the peaks identified on the FFT plot generated at the pump rotational speed of 900 RPM is consistent at various pump rotational speeds, measurements are taken while the rotational speed of the pump is varied from 450 RPM to 900 RPM. For these measurements the excitation frequency of the coil is set to $70 \mathrm{~Hz}$ while the signal is sampled at $50 \mathrm{kHz}$ sampling frequency for a duration of one second. The output signal is then transformed into the frequency domain as shown in Figure 3.21. The peaks visible on the plot are consistent with the rotational speed of the pump. 


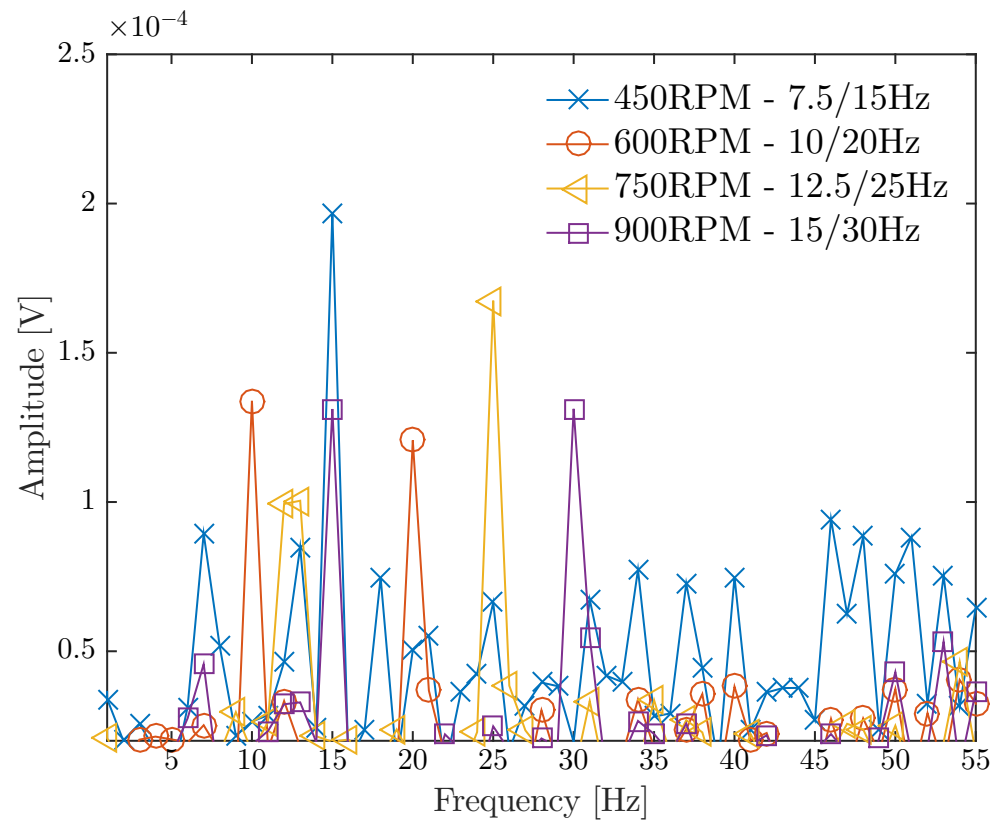

Figure 3.21: The amplitude spectral density of the sensor output for various pump rotational speeds.

\subsubsection{Signal to Noise Ratio (SNR)}

To analyze the quality of the output signal collected while the pump is in operation, the ratio of the signal with respect to the background noise or the signal to noise ratio

$$
\mathrm{SNR}=\frac{P_{\text {signal }}}{P_{\text {noise }}}
$$

is calculated as a function of the signal power $P_{\text {signal }}$ and the power of the noise $P_{\text {noise }}$. To calculate the SNR value of the sensor, only the signal collected at 900 RPM is taken into consideration as shown in Figure 3.20. To separate the noise from the raw signal, an interval-dependent haar wavelet denoising function is used in MATLAB. Figure 3.22 shows the plot of the denoised signal $\hat{V}_{\text {signal }}(f)$ and the noise $\hat{V}_{\text {noise }}(f)$. The amplitudes at the $15 \mathrm{~Hz}$ and $30 \mathrm{~Hz}$ frequencies are correlated to the absolute magnitude of the gap width and therefore the signal to noise ratio is calculated in the vicinity of these two frequencies and the total SNR 
is estimated by taking the average between the two resulting values. The SNR at $15 \mathrm{~Hz}$

$$
\mathrm{SNR}_{15}=\frac{\int_{f=13 \mathrm{~Hz}}^{17 \mathrm{~Hz}} S(f)_{\text {signal }} \cdot d f}{\int_{f=13 \mathrm{~Hz}}^{17 \mathrm{~Hz}} S(f)_{\text {noise }} \cdot d f}
$$

is measured by integrating the power spectral density of the denoised signal $S_{\text {signal }}(f)=$ $\left|\hat{V}_{\text {signal }}\right|^{2}$ divided by the power spectral density of the noise signal $S_{\text {noise }}(f)=$ $\left|\hat{V}_{\text {noise }}\right|^{2}$ between $13 \mathrm{~Hz}$ to $17 \mathrm{~Hz}$. The SNR value at the $15 \mathrm{~Hz}$ peak is calculated to be 14.30. Similarly, the SNR at $30 \mathrm{~Hz}$

$$
\mathrm{SNR}_{30}=\frac{\int_{f=28 \mathrm{~Hz}}^{32 \mathrm{~Hz}} S_{\text {signal }}(f) \cdot d f}{\int_{f=28 \mathrm{~Hz}}^{32 \mathrm{~Hz}} S_{\text {noise }}(f) \cdot d f}
$$

is also calculated to be 14.30 by integrating the signals from $28 \mathrm{~Hz}$ to $32 \mathrm{~Hz}$. This will produce an average SNR value of 14.30. The SNR can also be expressed in decibels

$$
\mathrm{SNR}_{\mathrm{dB}}=10 \cdot \log _{10}\left(\frac{P_{\text {signal }}}{P_{\text {noise }}}\right)
$$

and the calculated magnitude of the $\mathrm{SNR}_{\mathrm{dB}}$ is $11.6 \mathrm{~dB}$.

\subsubsection{Sampling Optimization}

To further improve the quality of the output signal and to increase the SNR of the sensor, the sampling frequency is varied and its effect on the noise level is observed as shown in Figure 3.23. By increasing the sampling frequency of the sensor while keeping the measurement duration constant at 1 second, the SNR increases leading 


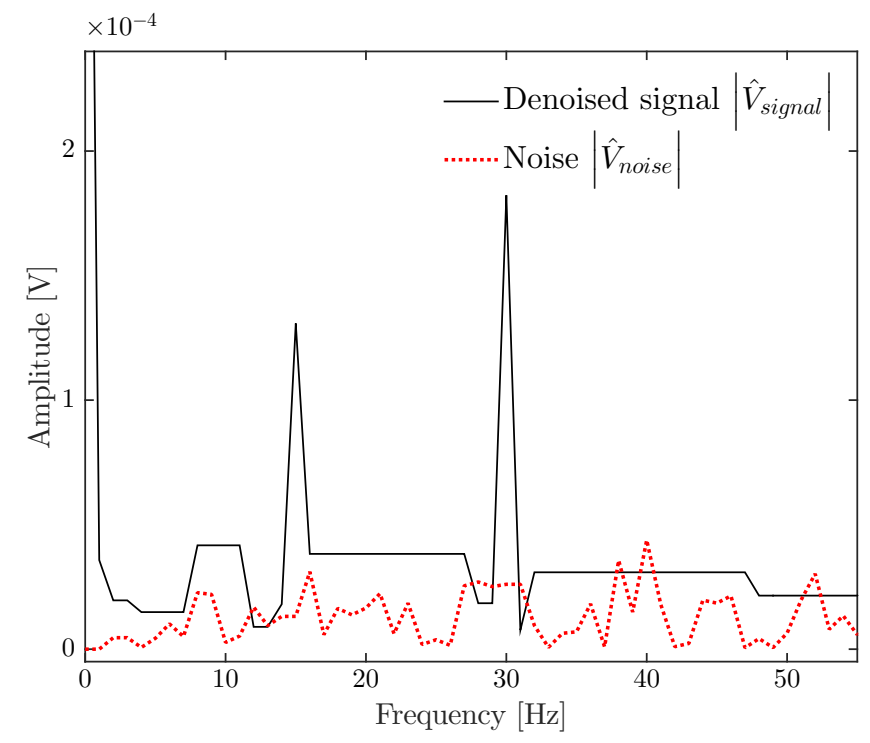

Figure 3.22: The plot of the output signal and the noise signal at the impeller rotational speed of $900 \mathrm{RPM}$. Signal is sampled at a sampling frequency of $50 \mathrm{~Hz}$ for a duration of 1 second.

to an improved output signal. This effect, however, declines as the sampling frequency is increased as shown in Figure 3.24. To ensure that the improvement in the sensor output is due to the sampling frequency and not the number of samples, the SNR is also calculated as a function of the sampling frequency for a constant number of samples at $1 \mathrm{M}$ data points as shown in Figure 3.25. In this study, the highest SNR is reached at $500 \mathrm{kHz}$ and the value of the $\mathrm{SNR}_{\mathrm{dB}}$ is $17.8 \mathrm{~dB}$ for one second of measurement. To observe the effect of measurement duration on the output of the sensor, the SNR is also plotted against the total number of samples used. As shown in Figure 3.26, the SNR is greatly improved with a higher sample number that is derived from a longer measurement duration.

\subsubsection{Data Collection}

To evaluate the functionality of the sensor and to validate the analytical and simulation results obtained earlier in this thesis, the change in the sensor's output signal 

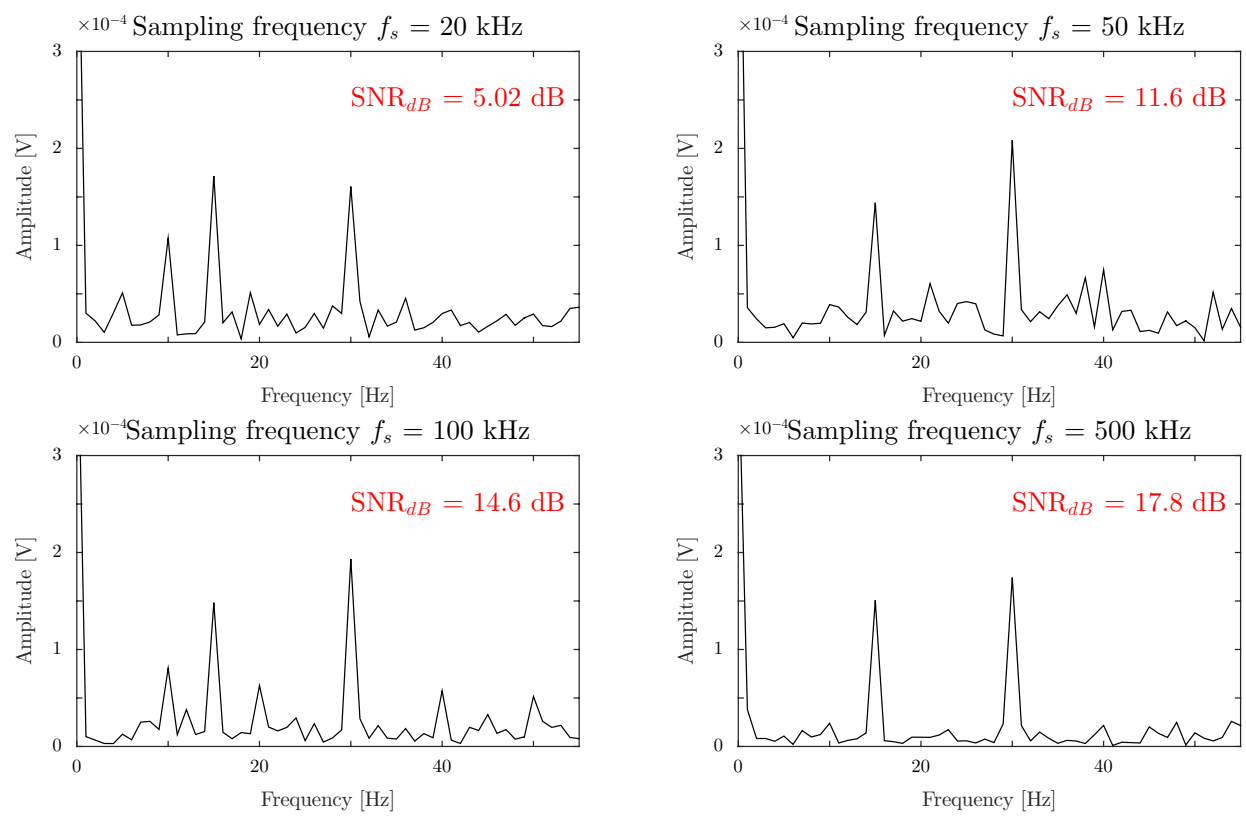

Figure 3.23: Sensor output collected at different sampling frequencies of $20 \mathrm{kHz}, 50 \mathrm{kHz}, 100 \mathrm{kHz}$, and $500 \mathrm{kHz}$. The SNR value of each data is labeled on the plot. All measurements were taken during 1 second with a pump rotational speed of 900 RPM.

is examined as a function of the varying gap width inside the pump. As explained in Section 3.3 the sensor is calibrated by incrementally increasing the varying gap inside the pump at $0.75 \mathrm{~mm} \pm 0.05 \mathrm{~mm}$ increments from $0.40 \mathrm{~mm} \pm 0.05 \mathrm{~mm}$ to $2.65 \mathrm{~mm} \pm 0.20 \mathrm{~mm}$ and measurements are taken at each gap width. To correlate the output of the sensor with the width of the varying gap, the amplitude of the FFT signal at $30 \mathrm{~Hz}$ is plotted against the amplitude at $15 \mathrm{~Hz}$ for each 1 second of measurement. The resulting scatter plot is shown in Figure 3.27 and the mean and standard deviations of the scatter plot are shown in Figure 3.28. The amplitude at the $15 \mathrm{~Hz}$ frequency is clearly correlated to the varying gap width inside the pump, whereas the amplitude at the $30 \mathrm{~Hz}$ frequency has no evident relation with the gap. This behaviour in the sensor indicates that the two blades on the impeller are different in thickness and this could have been caused by the accumulation of uneven wear on the blades. Figure 3.29 shows the amplitude of the output signal at $15 \mathrm{~Hz}$ 


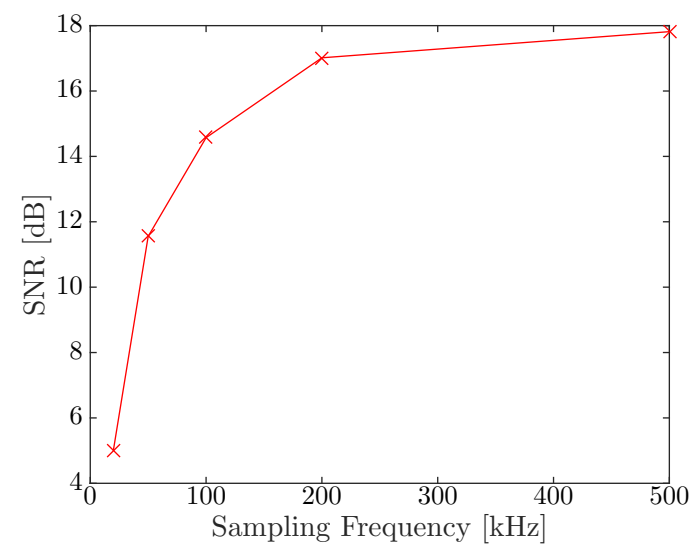

Figure 3.24: Signal to noise ratio in $[\mathrm{dB}]$ as a function of the sampling frequency for a one-second measurement on the pump running at 900 RPM.

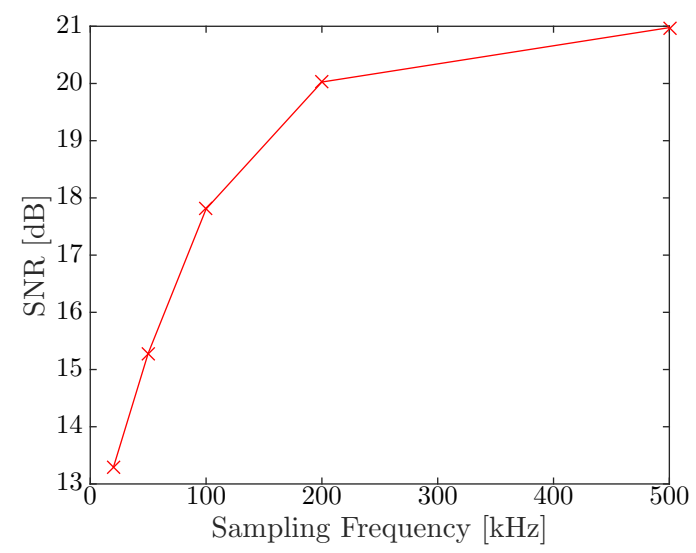

Figure 3.25: Signal to noise ratio in $[\mathrm{dB}]$ as a function of the sampling frequency for $1 \mathrm{M}$ data points.

versus the magnitude of the varying gap, with the error bars and the uncertainty in the gap width identified at each data point. The average size of the error bar is also compared against the duration of the measurement and it is observed that as the sampling time increases, the size of the error bar decreases leading to a higher accuracy and resolution of the output signal. 


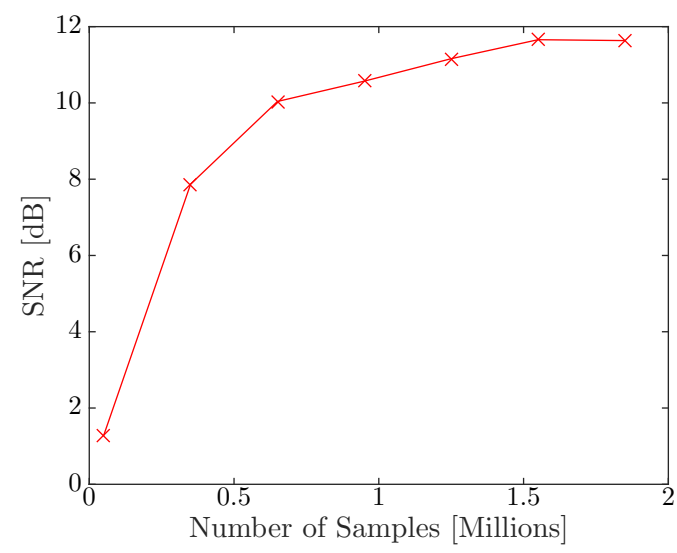

Figure 3.26: Signal to noise ratio in $[\mathrm{dB}]$ as a function of the total number of samples with a fixed sampling frequency of $50 \mathrm{kHz}$.

It is noteworthy to mention that many other alternative methods for measuring the peak amplitude are also considered such as the ratio between the peak amplitude at both frequencies with respect to the amplitude at $70 \mathrm{~Hz}$ (the excitation frequency), the ratio between the peak amplitude to the RMS value of the noise, and also the difference between the amplitude of the signal and the noise level. The resulting plots, however, did not improve in neither of these scenarios. The plots are presented in Appendix C.

To compare the experimental data with the simulation results generated as described in Chapter 2, the calibration curve of the sensor (Figure 3.29) is plotted against the simulation results as shown in Figure 3.30 In this comparison, the simulation results are multiplied by a gain of 12 to account for the amplification created using the external capacitor in the sensor's circuit. As expected, the simulation results yield a considerably higher sensitivity primarily due to the inevitable gaps created inside the flux guide assembly during the fabrication process and in the contacts between the sensor and the pump that were not included in the simulation model. 


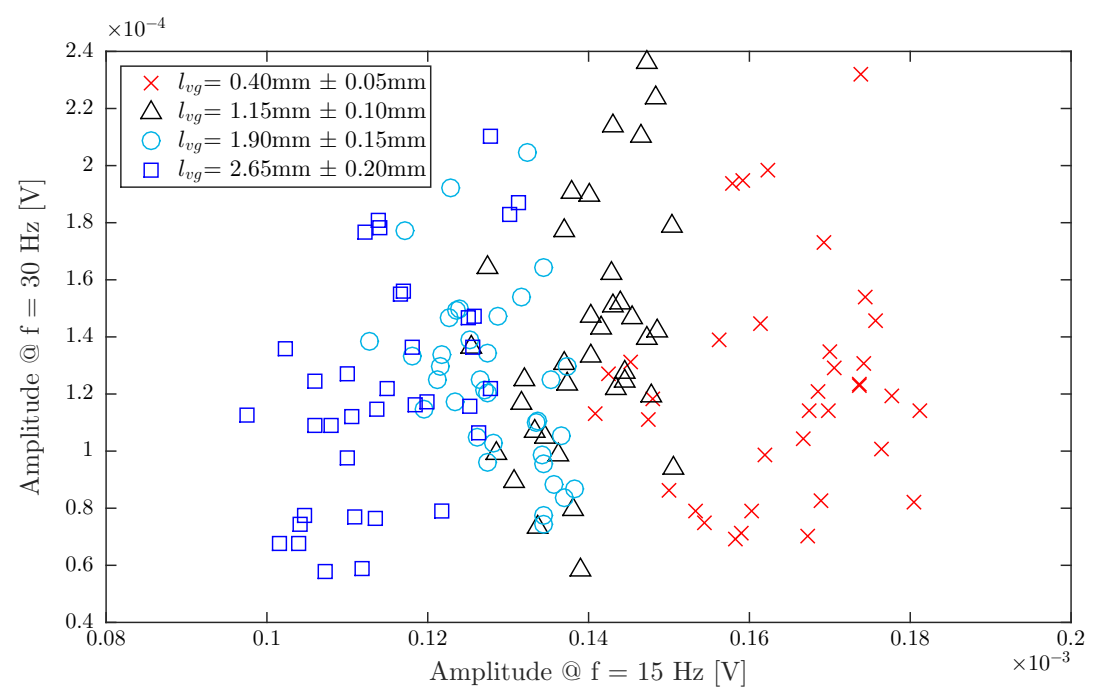

Figure 3.27: Scatter plot of the sensor output signal at $30 \mathrm{~Hz}$ versus the output signal $15 \mathrm{~Hz}$ for various gap widths. Each data point refers to 1 second of measurement collected at $500 \mathrm{kHz}$ sampling frequency. There are 36 data points for each gap width.

\subsubsection{Wear Detection}

Once the sensor is calibrated, the magnetic wear sensor can be used to estimate the magnitude of wear inside the centrifugal pump while the pump is in operation. To do this, data will be collected every few days for a duration of no more than one minute and the resulting data is used to estimate the change in the width of the impeller blade. To measure the magnitude of wear based on the sensor's output signal, the collected data is transformed into the frequency domain and the amplitude of the resulting signals at $15 \mathrm{~Hz}$ is compared against the acquired calibration curve shown in Figure 3.29. Based on the calibration curve, the sensor has an average sensitivity of $\bar{S}=\frac{\Delta V}{\Delta l}=0.022 \mathrm{mV} / \mathrm{mm}$. With an average error bar size of $\delta V=$ $0.0082 \mathrm{mV}$, the resolution achieved by the sensor is $\delta l=\frac{\delta V}{\bar{S}}=0.38 \mathrm{~mm}$. Since wear measurements are taken with reference to the initial clearance between the impeller and the side plate, the accuracy of the sensor is limited by the accuracy of the initial gap measurement and the resolution of the sensor. 


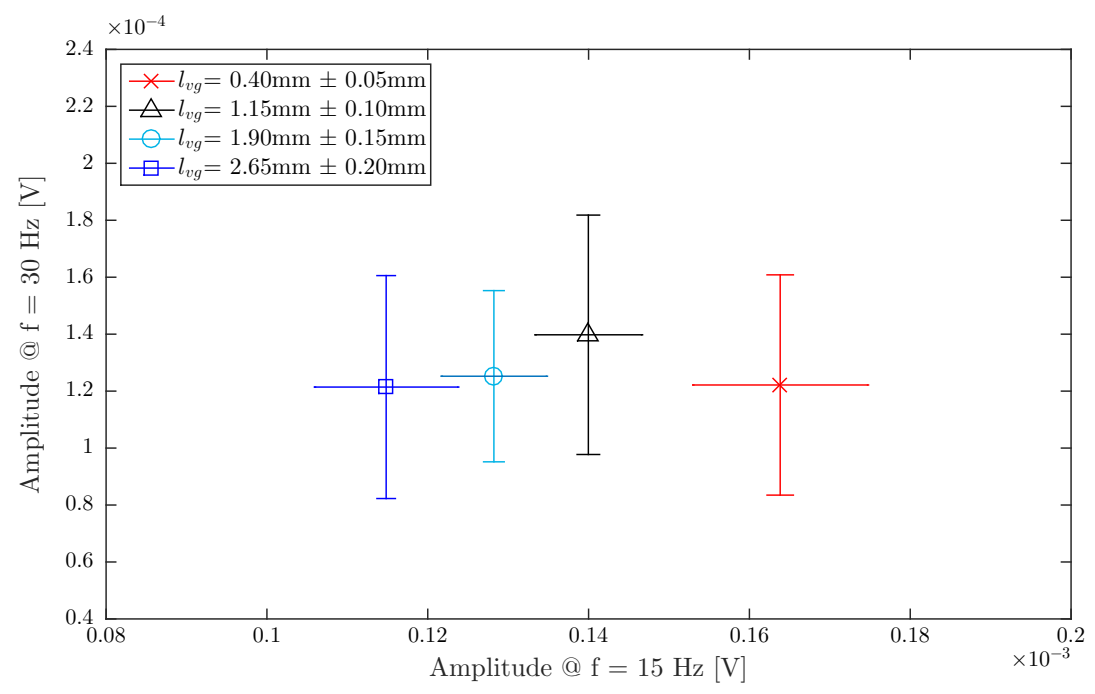

Figure 3.28: Mean and the standard deviation of the scatter plot for the sensor output signal at $30 \mathrm{~Hz}$ versus the output signal $15 \mathrm{~Hz}$ at various gap widths. The sampling frequency is set to $500 \mathrm{kHz}$ and measurements are taken for a duration of 36 seconds.

The reproducibility and the repeatability of the data collected from the sensor are also very crucial when assessing the performance of the wear sensor. To test the reproducibility of the sensor output, the assembly is completely removed from the pump and placed back again three times while measurements are taken each time the sensor is affixed to the pump. The samples are collected at $500 \mathrm{kHz}$ sampling frequency for a duration of one second while the pump is running at $900 \mathrm{RPM}$. To quantify the reproducibility of the sensor output, the voltage amplitude at $15 \mathrm{~Hz}$ frequency in the FFT plot for each data set is obtained and the standard deviation between the resulting values is calculated. In this case, the standard deviation is calculated to be $6.60 \mu \mathrm{V}$. Similarly, to quantify the repeatability of the sensor output, 10 sets of one-second measurements are taken from the pump with the same sampling conditions while the sensor is kept affixed to the pump. Using the same procedure as in the case of the reproducibility check, the standard deviation between the resulting values is calculated to be $10.86 \mu \mathrm{V}$. As it can be observed, both magnitudes are in the same order of magnitude but more experiments are required 


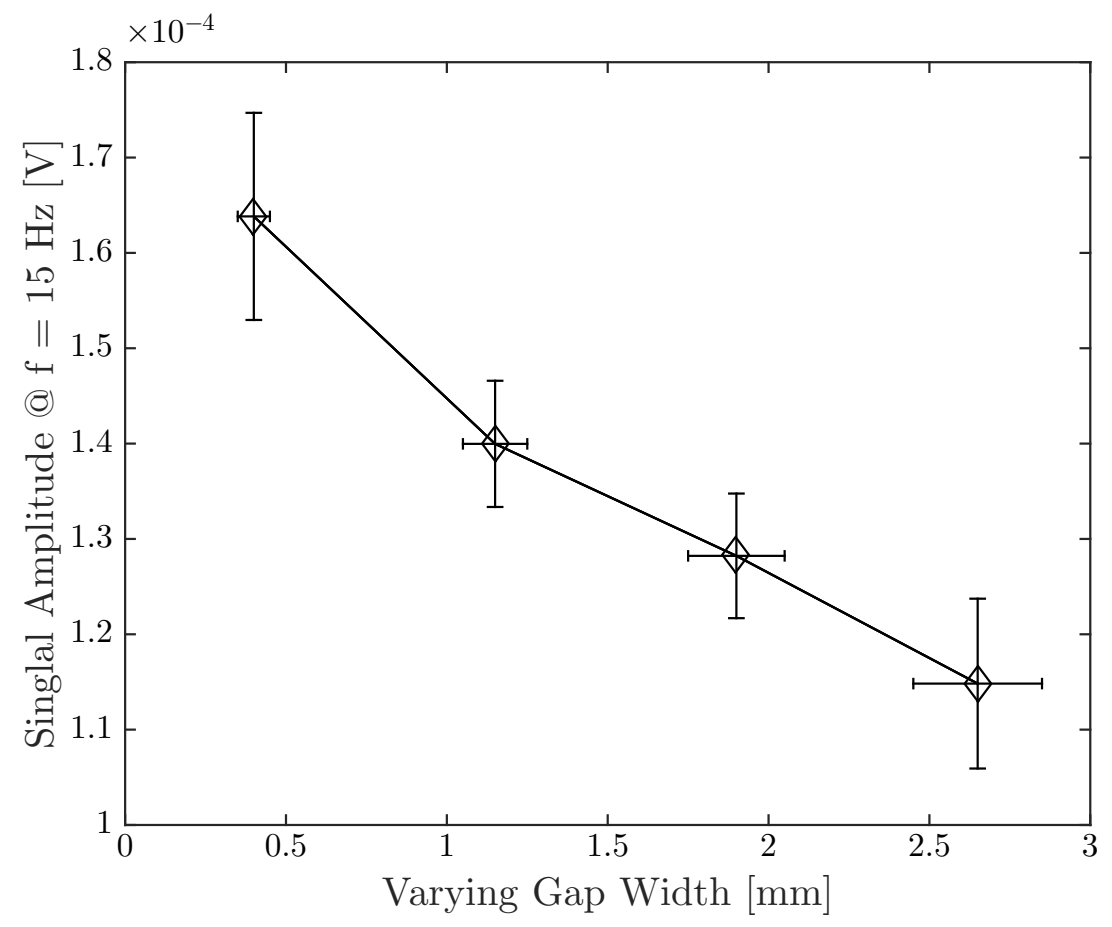

Figure 3.29: The mean amplitude of the sensor's output signal at $15 \mathrm{~Hz}$ versus the magnitude of the varying gap width. The error bar and the uncertainty of the gap width for each data point is shown on the plot.

to derive a more accurate estimation of the error. In comparison to the resolution and the sensitivity of the sensor output, both the reproducibility and the repeatability of the sensor are well within the acceptable range. 


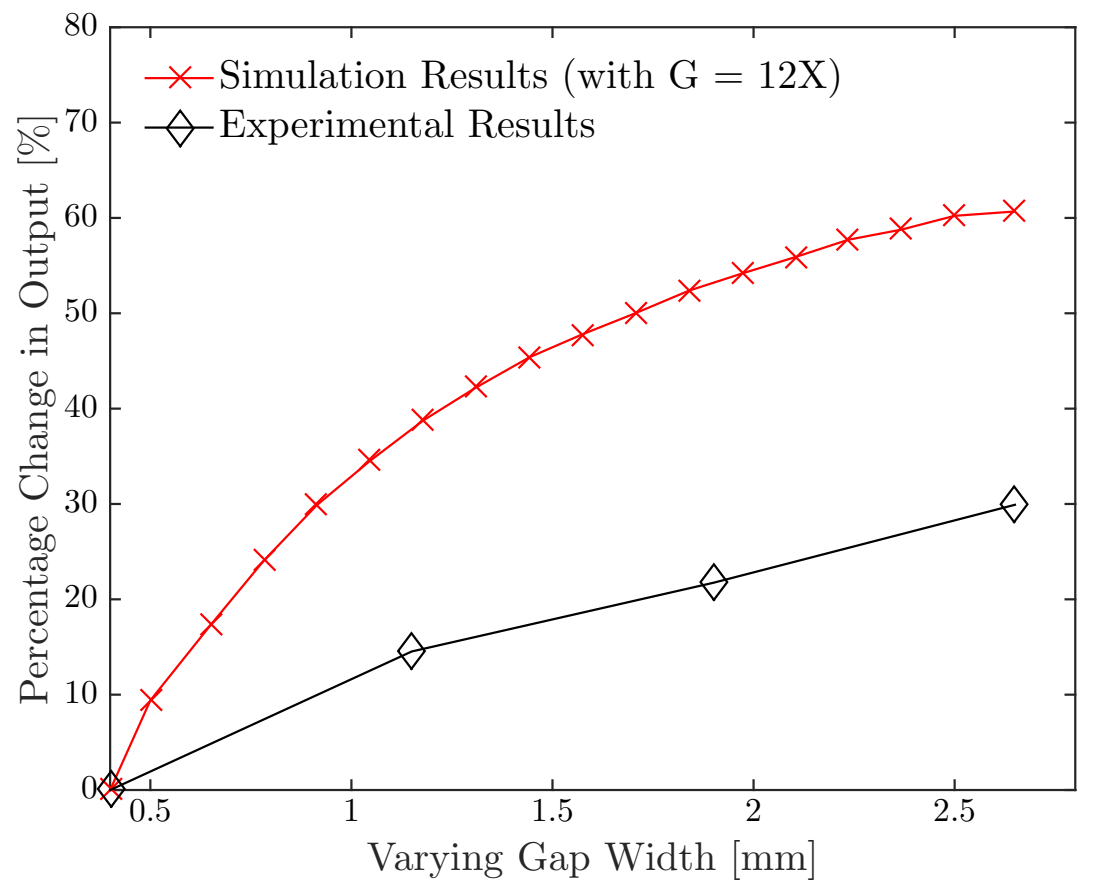

Figure 3.30: Comparison between the experimental results and the simulation results. The plot shows the percentage change in the output of the sensor as a function of the varying gap width as it increases from $0.4 \mathrm{~mm}$ to $2.65 \mathrm{~mm}$. 


\section{Chapter 4}

\section{Conclusions and Future Work}

\subsection{Conclusions}

In this study, a live magnetic wear sensor is designed and tested that allows for wear measurement inside centrifugal pumps. The sensor is in a form of a clamping mechanism attached to a portable instrumentation box allowing it to be installed on any operational centrifugal pump. The sensor designed has a signal to noise ratio of $17.8 \mathrm{~dB}$ with an average sensitivity of $0.022 \mathrm{mV} / \mathrm{mm}$ and a resolution of $0.38 \mathrm{~mm}$. The sensitivity and the resolution achieved by this sensor make it suitable for wear measurements of industrial pumps inside a plant. The standard deviation values referring to the reproducibility and the repeatability of the sensor are $6.60 \mu \mathrm{V}$ and $10.86 \mu \mathrm{V}$ respectively.

The main limitation of this sensor is that it requires recalibration for every pump it is placed on which could be a time consuming process. Also, the sensor is only functional for open-impeller centrifugal pumps. This class of pumps are most commonly used inside process plants for transporting slurry fluids or fluids with solid inconsistencies.

This sensor, however, introduces many other opportunities for similar wear or gap measurements. Wear measurement inside rotating machinery, for instance, where there are two parallel plates such as a pulp refiner could be achieved by this 
sensor. Clearance or gap measurement inside machinery is also achievable by this sensor in cases where access to the inside of the component is limited.

\subsection{Future Work}

The sensor developed in this project is proven to be functional while testing its performance on a single centrifugal pump in a pilot plant. To further improve the performance of the sensor and to make it into a final product, there are several improvements that can be done.

\section{Flux Guide Material}

Because of the time and cost limitations of this project, there was only one prototype of the sensor fabricated and this limited the potentials achievable with the sensor concept. The material used in the flux guide assembly was selected based on extreme design parameters to ensure the functionality of the sensor. Since the cost of the flux guide material is the main part of the total cost of the sensor as shown in Table 3.1, to lower the cost of fabrication, cheaper materials can be used without sacrificing a large fraction of the sensitivity. As it could be seen from the analytical and simulation results, a material with a relative permeability of $\mu_{r}=5,000$ is sufficient for the operation of the sensor. Also, since the sensor needs to be installed inside a plant for a duration of several years, the body of the sensor needs to be rigid and durable. The material used for the flux guide assembly in this project is highly brittle and can easily break once placed on a pump. The design of the flux guide assembly can also be further improved to minimize the air gaps inside the body. Currently the two moving cylindrical rods inside the sensor are not tightly connected to the rest of the assembly and this creates an air gap inside the flux guide. 


\section{Sensor Probes}

One of the most important components of the flux guide assembly that was not studies thoroughly in this project is the tip of the sensor probes that connect the sensor to the pump. To make the wear measurements repeatable with a higher sensitivity, a firm and a more rigid sensor probe needs to be designed and implemented to first, minimize the contact gap between the probe and the pump, and second, to stay in contact with the pump with the presence of vibrations and external disturbances from the surroundings. As a recommendation to overcome this problem, the designed magnetic polymer as described in Section 3.3 can be used to create an adapter on the pump for the sensor probes. The polymer can form the shape of the pump on one end to minimize any air gaps between the pump and the sensor, and on the other end, it can embody a mouth piece the same size as the sensor probes. The adapter can be bonded to the pump housing by using the magnetic epoxy as described in Section 3.1

\section{Clamping Mechanism}

Since the body of the sensor is relatively heavy, a proper clamping mechanism needs to be designed to hold the sensor on the pump. For this study, the sensor was affixed to the pump by a hanging mechanism that supported the sensor from an above fixture. Although this method worked very well for this project, if the sensor is to be installed on every pump inside a plant, this method needs to be improved. As an example of a potential clamping mechanism, an additional C-clamp can be used over the sensor to hold the sensor on the pump. Since the size of the sensor is rather large, the additional C-clamp might need to be fabricated specifically for this purpose.

\section{Data Collection}

It is believed that the sampling frequency plays an important role in reducing the noise level in the output signal. As Figure 3.23 shows, the SNR is significantly improved when the sampling frequency is increased from $20 \mathrm{kHz}$ to $500 \mathrm{kHz}$. The 
sampling frequency in this project was limited to $500 \mathrm{kHz}$ due to hardware limitations. To potentially improve the signal quality, higher sampling rates must be tested and their effect on the performance of the sensor needs to be verified.

\section{Sensor Calibration}

Because of time constraints in this project, the gap inside the pump is manually increased to simulate a wear effect inside the pump. To further validate the functionality of the sensor, the assembly needs to be installed on a pump for a longer duration to observe the effect of erosion on the output of the sensor. As an alternative approach, the impeller can also be replaced with used impellers each having a different depth of wear on the blades that are physically measured prior to being placed inside the pump.

\section{Pump Efficiency}

To monitor the effect of wear on the efficiency of centrifugal pumps and to quantify the energy losses associated with wear, pump efficiency needs to be monitored along with the impeller wear while the pump is in operation.

\section{Field Test}

To make this sensor operable inside a process plant with little to no supervision, there are several additional steps that needs to be taken both on the hardware side and the software. In regards to the physical assembly of the sensor, a more rigid and stable clamping mechanism is to be designed to ensure the sensor stays in contact with the pump given the potential disturbances inside an operating plant. As for the software, a simple program needs to be written that enables the sensor to automatically collect data every few days and interpret the data so that the operator can assess the integrity of the pump without any additional work. 


\section{Bibliography}

[1] R. Khoie, B. Gopaluni, J. A. Olson, and B. Stoeber, "A magnetic sensor to measure wear in centrifugal pumps," in SENSORS, 2015 IEEE, Nov 2015, pp. $1-4$. $\rightarrow$ pages iv

[2] T. Sahoo and A. Guharoy, "Energy cost savings with centrifugal pumps," World Pumps, vol. 2009, no. 510, pp. 35-37, 2009. $\rightarrow$ pages 1

[3] M. Pemberton, "Strategies for optimizing pump efficiency and lcc performance," 2003. $\rightarrow$ pages 1

[4] L. Frenning, Pump life cycle costs: A guide to LCC analysis for pumping systems. Hydraulic Institite \& Europum, 2001. $\rightarrow$ pages 1

[5] M. Holloway, C. Nwaoha, and O. Onyewuenyi, Process Plant Equipment: Operation, Control, and Reliability, ser. EngineeringPro collection. Wiley, 2012. $\rightarrow$ pages 1

[6] C. Lei, Z. Yiyang, W. Zhengwei, X. Yexiang, and L. Ruixiang, "Effect of axial clearance on the efficiency of a shrouded centrifugal pump," Journal of Fluids Engineering, vol. 137, no. 7, p. 071101, 2015. $\rightarrow$ pages 1

[7] G. Wood, H. Welna, and R. Lamers, "Tip-clearance effects in centrifugal pumps,” Journal of Fluids Engineering, vol. 87, no. 4, pp. 932-939, 1965. $\rightarrow$ pages 2

[8] Y. Khalid and S. Sapuan, "Wear analysis of centrifugal slurry pump impellers," Industrial Lubrication and Tribology, vol. 59, no. 1, pp. 18-28, 2007. $\rightarrow$ pages 2

[9] F. Schmaljohann, D. Hagedorn, A. Bu, R. Kumme, and F. Löffler, "Thin-film sensors with small structure size on flat and curved surfaces," Measurement Science and Technology, vol. 23, no. 7, p. 074019, 2012. $\rightarrow$ pages 4 
[10] G. Rutelli and D. Cuppini, "Development of wear sensor for tool management system," Journal of Engineering Materials and Technology, vol. 110, pp. 59-62, 1988/01/01. $\rightarrow$ pages 4

[11] N. Ghosh, Y. Ravi, A. Patra, S. Mukhopadhyay, S. Paul, A. Mohanty, and A. Chattopadhyay, "Estimation of tool wear during cnc milling using neural network-based sensor fusion," Mechanical Systems and Signal Processing, vol. 21, no. 1, pp. 466-479, 2007. $\rightarrow$ pages 4

[12] H. Guerrero, "Magnetic proximity sensor for measuring gap between opposed refiner plates," Patent, Aug. 21, 1990, US Patent 4,950,986. $\rightarrow$ pages 5

[13] D. Dodson-Edgars, "Gap, wear and tram measurement system and method for grinding machines," Apr. 11 1989, US Patent 4,820,980. $\rightarrow$ pages 5

[14] N. Mitsumune, I. Saito, S. Mochizuki, Y. Abe, T. Isoyama, H. Nakagawa, T. Ono, A. Kouno, A. Sugino, and T. Chinzei, "Fundamental study to develop a fiber-optic gap sensor for a rotary undulation pump," Journal of Artificial Organs, vol. 10, no. 4, pp. 231-235, 2007. $\rightarrow$ pages 5

[15] S. Liang and D. Dornfeld, "Tool wear detection using time series analysis of acoustic emission," Journal of Engineering for Industry, vol. 111, no. 3, pp. 199-205, 1989. $\rightarrow$ pages 5

[16] L. Alfayez, D. Mba, and G. Dyson, "The application of acoustic emission for detecting incipient cavitation and the best efficiency point of a $60 \mathrm{kw}$ centrifugal pump: case study," Ndt \& E International, vol. 38, no. 5, pp. 354-358, 2005. $\rightarrow$ pages 6

[17] P. Campbell, Permanent Magnet Materials and Their Application. Cambridge University Press, 1996. $\rightarrow$ pages 9, 15

[18] L. Matsch, Capacitors, Magnetic Circuits, and Transformers, ser. Prentice-Hall electrical engineering series. Prentice-Hall, 1964. $\rightarrow$ pages 25

[19] C. R. Nave. (2011) Relative permeability - hyperphysics. (retrieved on 09/05/2014). [Online]. Available:

http://hyperphysics.phy-astr.gsu.edu/hbase/solids/ferro.html $\rightarrow$ pages 32

[20] "Astm b258 - 14, specification for nominal diameters and cross-sectional areas of AWG sizes of solid round wires used as electrical conductors." $\rightarrow$ pages 41 
[21] A. Massarini and M. K. Kazimierczuk, "Self-capacitance of inductors," IEEE Transactions on Power Electronics, vol. 12, no. 4, pp. 671-676, Jul 1997. $\rightarrow$ pages 42

[22] "Gluing of ferrite cores," 2002, (retrieved on 03/22/2015). [Online]. Available: http://www.elnamagnetics.com/wp-content/uploads/library/ Elna-Magnetics/Gluing_of_Ferrite_Cores.pdf $\rightarrow$ pages 55 


\section{Appendix A}

\section{Computer Simulations}

\section{A.1 Simplified Model}

For the numerical analysis of the sensor model, Comsol Multiphysics is used to model the pump and the sensor. For rapid modeling and the proof of concept, initially a simplified model of the pump is used to derive the change in the magnitude of the flux density as a function of the varying gap width inside the pump. Figure A.1 represents the Comsol model used to plot the change in the magnetic flux density as a function of the gap width for the electromagnet sensor model. Similarly, Figure A.2 is used to derive the change in the inductance of the magnetic coil as a function of the varying gap in the case of the inductance model. It can be noticed that the inductance model does not contain a secondary air gap inside the flux guide assembly since there is no need for a Hall-effect sensor to be in place. 


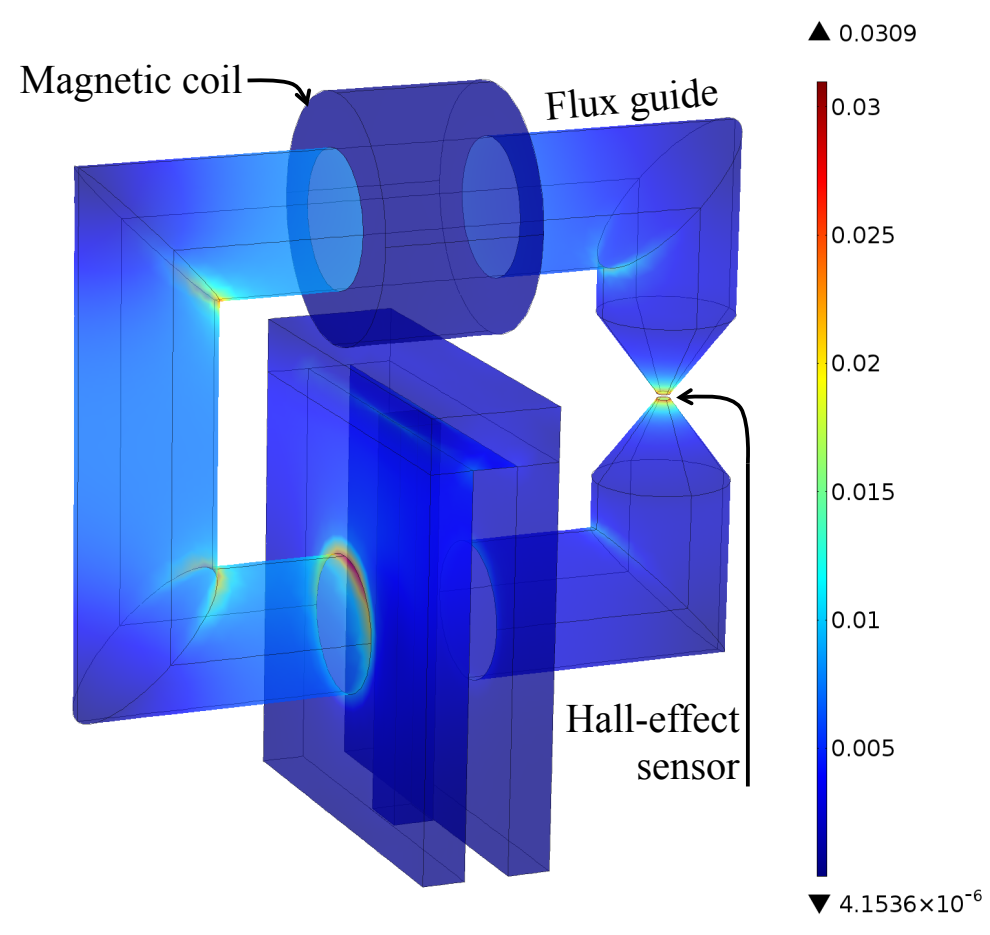

Figure A.1: Magnitude of flux density [T] inside the sensor and the simplified pump for the analysis of the electromagnet sensor model generated using Comsol Multiphysics. 


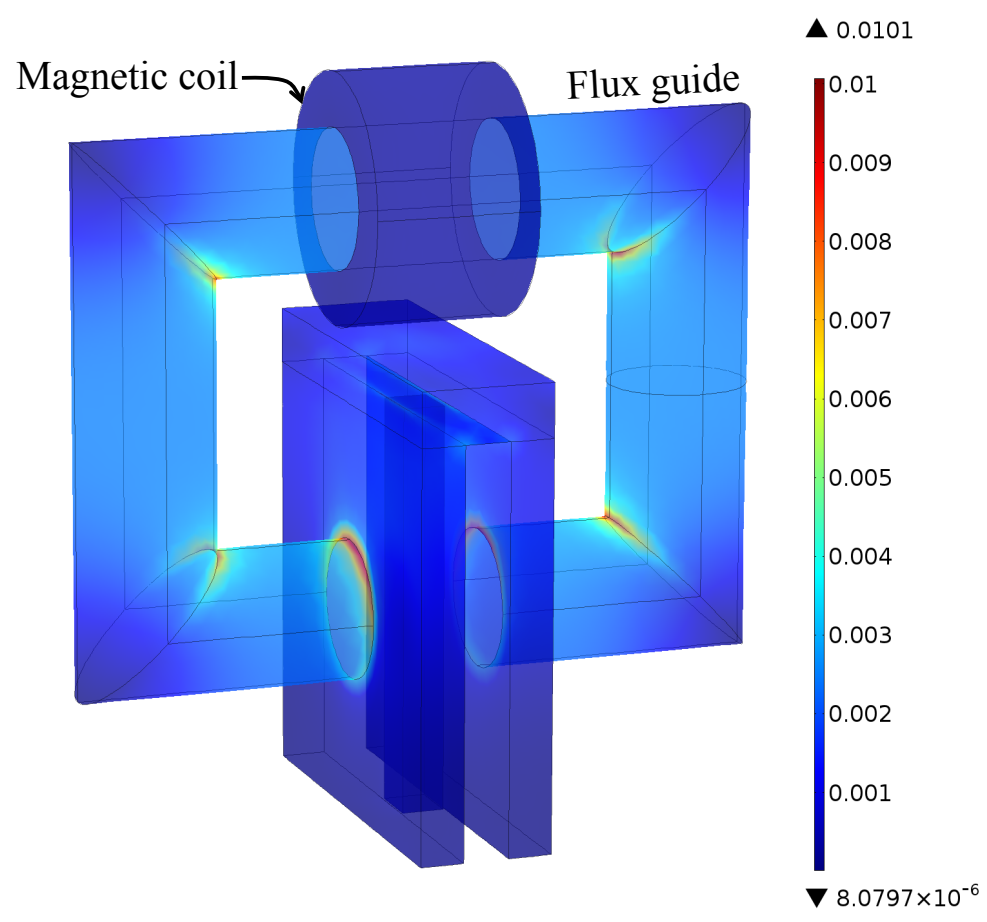

Figure A.2: Magnitude of flux density [T] inside the sensor and the simplified pump for the analysis of the inductance sensor model generated using Comsol Multiphysics.

\section{A.2 Advanced Model}

To monitor the magnitude of flux inside the clamping mechanism designed in Chapter 2, the sensor body and the pump are modeled in Comsol Multiphysics. For the finite element simulations, the magnetic coil is excited at $100 \mathrm{~Hz}$ using a $1 \mathrm{~V}$ voltage signal. Figure A.3 represents the magnitude of flux inside the sensor and the pump. As it can be noticed from the simulation model, there is no saturation occurring inside the flux guide geometry as the saturation flux density for the M100 material is $0.42 \mathrm{~T}$. 
$\Delta 8.5603 \times 10^{-3}$
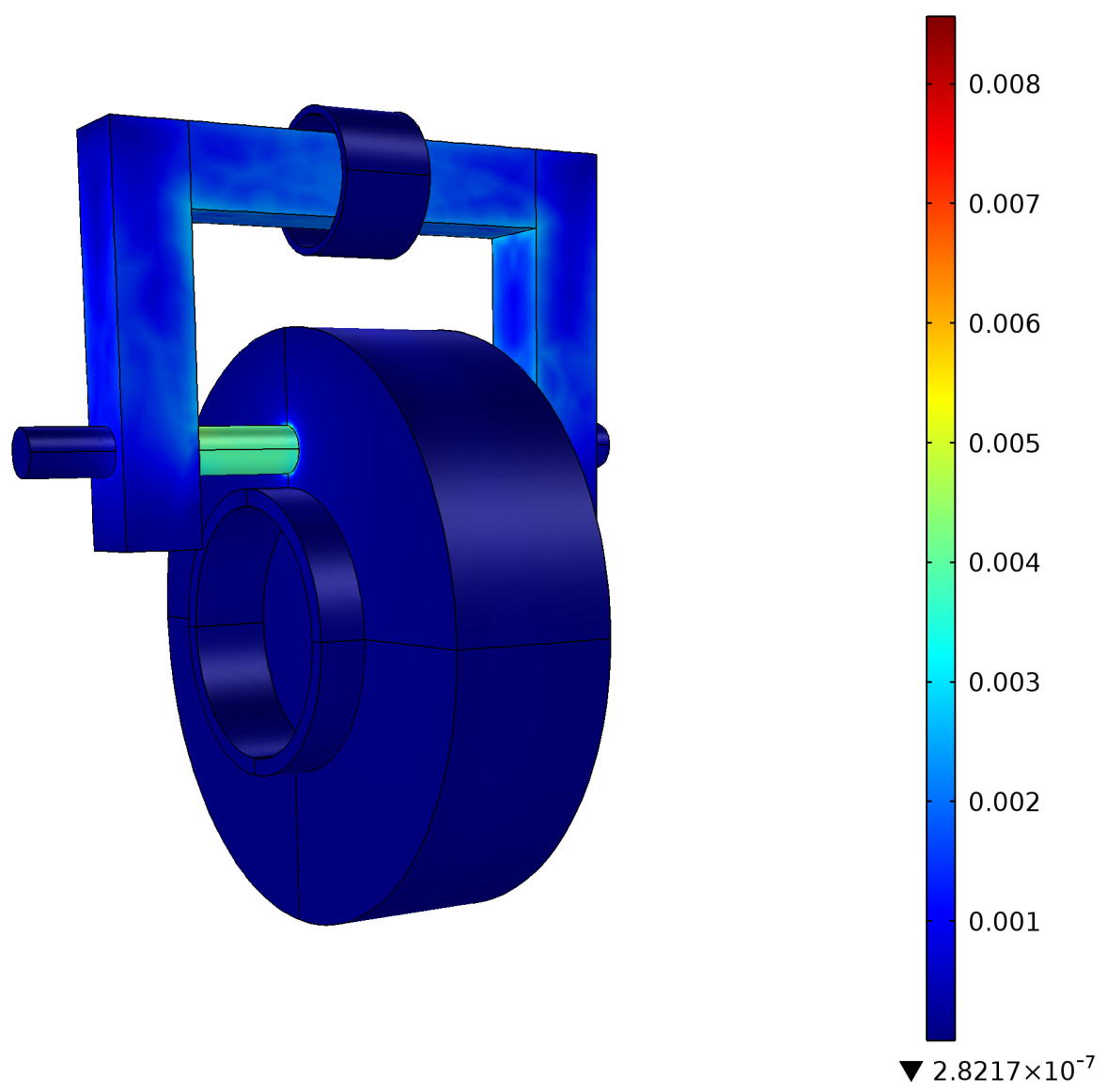

Figure A.3: Magnitude of the flux density [T] inside the flux guide assembly. The saturation flux density for M100 material is $0.42 \mathrm{~T}$ 


\section{Appendix B}

\section{MATLAB Code}

For this project, MATLAB software is used to derive all the analytical results presented in the thesis document. For reference, only the MATLAB codes that are more complex have been included in the Appendix section. This chapter includes the codes related to FFT calculations, signal denoising, scatter plots, error bar estimation, and calibration curves.

\section{B.1 FFT Calculation}

The MATLAB code generated to calculate the FFT plots represented in Section 3.4 is represented below.

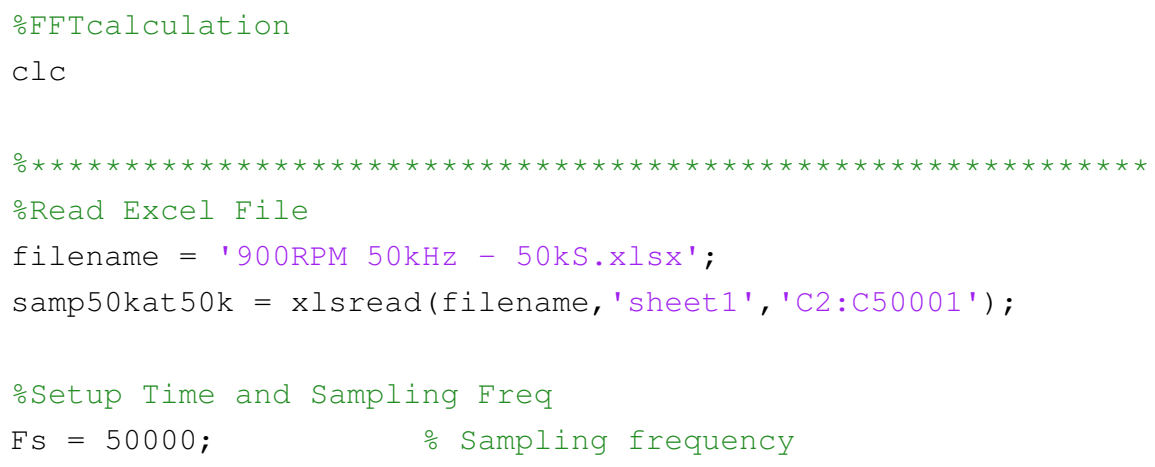




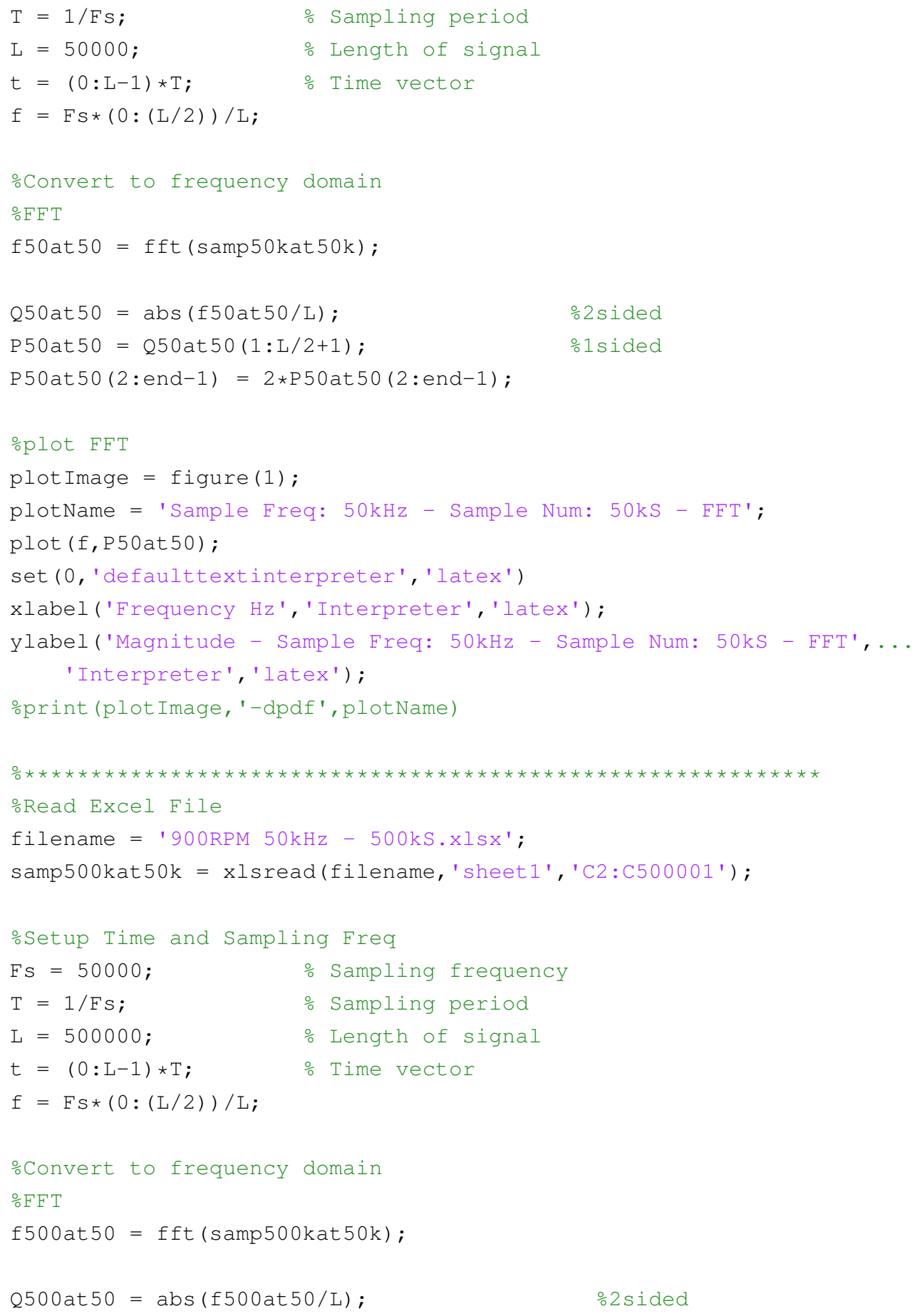




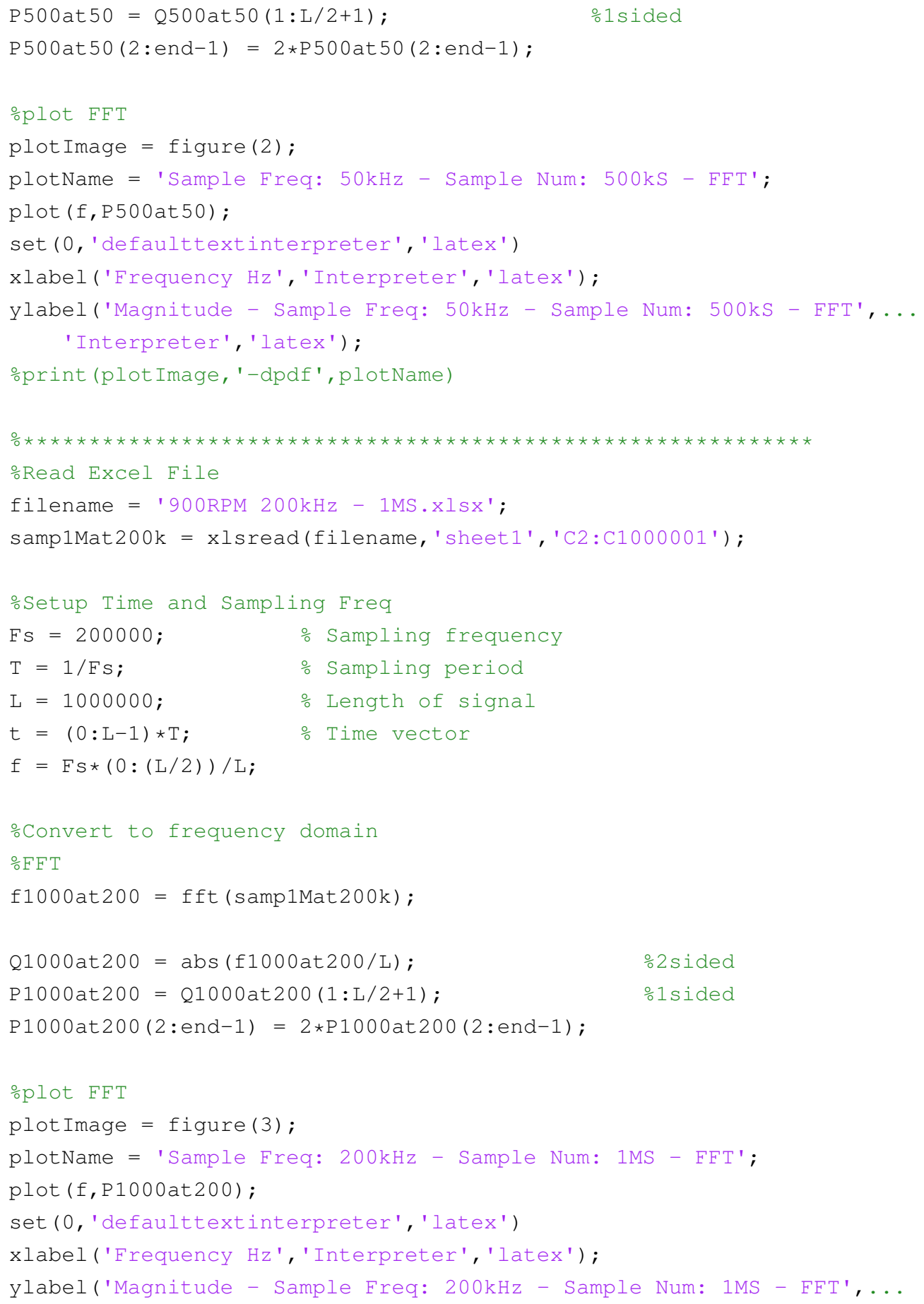




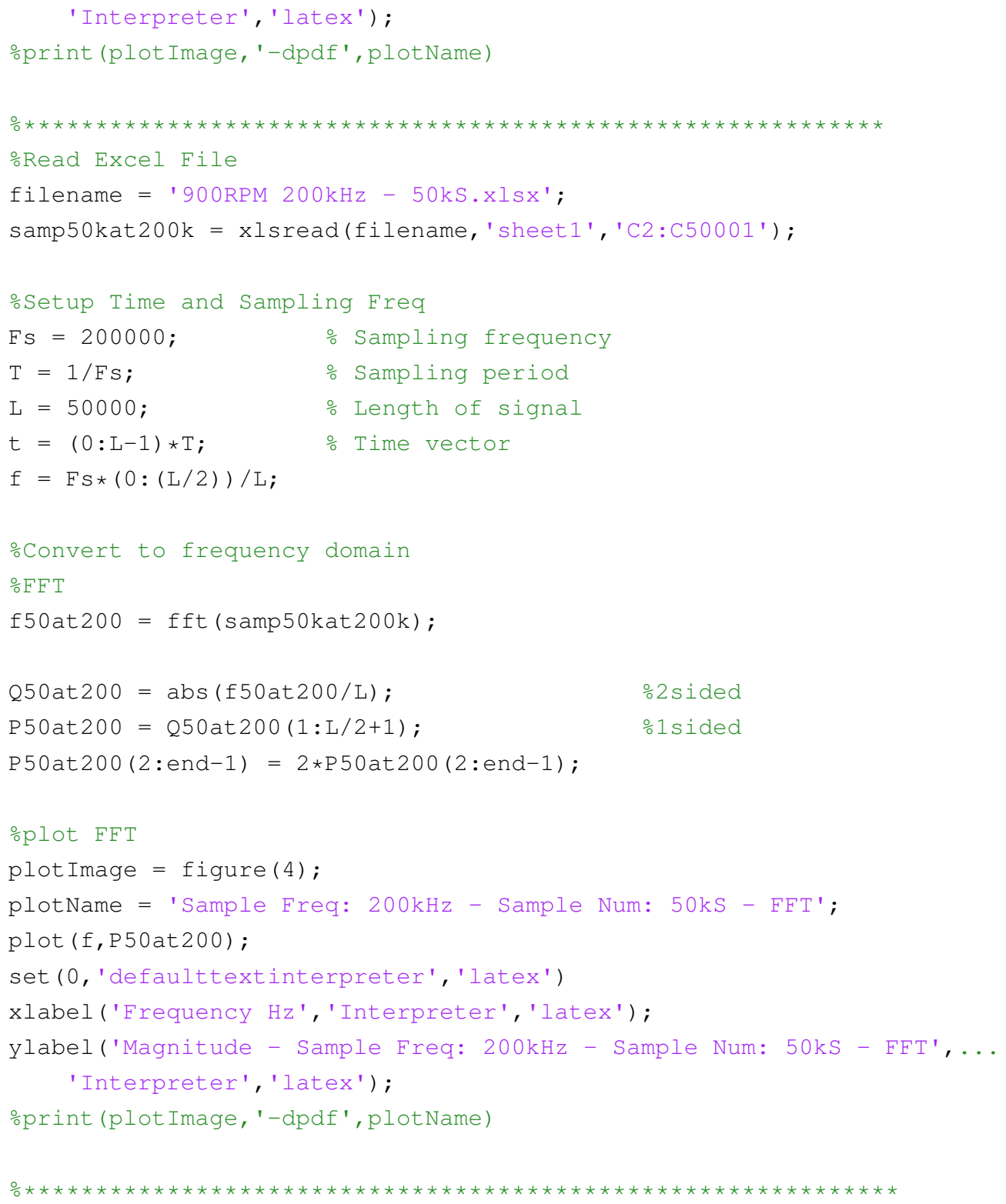




\section{B.2 Signal Denoising and SNR Calculation}

Once the FFT plot is calculated using the original time series data, the signal is denoised and the SNR value is estimated using the MATLAB software. The MATLAB code used for this purpose is represented below.

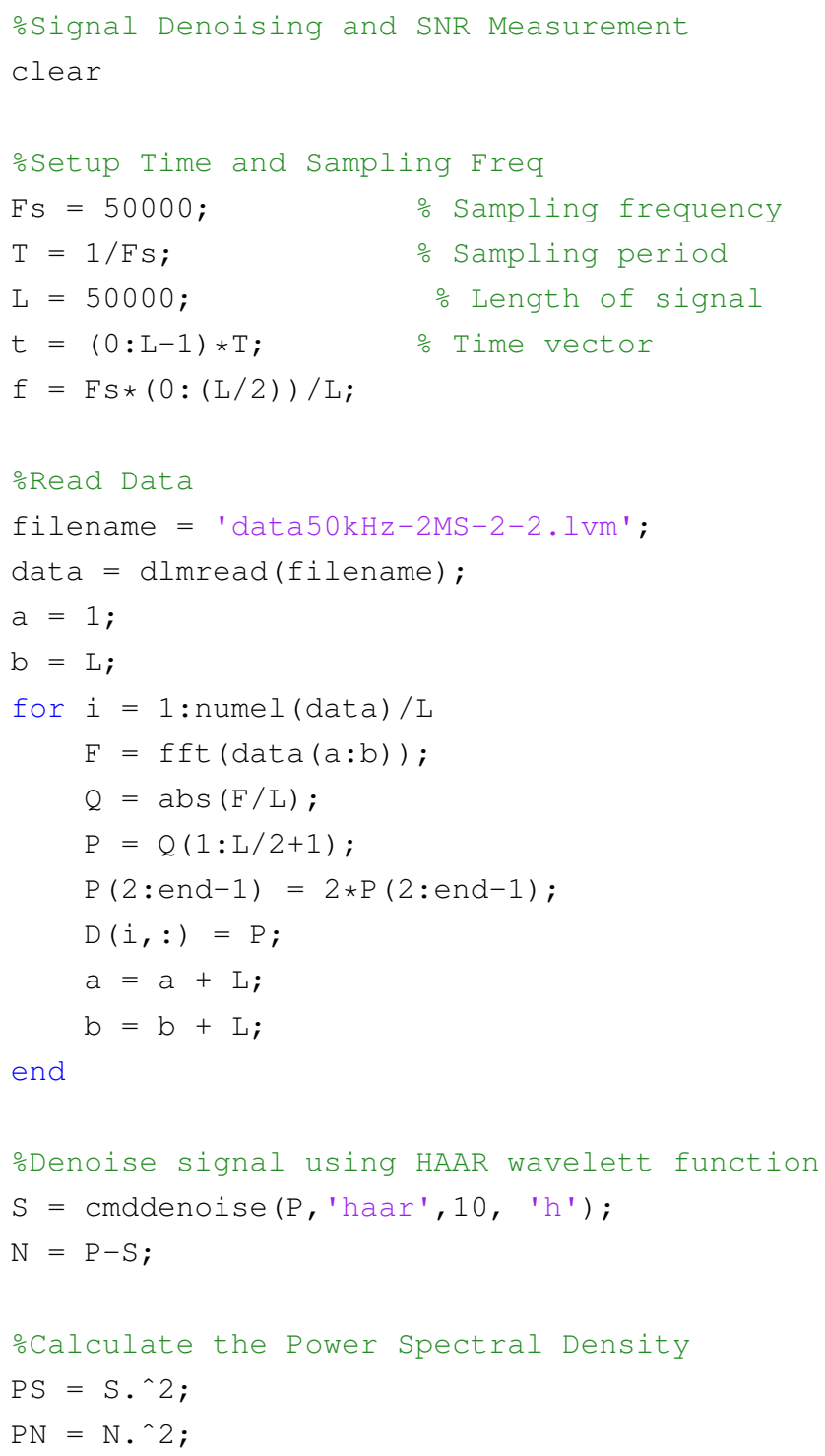




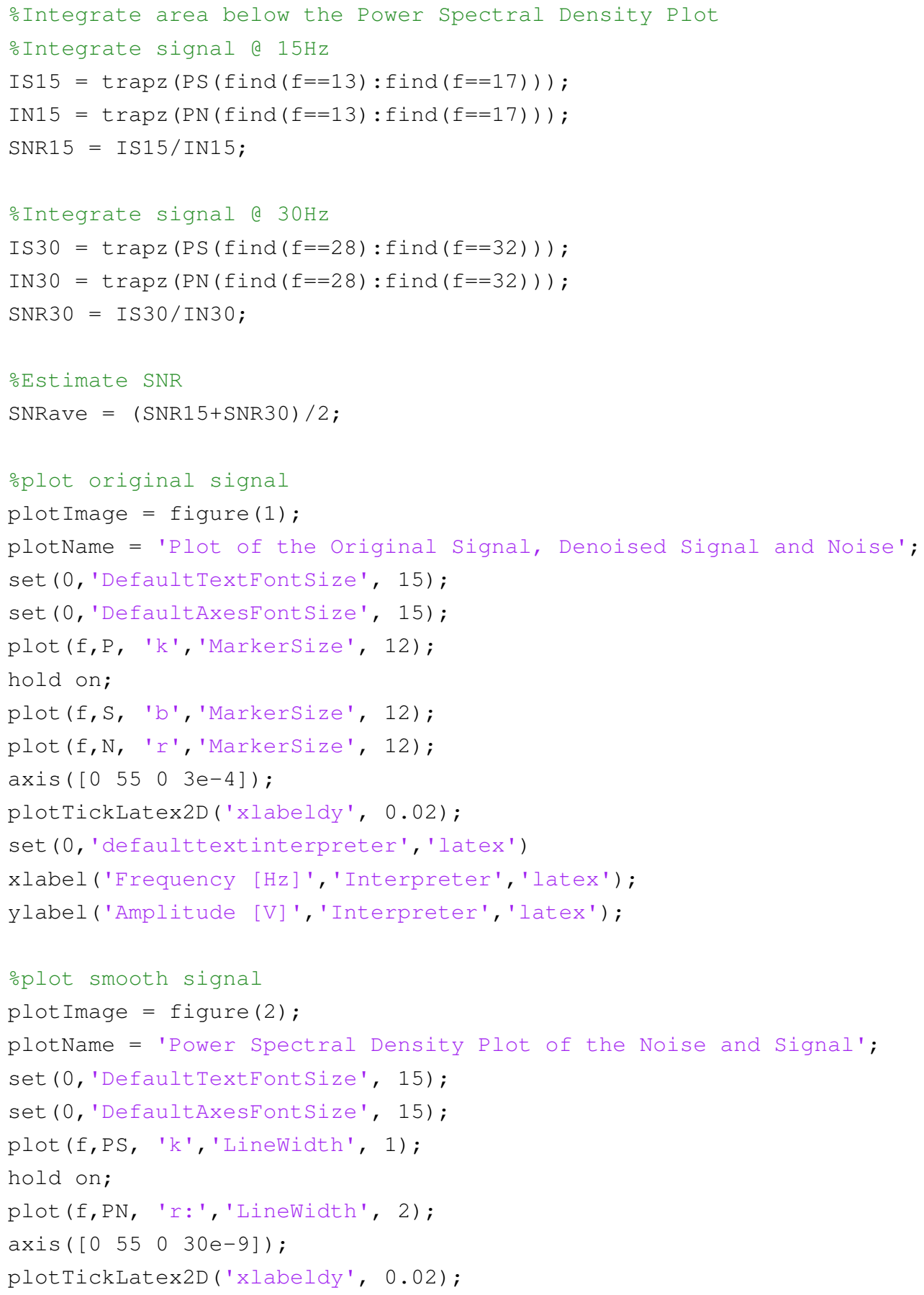




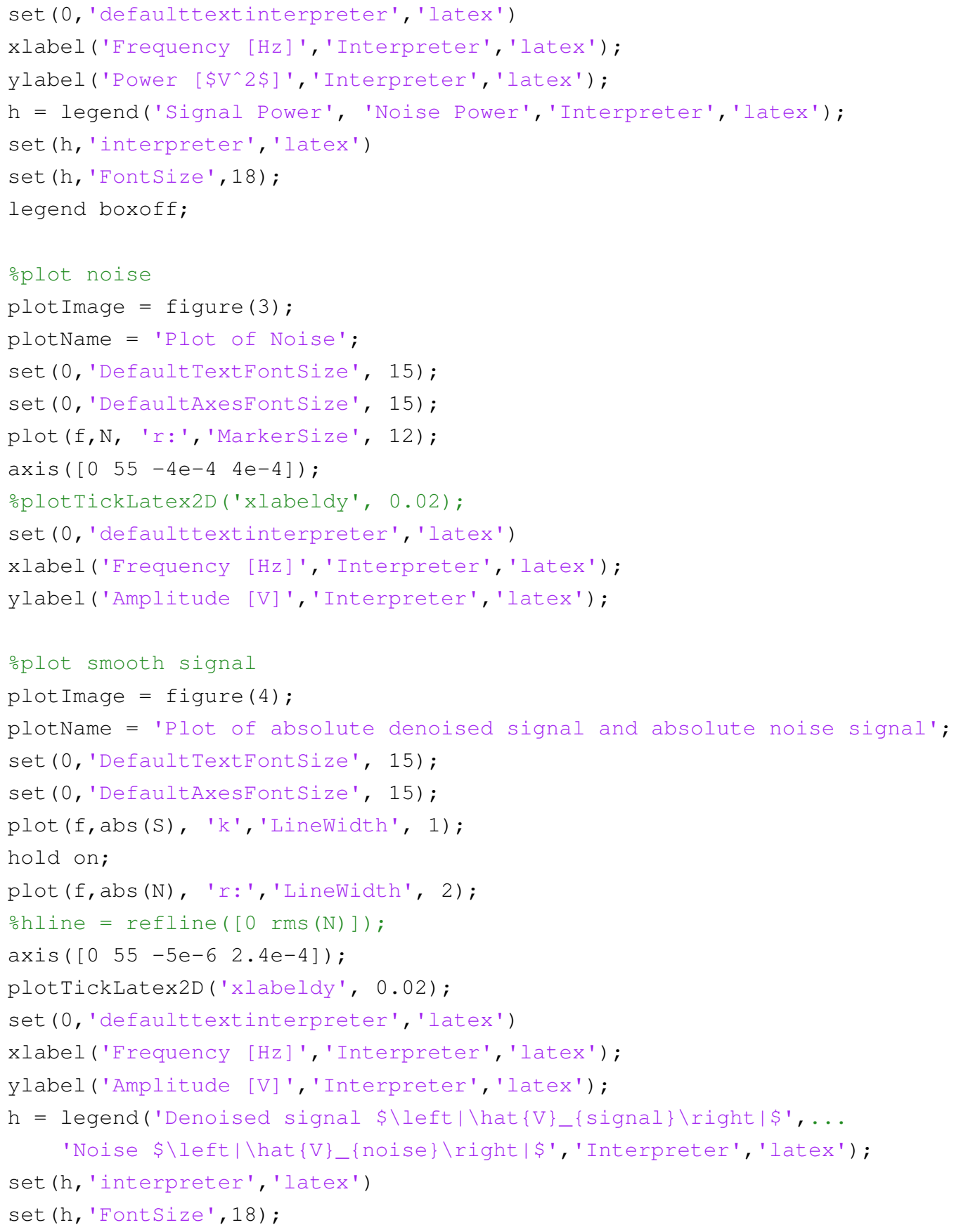




\section{B.3 Scatter Plot Generation and Calibration Curve}

The MATLAB code to generate the scatter plot and the calibration curve is presented below.

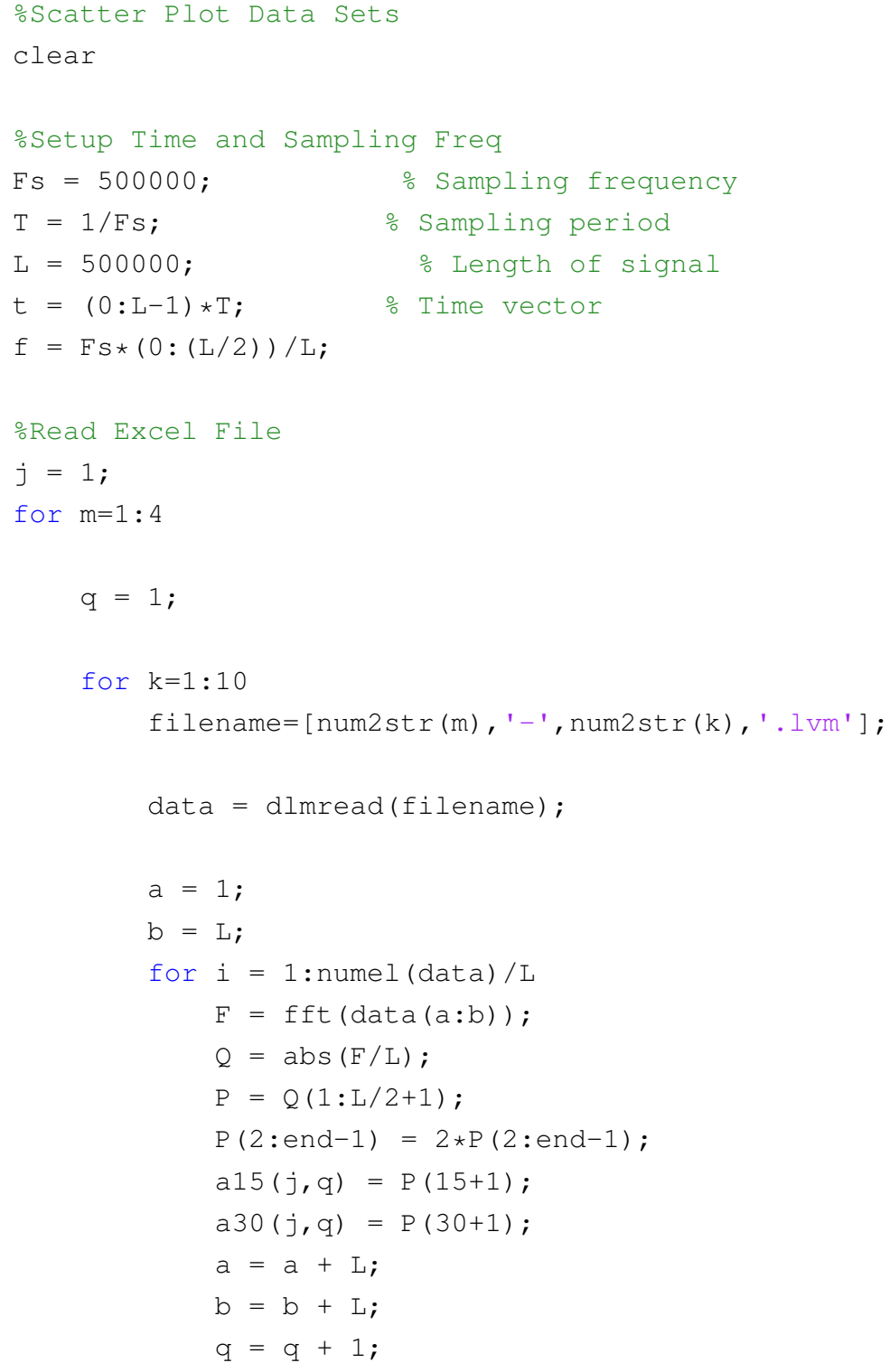




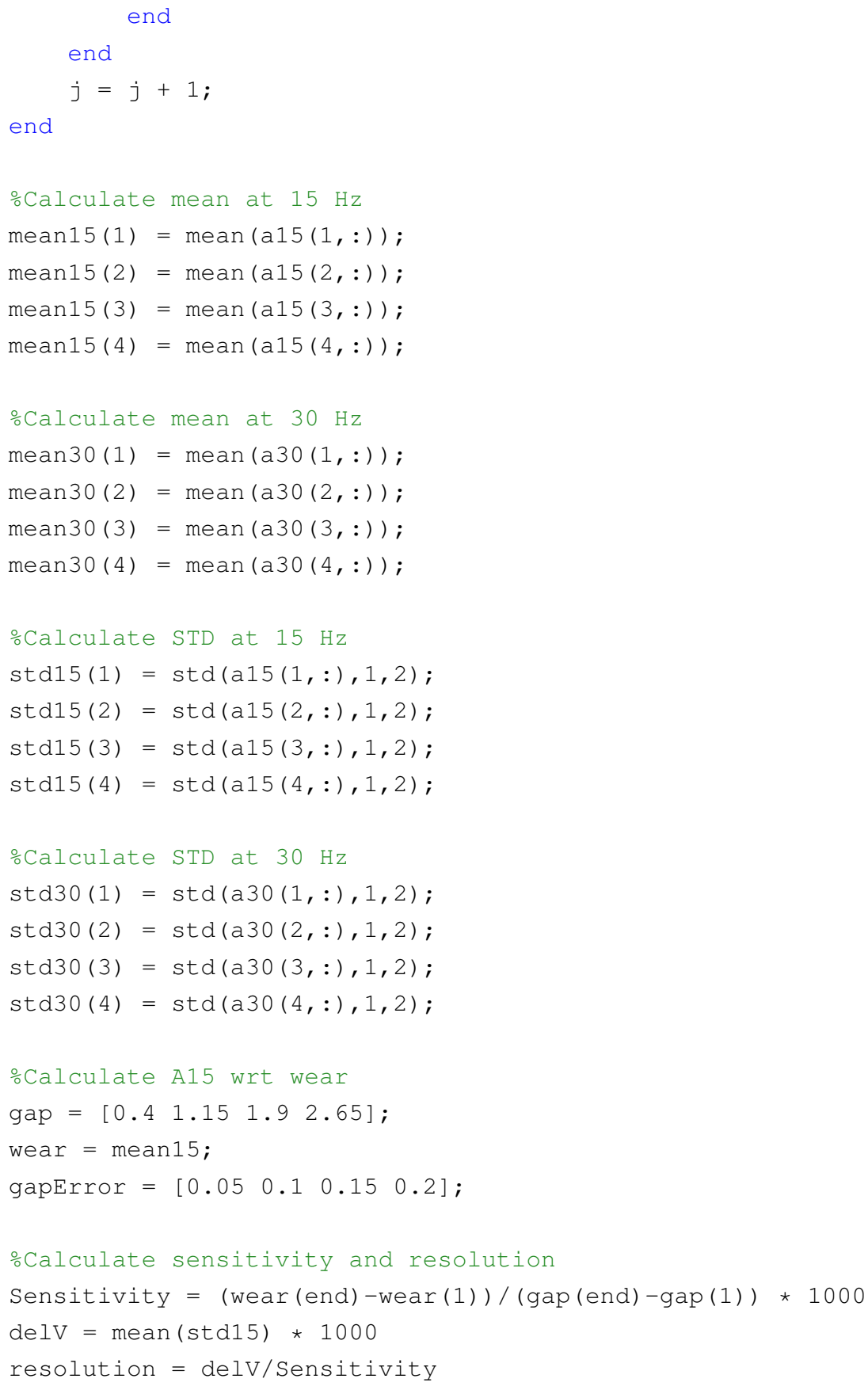




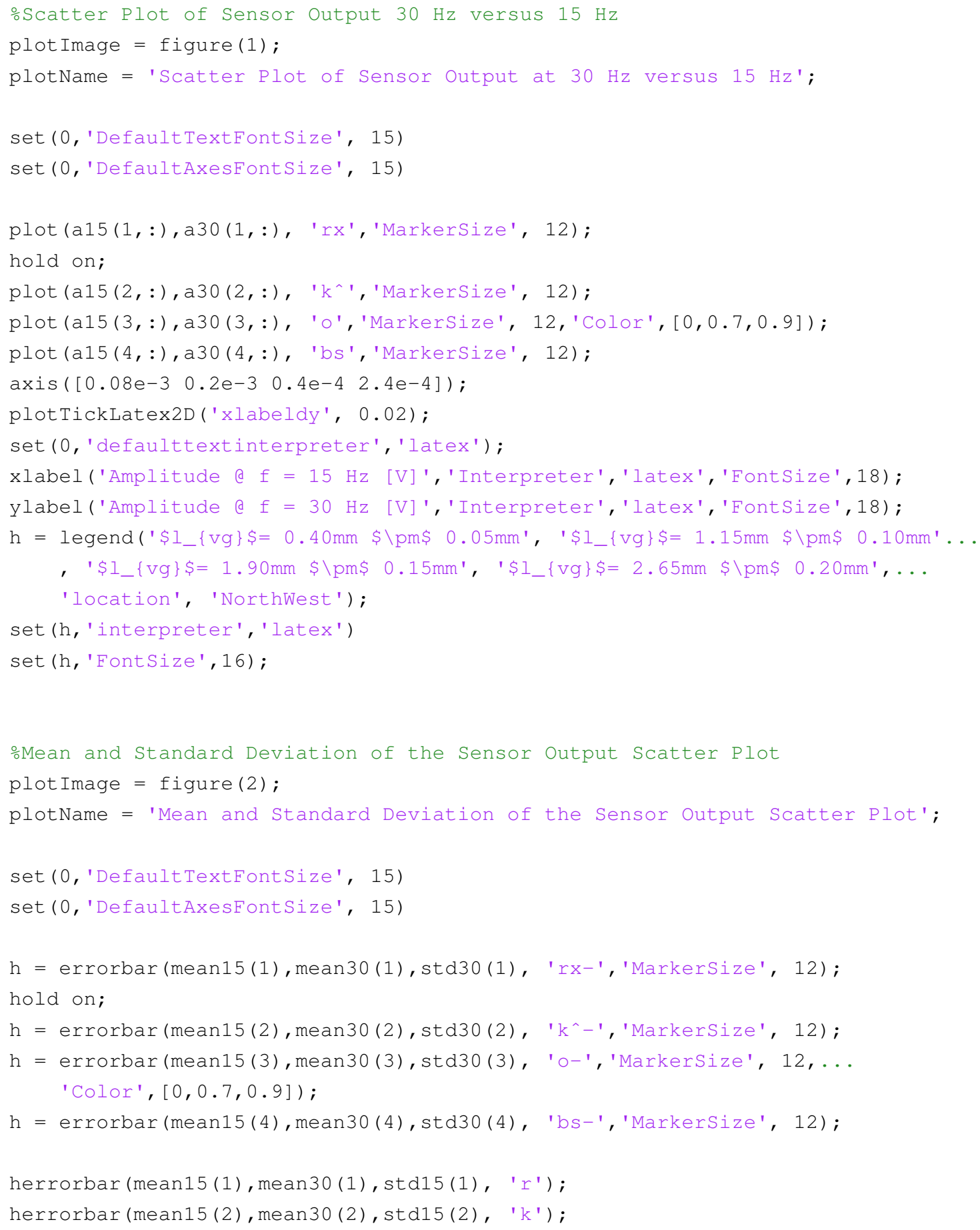




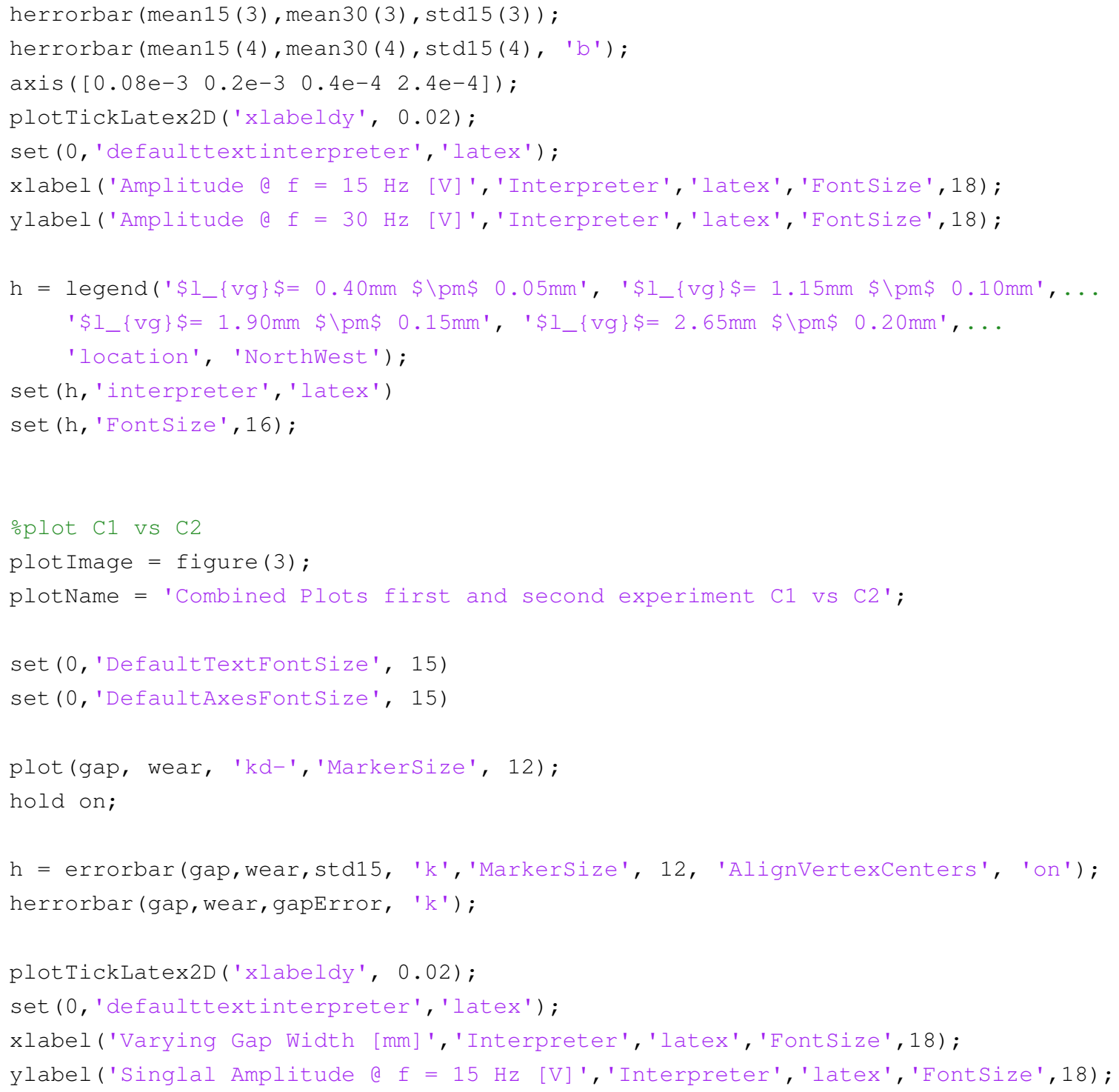




\section{Appendix C}

\section{Sensor Optimization}

To improve the quality of the sensor's output signal, several methods are used as described in Chapter 3. However, many of the methods used did not significantly affect the signal quality. This section will go into the detail of the cases not mentioned in the body of the thesis.

\section{C.1 Permanent Magnet Geometry Optimization}

To select the best suitable sensing technique, three different techniques were considered as described in Section 2.2. To ensure that each method is optimized to derive the best performance possible in the comparison, the geometry of the permanent magnet in the permanent magnet model sensor needs to be optimized. Since this method is not used for the sensor, the derivation of the technical calculation is only included in the Appendix section of the thesis. To optimize the geometry, the ratio between the magnet's length and cross-sectional area

$$
g=\frac{l_{\text {magnet }}}{A_{\text {magnet }}}
$$

is considered as a single parameter. The slope of the load-line equation of the magnet 


$$
m_{l}=\frac{B_{m}}{H_{m}}=-\frac{g}{\frac{l_{v g}}{\mu_{0} A_{v g}}+\Re_{\text {const }}}
$$

is also written as a ratio between the operating flux density $B_{m}$ and the operating field intensity $H_{m}$. By combining the equation for the demagnetization curve of the permanent magnet as shown in Equation 2.15 and the magnet's load-line equation, the operating magnetic flux density

$$
B_{m}=\frac{m_{l} H_{c} B_{r}}{m_{l} H_{c}-B_{r}}
$$

can be written in terms of the slope of the load-line equation $m_{l}$, magnet's remanent flux density $B_{r}$, and the magnet's coercivity $H_{c}$. To maximize the change in the operating flux density as the reluctance of the magnetic circuit varies from the initial reluctance $\Re_{i}$ to the final reluctance $\mathfrak{R}_{f}$, the change in the flux density

$$
\Delta B_{m}=B_{m\left(@ l_{v g}=0.65 \mathrm{~mm}\right)}-B_{m\left(@ l_{v g}=2.50 \mathrm{~mm}\right)}
$$

is calculated as the varying gap $l_{v g}$ increases. By solving this equation for the geometric ratio of the permanent magnet $g$ in the load-line equation, the ratio

$$
g=\mu \sqrt{\Re_{i} \Re_{f}}
$$

can be written in terms of the initial and final reluctance values as stated in Equation 2.19

\section{C.2 Signal Quality Improvement}

As mentioned in Section 3.4, to calculate the peak amplitudes used for the scatter plot as shown in Figure 3.27 four different techniques are used. First method is to simply take the amplitude voltage at $15 \mathrm{~Hz}$ and $30 \mathrm{~Hz}$ and plot them against each other. To potentially improve the quality of the scatter plot, the ratio of the peak amplitude can also be calculated with respect to the amplitude of the FFT plot at $70 \mathrm{~Hz}$ which is the sensor's excitation frequency. Figure C.1 shows the resulting scatter plot. As it can be seen, the resulting plot is not much improved from the 
original plot.

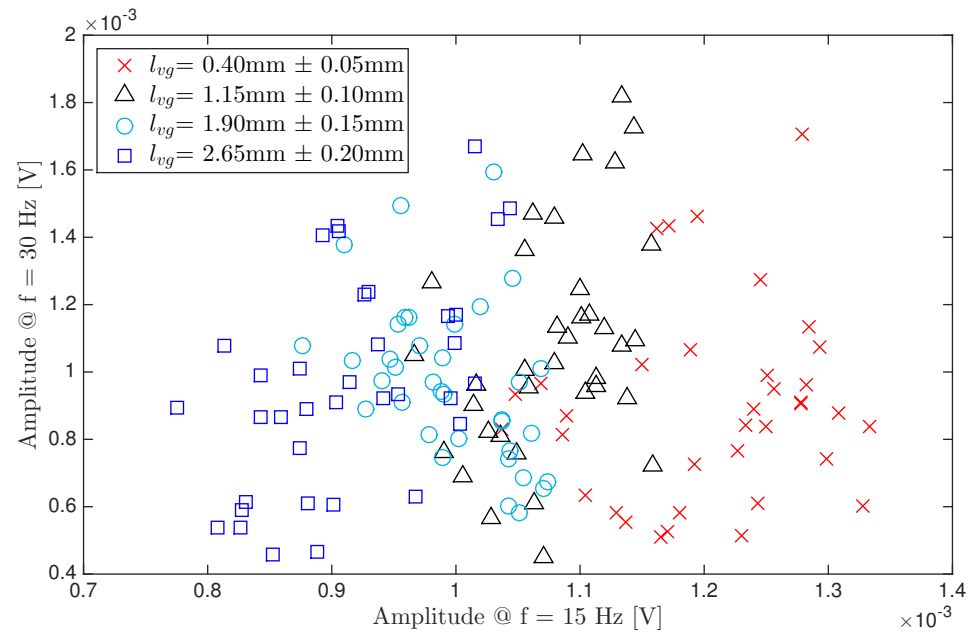

Figure C.1: Scatter plot of the ratio of signal at $30 \mathrm{~Hz}$ over $70 \mathrm{~Hz}$ versus the signal ratio of $15 \mathrm{~Hz}$ over $70 \mathrm{~Hz}$ at various gap widths.

Another method to obtain the scatter plot is to evaluate the ratio of the peak amplitude at both frequencies with the RMS value of the signal noise at each measurement point. Figure C.2 represents the scatter plot referenced to this technique. 


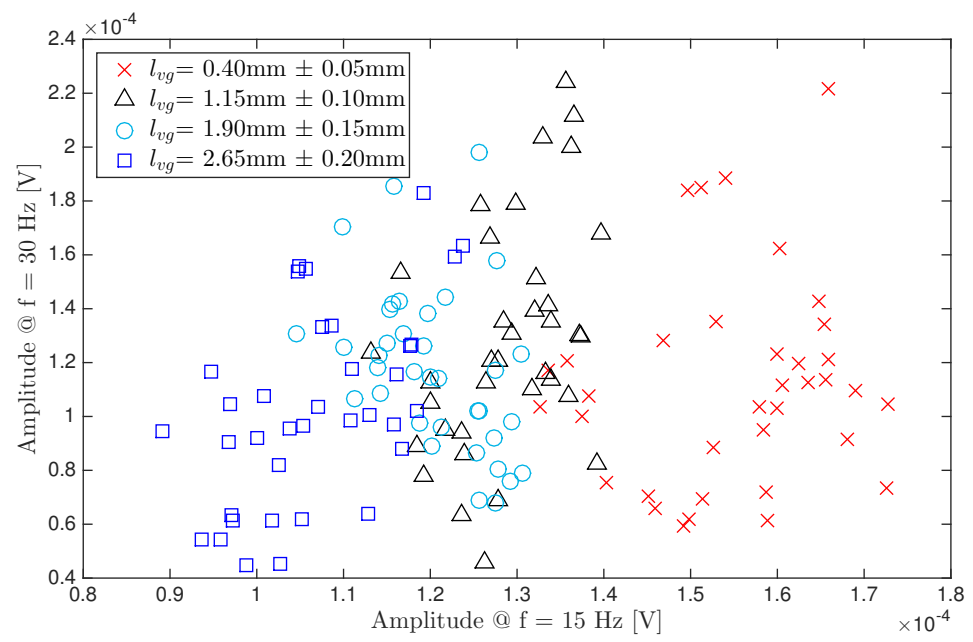

Figure C.2: Scatter plot of the ratio of signal at $30 \mathrm{~Hz}$ and $15 \mathrm{~Hz}$ over the RMS value of noise.

Lastly, the scatter plot can also be derived form the difference between the original signal and the noise calculated through the means of wavelet denoising function in MATLAB. Figure C.3 represents the scatter plot of the peak amplitudes at $15 \mathrm{~Hz}$ and at $30 \mathrm{~Hz}$ for the denoised signal. 


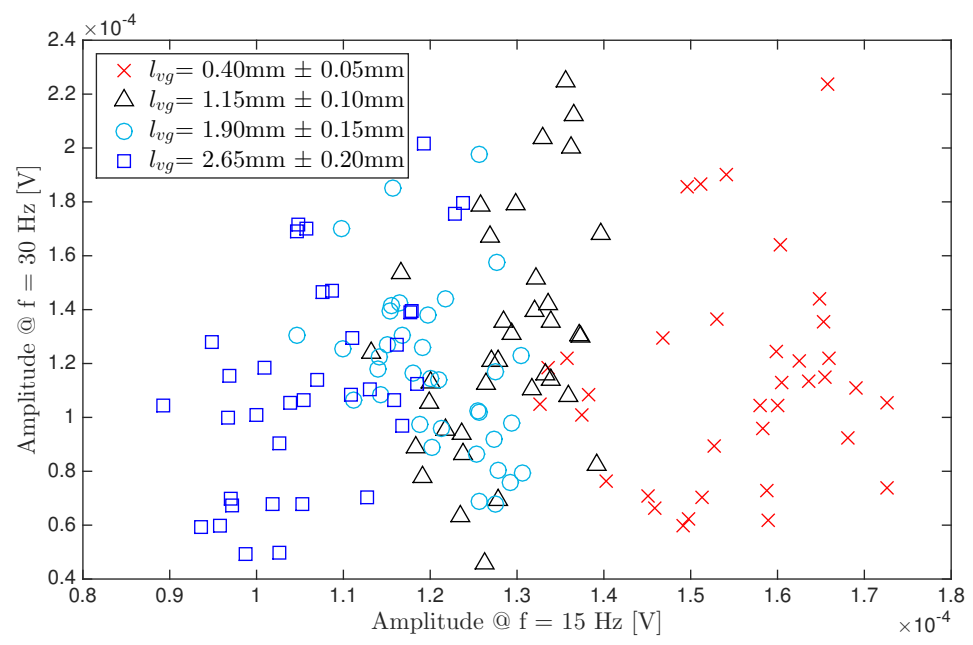

Figure C.3: Scatter plot of the denoised amplitude at $30 \mathrm{~Hz}$ versus the denoised amplitude at $15 \mathrm{~Hz}$ at various gap widths. 